\title{
STRATEGIES AND PROCESSES EMERGENCY DEPARTMENT NURSES CONSIDER IMPORTANT TO SAFELY MANAGE DURING AN INFLUENZA PANDEMIC: A QUALITATIVE DESCRIPTIVE STUDY
}

By

Jessica Colleen Mary Lockett

\begin{abstract}
A thesis
submitted to the Victoria University of Wellington

in partial fulfilment of the requirements for Master of Nursing Science
\end{abstract}

Victoria University of Wellington

2020 


\begin{abstract}
The influenza virus is responsible for significant morbidity and mortality worldwide each year, with influenza pandemics occurring every 10 to 50 years and responsible for millions of deaths and substantial economic impact. Increasing globalisation through travel and trade means New Zealand is vulnerable to the risks of pandemic influenza, placing a strain on the healthcare system, putting lives at risk and posing a significant cost to the country. Emergency Departments are at the frontline of New Zealand's healthcare system and are a crucial component in the response to an influenza pandemic, however little research has been done on the impact such an event would have on the nursing staff who work in this area and the care they provide to influenza patients.
\end{abstract}

This study aims to explore what New Zealand Emergency Department nurses perceive as the biggest challenges to nursing care and staff safety during an influenza pandemic, in order to provide information on how to ensure the engagement of these nurses at the frontline of the pandemic response. With a lack of evidence-based research available, a qualitative descriptive design was used to allow an exploration of the first-hand perspectives of Emergency Department nurses, gaining meaningful insights into a phenomena which has been little explored.

Sixteen nurses from two Emergency Departments participated in face-to-face interviews conducted using semi-structured questions. Raw data was transcribed, and an inductive approach was taken to data analysis, guided by the principles of both content and thematic analysis.

The findings demonstrate that Emergency Department nurses work in an environment that poses risk to patient and staff safety every day, and an understanding of these safety problems is provided in the theme 'the everyday reality for Emergency Department nurses'. Working within this context shapes the 
fears that Emergency Department nurses hold about what could happen if an influenza pandemic were to affect New Zealand in the future, and are summarised within the theme 'fears for a pandemic'. The final theme, 'strategy and planning for pandemics' provides insight into how Emergency Department nurses feel these issues could be managed within future pandemic planning at Emergency Department, District Health Board and government level.

This thesis identifies both existing and potential future safety concerns in relation to the management of influenza in New Zealand Emergency Departments, affecting the safety of patients and staff. It also provides specific multi-level and multiagency recommendations for future pandemic plans that could help to mitigate the significant risks highlighted by those who work within the system every day.

Key words: Infectious Disease, Disease Outbreaks, Occupational Safety, Strategic Planning 


\section{ACKNOWLEDGEMENTS}

I wish to thank all the people who have helped me on my thesis journey, to whom I am truly grateful and forever indebted.

Firstly, to my supervisors: Dr Caz Hales and Dr Kathy Nelson. I wish to express my sincere gratitude for all of the guidance and encouragement you have given me. The immense knowledge and passion you both bring to research has been inspirational, and I am profoundly grateful for my luck in having you supervising my work.

To my fiancé Steve Lockett, thank you for your love and support. Without it, I would not have gotten through this thesis, buying our first home and planning our wedding! Thanks for putting up with me when I was stressed, believing in me and encouraging me to get to the finish line. Now let's get married!

To my Mum, Phillipa Buckley, thank you for helping with wedding planning, allowing me more time to finish my work. To my Dad, Mike Buckley, thank you for being a second pair of eyes.

To my friends, thank you for putting up with my frequent rainchecks on socialising because "I have to do my thesis", I'm finally free! A special thank you also to Victoria for being my thesis guru and encouraging me to the end.

Finally, thank you to the ED nurses who participated in my research, without whom none of this would have been possible. Thank you for trusting me with your thoughts, feelings and experiences, I truly value your co-operation and insight. It's tough being an ED nurse sometimes, hopefully your contributions will help me to make things just a little bit easier for us all. 


\section{TABLE OF CONTENTS}

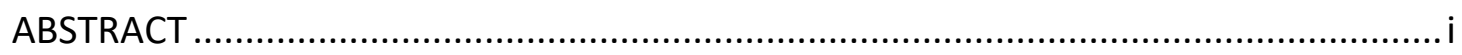

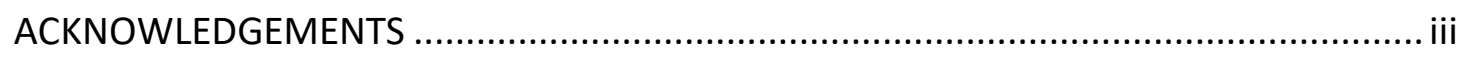

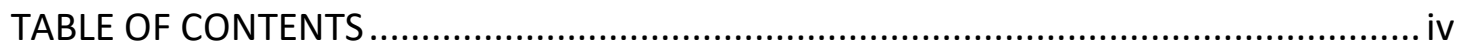

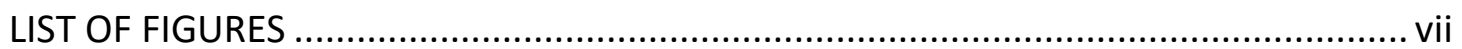

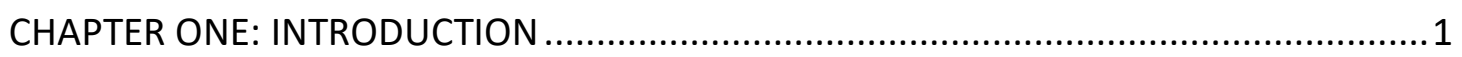

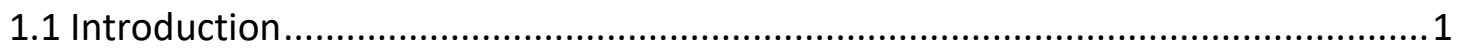

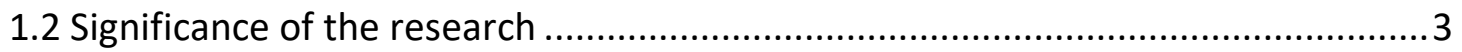

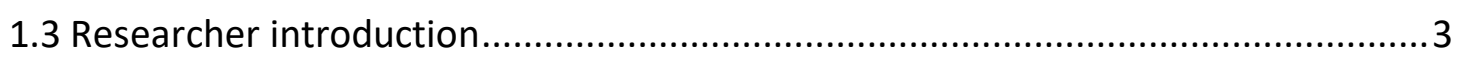

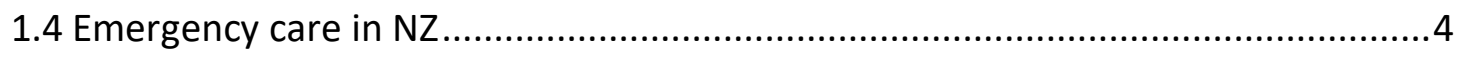

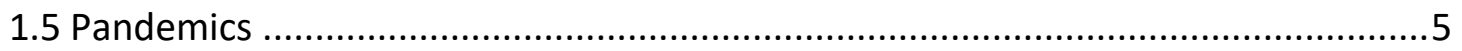

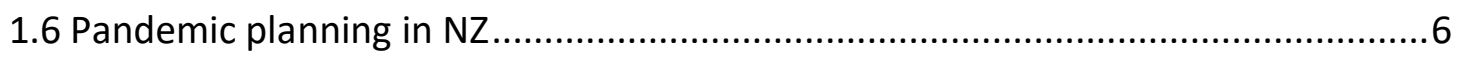

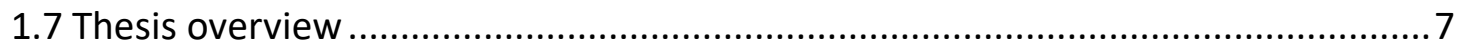

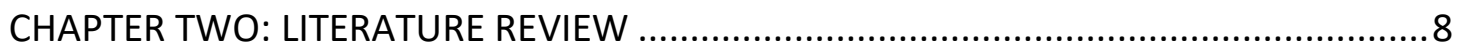

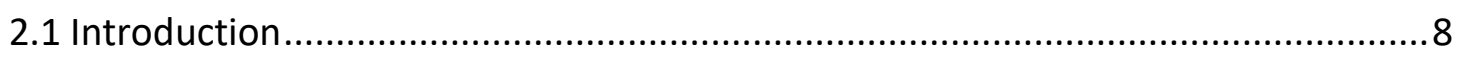

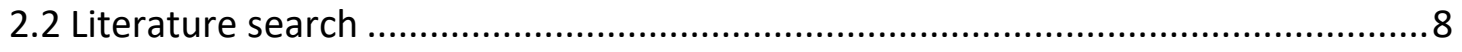

2.3 Occupational exposure to infectious disease in pandemic scenarios ....................9

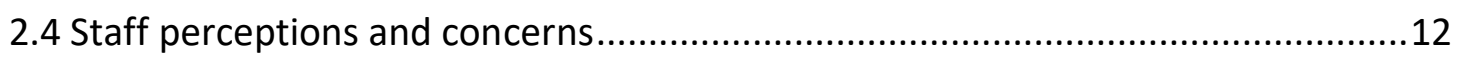

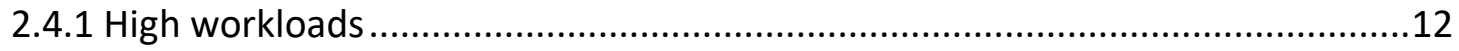

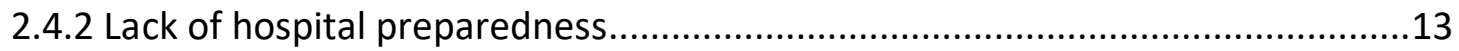

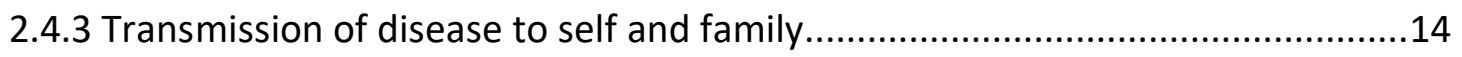

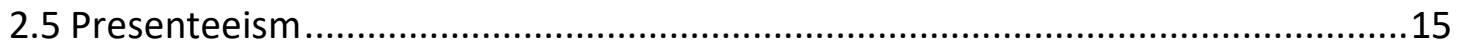

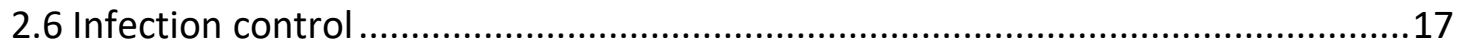

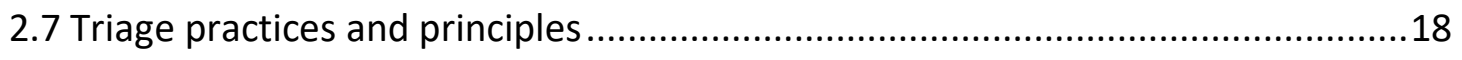

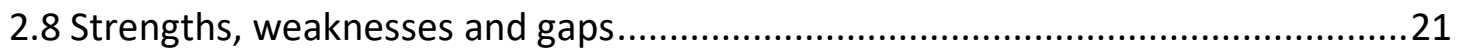

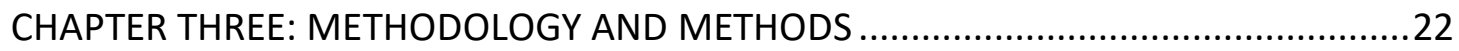

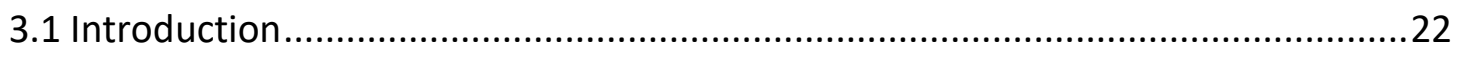

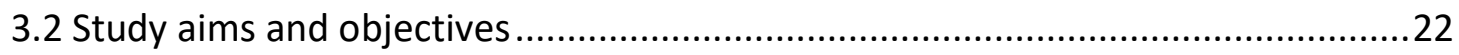

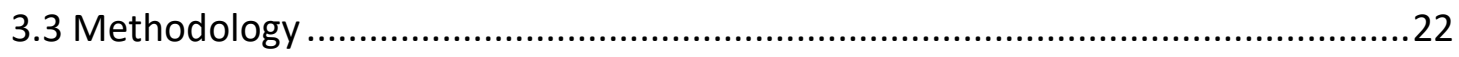

3.3.1 Descriptive, exploratory and interpretive designs .........................................23 


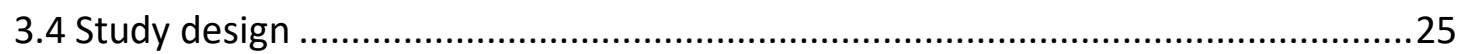

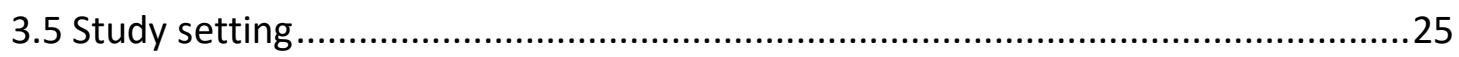

3.6 Participant selection, recruitment and sampling strategy .................................25

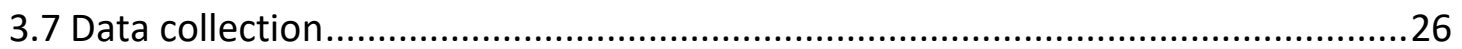

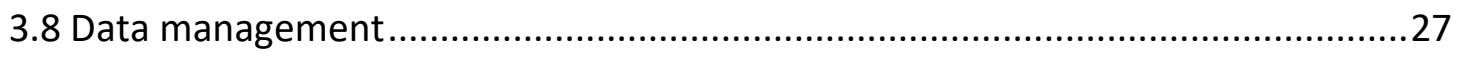

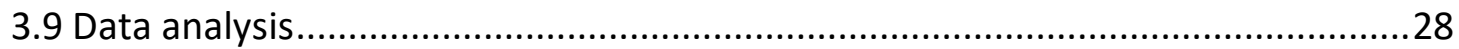

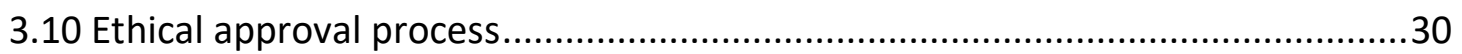

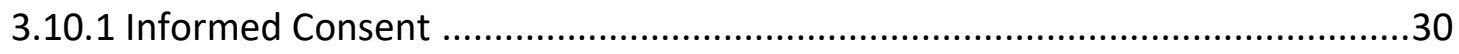

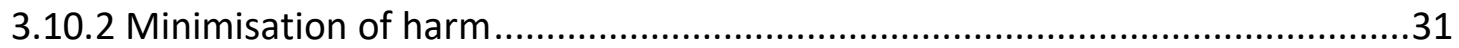

3.11 Treaty of Waitangi and respect for cultural diversity ........................................31

3.12 Protecting the identities of the study participants ..........................................32

3.13 Rigour and trustworthiness of the research process ........................................32

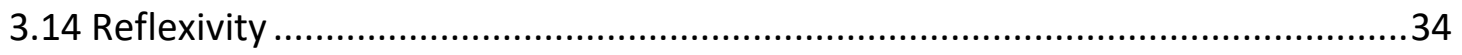

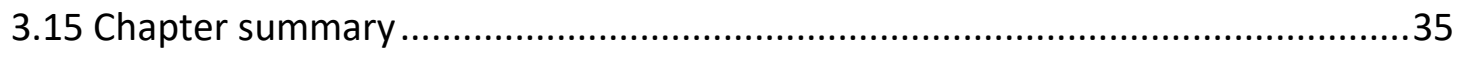

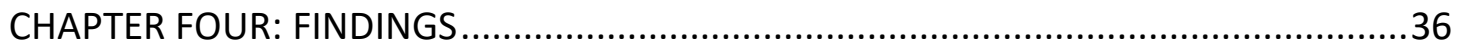

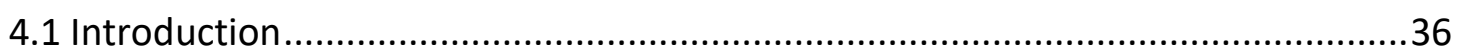

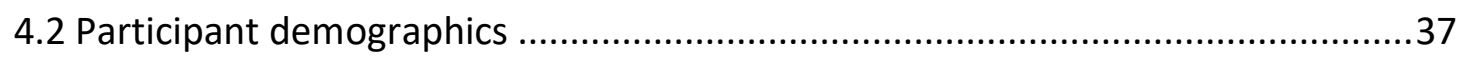

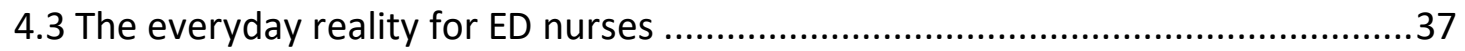

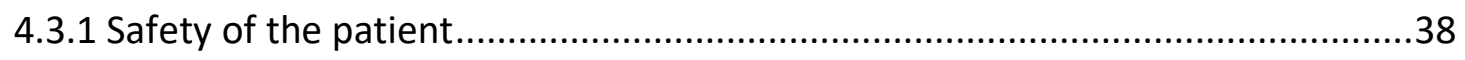

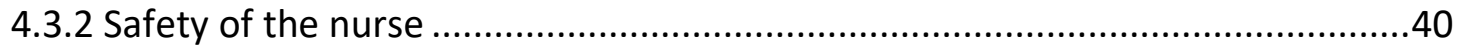

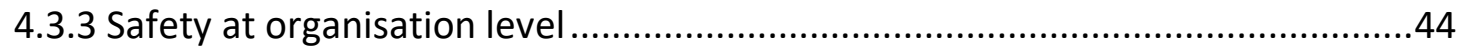

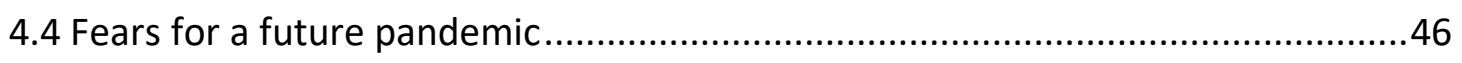

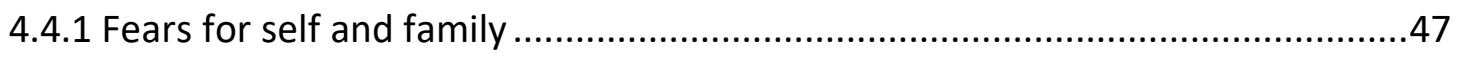

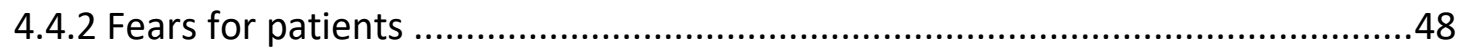

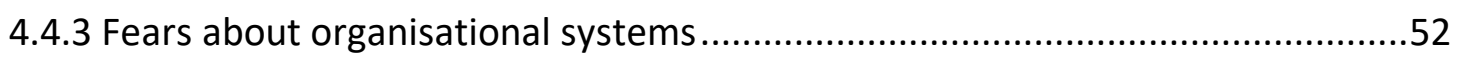

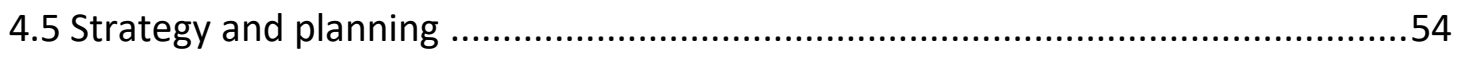

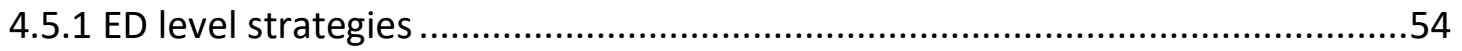

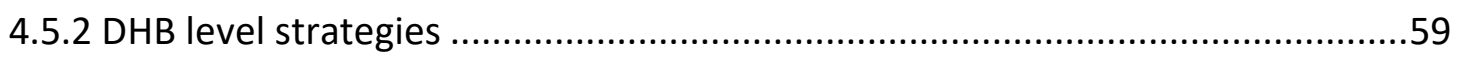

4.5.3 Government level strategies .....................................................................61

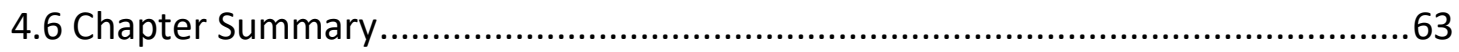

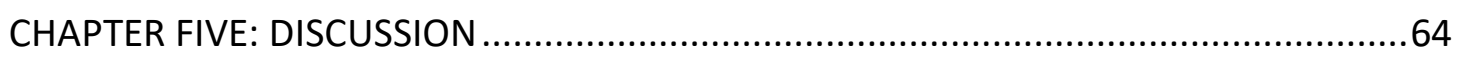

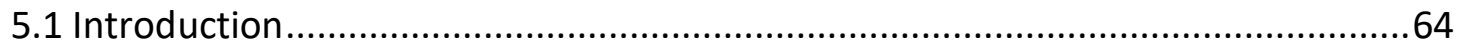


5.2 The everyday reality for ED nurses

5.2.1 Safety problems through nurse actions ....................................................65

5.2.2 Safety problems through nurse omission ...................................................68

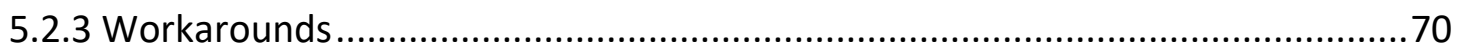

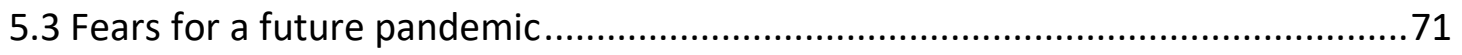

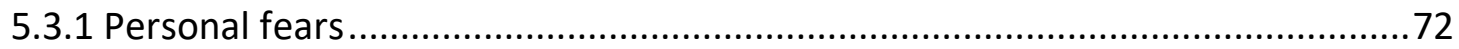

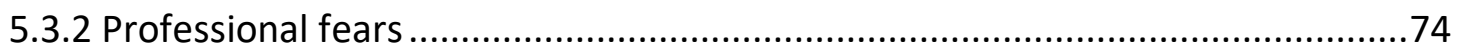

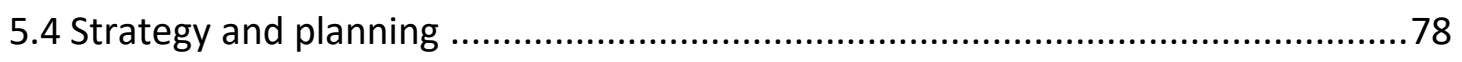

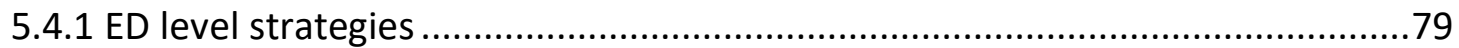

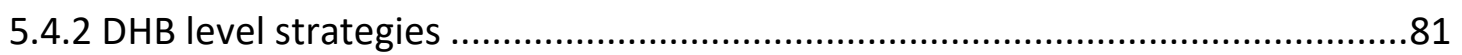

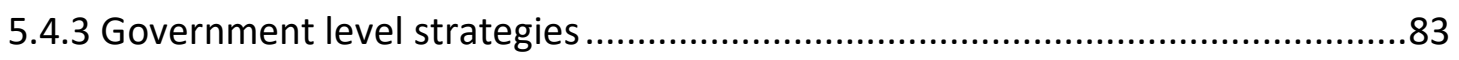

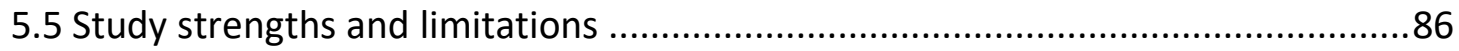

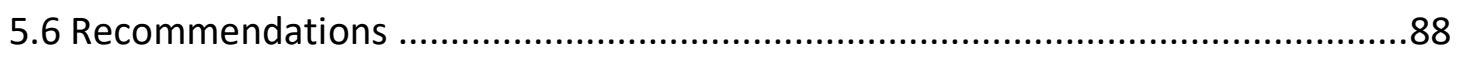

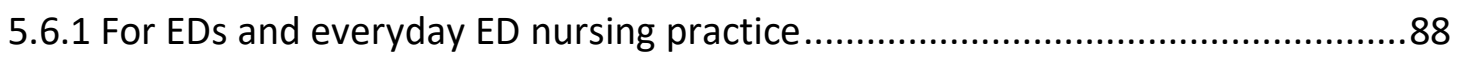

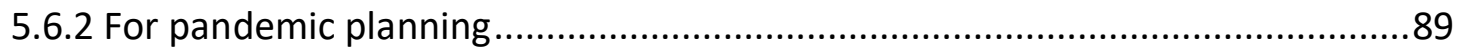

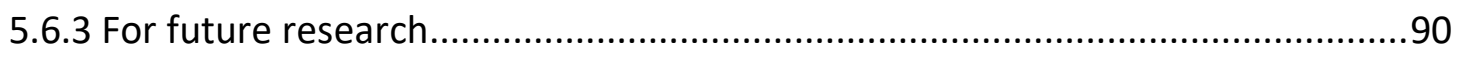

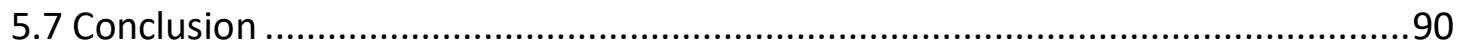

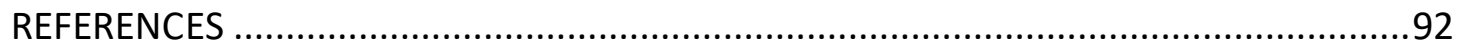

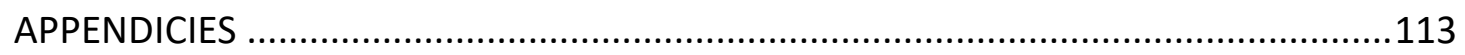

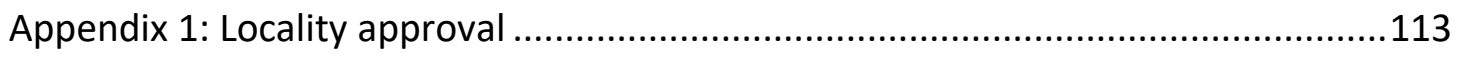

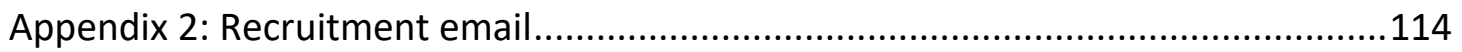

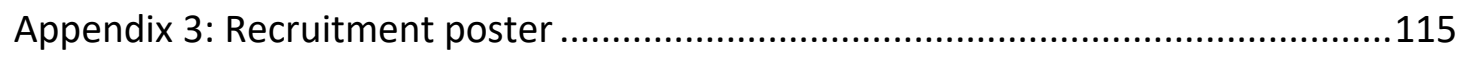

Appendix 4: Participant demographic questionnaire …...........................................116

Appendix 5: Semi-structured interview guide ...................................................117

Appendix 6: Example of NVivo 12 content code (marked by inverted commas) ......119

Appendix 7: Example of NVivo 12 thematic codes and raw data ............................120

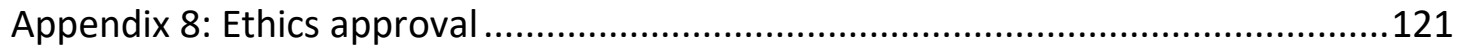

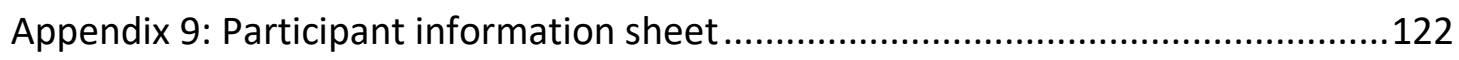

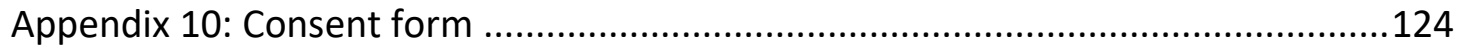




\section{LIST OF FIGURES}

Figure 1: Screening process of retrieved articles, including the stages of excluded articles and the assessment of eligibility

Figure 2: ED nurses' perspectives on existing and future issues regarding pandemic planning in NZ ... .36 


\section{CHAPTER ONE: INTRODUCTION}

\subsection{Introduction}

The Emergency Department [ED] is a dynamic and fast-paced environment where patients of all ages receive urgent care for a range of conditions, including major injuries and critical illnesses. Patients present with undifferentiated signs and symptoms, and it is the role of the ED team to stabilise, diagnose and treat these conditions before discharging them home or referring patients for ongoing care (Australasian College for Emergency Medicine [ACEM], 2019). The undifferentiated nature of presentations to ED means that patients with infectious diseases may not be identified immediately on arrival, placing other patients as well as ED staff at risk for transmission of disease (Fusco \& Puro, 2012). This is especially significant during an infectious disease pandemic situation, where a previously unknown virus that humans have little or no resistance to spreads across the world, causing high rates of illness and death (Dicker, Coronado, Koo \& Gibson Parrish, 2012). Whilst geographically isolated, New Zealand [NZ] is at risk from a pandemic due to increased travel and trade connections internationally (Ministry of Health [MOH], 2017). The influenza virus is considered the most likely cause of the next worldwide pandemic, due to the ease of disease transmission and frequently evolving nature of the virus (World Health Organisation [WHO], 2014)

With NZ EDs already struggling to manage overcrowding, an influenza pandemic would cause a surge of presentations, potentially overwhelming healthcare infrastructure (MOH, 2017). Although EDs are a crucial part of the NZ health system, there is minimal infromation included in the government pandemic plan for EDs specifically. The staff who work within the ED environment everyday are best placed to identify the actual and potential problems that could occur during an influenza pandemic. This thesis sought to determine what strategies and processes ED nurses consider important to safely manage during an influenza pandemic. Furthermore, it aimed to identify ED nurses' perspectives around the provision of nursing care during 
a pandemic, and their perspectives around the safety of themselves, their families and patients.

The term pandemic refers to a disease that has spread over a wide geographical area, crossing international borders and affecting a large number of people (Dicker et al., 2012). The last worldwide pandemic occured in 2009, when the H1N1 influenza virus was responsible for infecting between 11 and 21 percent of the global population and estimated to have caused up to half a million deaths and a rapid increase in demand for ED services worldwide (Centers for Disease Control and Prevention [CDC], 2019). Prior to this, an outbreak of severe acute respiratory syndrome (SARS) occurred, affecting 26 countries between 2002 and 2003 and causing over 8000 infections and 774 deaths. Twenty one percent of all SARS cases occurred in healthcare workers (WHO, 2003).

Within the past year, two outbreaks of infectious diseases have occurred causing widespread illness and deaths. An epidemic of measles, the highly contagious airborne virus, was declared in Samoa in 2019. An epidemic refers to the sudden increase of a disease in excess of the normal expected case numbers in a specific population and can develop into a pandemic if the disease spreads to surrounding regions (Dicker et al., 2012). Measles has affected thousands in Samoa and has killed at least 70 individuals, mainly children (WHO, 2019). Most recently, a new strain of coronavirus (2019-nCov) emerged in Wuhan, China. This virus comes from the same family as SARS and causes severe pneumonia and death in some individuals. The WHO estimates the basic reproductive number, or average number of new cases each positive case will generate, of this coronavirus to be approximately 2.5. This is comparative to SARS (approximately 3), and higher than 2009 H1N1 influenza (1.4-1.6) (Coburn, Wagner \& Blower, 2009; WHO, 2003, 2020). The virus has since spread to other countries in Asia and elsewhere, including Australia and the United States. There are currently no reported cases in NZ, but the $\mathrm{MOH}$ is monitoring the situation as it evolves $(\mathrm{MOH}$, 2020). 


\subsection{Significance of the research}

There is no NZ-based research on the perspectives of ED nurses regarding the provision of nursing care during a pandemic, or the strategies they consider necessary to manage during such a scenario. Conducting NZ-based research is important as the findings will be specific to NZ's unique healthcare system. Undertaking this research now will provide guidance to EDs, $\mathrm{DHBs}$ and the $\mathrm{MOH}$ and allow these services to update their plans in advance of a pandemic occurring in the future.

The choice was made to focus this research within the ED context because ED is the initial access point for healthcare for much of NZ's population and would therefore bear a heavy burden during an influenza pandemic. Despite this, there is limited information within regional and national pandemic plans on how EDs should manage the expected increase in patient presentations. Furthermore, although many different members of the healthcare team work within ED, this research is limited to investigating the concerns of nursing staff specifically. This decision was made as nurses make up the largest staff cohort within the ED, are responsible for the initial assessment and treatment of most ED patients, and have the most patient contact, increasing their risk of being exposed to influenza.

\subsection{Researcher introduction}

As an ED nurse with over a decade of experience, I have witnessed firsthand each winter the impact seasonal influenza has upon EDs. Influenza is difficult to identfy due to the sometimes atypical presentation pattern, and is challenging to treat, as options are mainly limited to supportive care. The disease affects patients of all ages and can cause critical illness and death. Each year, the management of these infectious patients challenges the resourcefulness of the hospital, as isolation rooms are limited and hospital occupancy often over capacity. My first year of nursing as a new graduate coincided with the H1N1 influenza pandemic of 2009, and although this pandemic was less severe than previous influenza pandemics, overall management appeared chaotic. Many of my colleagues felt more should have been done to isolate infectious patients and help limit the transmission of disease to other patients and ED staff. These factors 
sparked my interest in the topic of ED pandemic preparedness, and made me curious about the gaps ED nurses feel exist in current pandemic planning and what more could be done to manage a pandemic at the hospital frontline.

\subsection{Emergency care in NZ}

To understand the context of this research, it is crucial that the delivery of emergency care in NZ is understood. NZ operates a universal, publicly-funded healthcare system for its almost 4.9 million citizens, with responsibility divided amongst 20 DHBs to provide healthcare to their regional populations (Rees, 2014; Statistics New Zealand, 2019). The DHBs' role is to improve and promote the health of their populations through the provision of community-based and hospital-based services ( $\mathrm{MOH}, 2013)$. EDs provide emergency acute care and manage fluctuating numbers of patients with a wide variety of illnesses and injuries ranging from minor to critical. EDs are often the first point of call for patients seeking healthcare, and there is signifcant demand for ED services in NZ, with presentations increasing by 10 percent in the five years between 2011 to 2015 (ACEM, 2019). Patients are assessed by a trained triage nurse to determine how urgently a patient can wait to see a doctor and recieve treatment for their condition. Triage is the process of sorting patients into categories that determine the maximum clinically-appropriate time each patient can wait for timecritical emergency interventions and care. NZ EDs use the Australasian Triage Scale to sort patients into five categories, from category one (immediately life-threatening), through to category five (less urgent or minor conditions) (ACEM, 2016).

With presentation rates increasing faster than population growth, EDs worldwide are experiencing significant issues with overcrowding and access block (Ardagh \& Drew, 2015). Access block is a term used to describe the delay in the admission of patients from ED to inpatient areas within a hospital. It has been estimated that up to 40 percent of ED staff time is used caring for patients who are waiting in the ED for an inpatient bed, rather than caring for newly arrived emergency patients (Forero, McCarthy \& Hillman, 2011). Ovecrowding describes the situation where the number of patients in ED exceeds the available physical or staffing capacity, negatively 
impacting on ED function (Forero, McCarthy \& Hillman, 2011). Access block is an important contributing factor behind ED overcrowding, and both of these situations have proven links to decreased efficiency, increased hospital length of stay and increased adverse events and mortality rates (ACEM, 2019; Zhou et al., 2012). Managing these situations remains a constant and complex challenge for those working within the ED system, and wider hospital and healthcare services.

\subsection{Pandemics}

The likelihood of a widespread outbreak of infectious disease has grown significantly with increased global travel and trade links, large-scale migration and population urbanisation (Madhav et al., 2017). The spread of disease is ubiquitous amongst populations and is difficult to avoid when living closely amongst others. In most cases, the burden of disease within a population is borne by the very young and the very old, and those with chronic conditions (WHO, 2010). Some diseases learn to evolve and adapt to overcome the human immune response, and it is these diseases that pose a risk for developing into an epidemic or pandemic.

Influenza is considered by the WHO to be the most likely pathogen to cause the next global pandemic because it can also infect animals, making it a zoonotic virus. The presence of the disease in animal populations provides the virus more ability to mutate into a new viral strain that humans have little or no immunity against (WHO, 2018). Each year, there is an estimated one percent probability that an influenza pandemic will occur and result in up to six million deaths (Madhav et al., 2017). Pandemics not only carry a huge mortality burden, but are also reponsbile for significant political and social disruption amongst populations and can negatively affect economic growth in the long-term. Therefore, the importance of strategic planning to limit the negative impact a pandemic would have on NZ cannot be overstated. 


\subsection{Pandemic planning in NZ}

The NZ government and the 20 DHBs have plans in place for how they would manage health and other essential services during an influenza pandemic. The government strategy is set out in the 'New Zealand Influenza Pandemic Plan', which addresses the preparation for, and response to, the threat of an influenza pandemic. The plan is based on modelling for a severe pandemic, where approximately 40 percent of the NZ population would be affected and predicts a fatality rate of two percent, or approximately 38,000 deaths, over an eight-week period (MOH, 2017).

The national plan centers around planning and preparedness, containment, management and recovery and predicts that a moderate to severe influenza pandemic will put significant pressure on EDs. Despite this acknowledgement, the plan states that it is the responsibility of DHBs to plan for their health services to "respond to large volumes of demand in a significant health emergency, while maintaining normal health services to the greatest degree possible" (MOH, 2017, p. 129). One of the only strategies specific to assisting ED to manage this increased demand for services is the establishment of community-bassed assessment centres (CBACs), where lower-acuity patients presenting with influenza-like illness (ILI), can be directed to instead of waiting for assessment and care at ED.

The national pandemic plan relies on DHBs to have robust plans in place for their regions. However, it is significant that the DHB plans for the study site EDs used in this research have little specific information on how these EDs are to manage the expected increase in patients. The existing plan lists an expectation there will be longer ED waiting times, that non-urgent presentations will be redirected to comunity services, and that patients with ILI needing treatment in ED are given masks and grouped as able. The DHB plans are based on an estimate that a severe pandemic will result in a 15 percent reduction in staff numbers due to sickness. With the national plan assuming a case rate of 40 percent in the same scenario, this is an important difference in regional and national planning ( $\left.2018^{1} ; \mathrm{MOH}, 2017\right)$.

\footnotetext{
${ }^{1}$ Specific DHB name removed to maintain site confidentiality
} 


\subsection{Thesis overview}

This first chapter contained a brief overview of the structure of emergency care in NZ, as well as the influenza pandemic plans currently in place at regional and national level. The second chapter presents a review of the literature regarding the impact influenza and other disease pandemics have had on EDs and ED staff, as well as what is known about the concerns nursing staff hold when caring for infectious patients. The methodological principles underpinning this research, study design and methods, research rigour and the ethics approval process are detailed in the third chapter. The findings from this research are presented in the fourth chapter, organised into the key themes and subthemes that emerged from analysis of the raw data. A discussion of the significance of the research findings is outlined in the final chapter, which also provides an overview of the strengths and limitations of the research. The thesis finishes with evidence-based recommendations for EDs, DHBs and the government to ensure the NZ health system is prepared to successfully manage an influenza pandemic. 


\section{CHAPTER TWO: LITERATURE REVIEW}

\subsection{Introduction}

A literature review is an important early step within the research process, allowing the identification of what is known about a subject through a systematic and critical review of published literature. The process helps to inform the direction of future research by identifying gaps in the existing body of knowledge, and allows researchers to review conceptual frameworks and study designs to help guide their methodological decision-making (Elliott, 2007).

\subsection{Literature search}

The databases searched for this review were PubMed, Scopus, CINAHL, Ovid Emcare and Google Scholar. The search terms that yielded the most relevant results were 'influenza', 'pandemic', 'infectious AND disease', 'emergency AND department OR room', 'nurs*' and 'triage'. 'New Zealand' was used as a search term, however no relevant material was uncovered that explored the NZ context. 'NOT vaccin*' was used to exclude results that were primarily about influenza vaccine uptake and efficacy. The search was initially limited to articles published between 2008 and 2018, however the data parameters were extended to include some older studies considered to be of significance within the field. Articles not in English and those without full text access were excluded. Of the 158 articles abstracts that were reviewed to determine relevance to the research question, 46 articles were identified as eligible and read in full. Figure 1 further summarises the eligibility assessment process.

Nineteen articles are included in this review and were conducted in seven countries (Australia, Canada, Chile, Hong Kong, Ireland, Taiwan, United States). The studies involved one or more of the following hospital staff: nurses, doctors, administration clerks, technicians, cleaners, security and paramedics. The articles were a mix of smaller qualitative designs, such as interviews and focus groups, and larger 
quantitative designs, including surveys and case-control series. Five key themes emerged from the literature search: occupational exposure to infectious disease, staff perceptions and concerns, presenteeism, infection control and triage practices and principles.

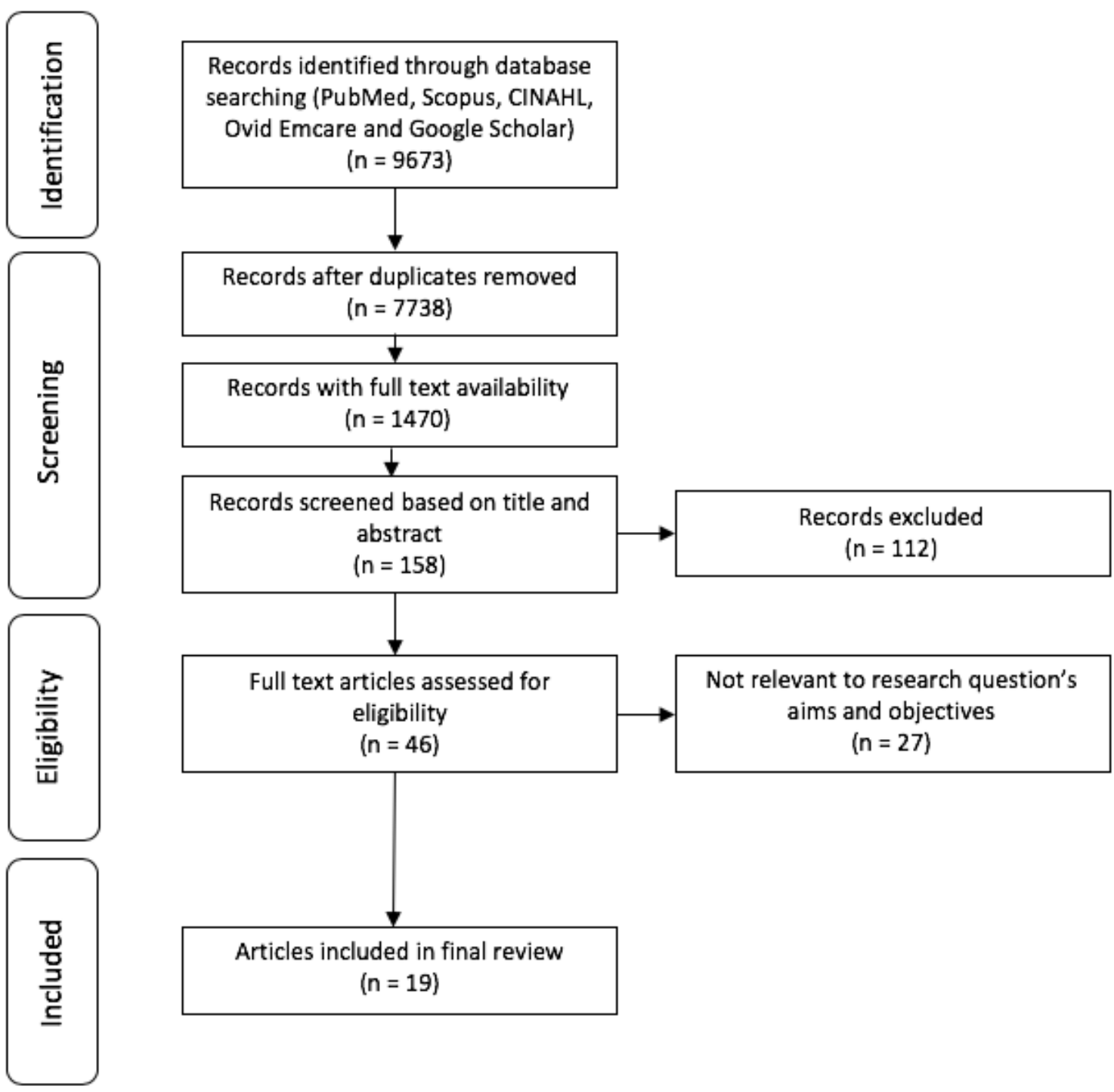

Figure 1: Screening process of retrieved articles, including the stages of excluded articles and the assessment of eligibility

\subsection{Occupational exposure to infectious disease in pandemic scenarios}

EDs are busy environments that are often overcrowded, with patients required to wait in close proximity to others in waiting rooms or treatment area. Patients with undiagnosed infectious diseases may be in close proximity to patients and staff without adequate infection control measures in place, making the ED a high-risk area 
for transmission of infectious diseases (Fusco \& Puro, 2012; Rothman et al., 2006). Three studies have examined how the respiratory viruses responsible for recent pandemics resulted in high numbers of infectious patients presenting to EDs for care, increasing the risk of exposure of ED staff (Sandoval et al., 2016). These include two studies investigating SARS and one study investigating H1N1 influenza.

Occupational exposure to infectious disease was a significant problem during the 2002-03 SARS pandemic, causing major disruption to health services. A Canadian hospital forced to close temporarily due to staff sickness was investigated using a casestudy method to identify how so many healthcare workers were infected with the virus. Varia et al. (2003) found that ED staff were exposed to SARS when an undiagnosed patient spent 18 hours in the ED waiting for an inpatient bed due to hospital access block. This patient infected 11 ED nursing and administration staff, three ED patients and seven people visiting other ED patients. In total, 128 staff, patients and visitors within the hospital were infected directly from exposure to this patient. An additional 16 family and friends of infected ED staff and patients were also infected with the disease (Varia et al., 2003). The researchers note that the proximity of patients and frequent patient movement within the ED, movement of nursing staff between patients and the unrestricted movement of visitors around the ED are all likely to have contributed to the spread of SARS within the ED environment (Varia et al., 2003).

Similarly, a quantitative multiple approach study investigated how 12 staff, including 6 nurses, and 19 patients were exposed to SARS in an urban Taiwanese ED. Contact tracing of patients, staff questionnaires to identify failures in infection control and swabs to identify the virus in the ED environment were undertaken (Chen et al., 2004). The index patient was identified, who presented atypically with symptoms consistent with heart failure. Only six of the staff were found to have cared directly for the index patient or subsequent patients with SARS, and no common source of infection was found that would account for all 12 staff cases of SARS. Subsequently, the researchers hypothesised that some ED staff may have been infected via their environment, so swabs were then taken from around the ED. Of the 119 environmental samples taken, 
nine were positive for the SARS virus (Chen et al., 2004). A limitation of this research is that a copy of the questionnaire used in the survey was not included, making it difficult to determine adequacy (Quick \& Hall, 2015). Furthermore, there was a lack of discussion on potential hindsight bias by using retrospective reporting on infection control measures. The researchers did note that overworked staff may not take adequate precautions and memories can become distorted over time (Chen et al., 2004).

The H1N1 influenza pandemic resulted in a rapid increase in demand for ED services worldwide, placing ED staff at risk for exposure to this virus. Staff in the ED of a Chilean hospital were found to have been more than three times more likely to contract influenza than staff working in the operating theatre (Sandoval et al., 2016). This quantitative prospective comparative study obtained blood sampling from staff working in both areas and screened for virus antibodies. A statistically significant increase in risk for ED staff was found, and a higher rate of antibodies in ED staff samples was noted, suggesting that staff had been exposed to the virus multiple times. A similar study carried out in Australia involving staff working during the same pandemic found that whilst both ED and Intensive Care Unit (ICU) staff showed an increased risk of contracting influenza, ICU staff had higher rates of positive tests (Marshall et al., 2011). However, the Chilean and Australian studies are not directly comparable as they involved different specialty areas, and testing was carried out at different points during the pandemic. The Chilean study samples were obtained the height of the pandemic season, whilst the Australian samples were taken later when the researchers reported the pandemic to be waning (Marshall et al., 2011). This difference in the testing period may contribute to the contrasting results between these two similar studies, although other independent variables (such as use of personal protective equipment (PPE), hand hygiene procedures and staff uptake of influenza vaccines) may also have some impact on the results and thus, direct comparison between these studies cannot be made (Sandoval et al., 2016). In summary, research examining previous SARS and influenza pandemics demonstrate the significance of disease transmission via the ED environment. 


\subsection{Staff perceptions and concerns}

As pandemics are relatively rare events, studies of staff responses to pandemics are divided between those who have worked during a pandemic, and those who have not. The latter have instead been asked scenario-based questions to examine intended behaviours. The key themes that emerged from the research are high workloads, lack of hospital pandemic preparation and the risk of contracting disease and transmitting disease to family members (Corley, Hammond \& Fraser, 2010; Filice et al., 2013; Lam \& Hung, 2013; McMullan, Brown \& O'Sullivan, 2016; O’Boyle, Robertson \& SecorTurner, 2006).

\subsubsection{High workloads}

With EDs already often overworked and under-resourced, the expected increase in people presenting to EDs for care during a pandemic is a major concern of ED nurses across the world (McMullan et al., 2016; Morley, Stankovich, Peterson \& Kinsman, 2018). A qualitative exploratory study investigating the perceptions of 10 emergency nurses in Hong Kong who worked throughout the 2009 H1N1 influenza pandemic found that the significantly increased workload during the pandemic was stressful. However, despite this stress, these nurses continued to attend work and provide care to patients as they feared that nobody else would (Lam \& Hung, 2013). A limitation of this study is the small sample size, meaning researchers did not reach data saturation, and the purposive sampling method used, which is prone to researcher bias.

Difficulty meeting the demands of increased patient volumes and workload was also expressed in a study of Irish nurses when asked about the possibility of the next influenza pandemic. McMullan et al. (2016) designed a quantitative case study using a structured questionnaire distributed to all 127 nurses in an acute care hospital in Ireland to determine staff concerns, with 91 nurses responding. Nurses were concerned about the increase in their workload and the potential lack of extra staff to meet the increased demands a pandemic outbreak would create on the hospital system. McMullan et al. argued that measures to address workload and staffing within pandemic plans was imperative to reducing the negative impact a pandemic would 
have on the psychosocial health of staff (2016). The researchers chose a voluntary questionnaire as their sampling method to meet their objective of identifying the views of all 127 nurses within a single hospital. The high response rate helped to overcome responder bias as a limitation of a voluntary sampling method (Quick \& Hall, 2015). Whilst this study provides valuable insight into the perspectives of nurses in acute care settings, not all participants work within the ED environment and hence, the transferability of this study to the ED context is limited.

\subsubsection{Lack of hospital preparedness}

Many concerns have been raised about how prepared hospitals were when faced with managing a pandemic by staff who worked during the 2009 H1N1 influenza pandemic. A qualitative approach was used to interview 20 paediatric ED physicians across the United States using semi-structured phone interviews. Many hospitals were found to use their mass casulty plans for their pandemic response, however study participants felt these were inadequate as they were not designed for a sustained high presentation rate over a prolonged period of weeks to months, and did not include guidance on reducing disease transmission. This resulted in many EDs being forced to develop other measures on an ad hoc basis (Filice et al., 2013). Other concerns raised included slow service-wide responses to the pandemic, with hospitals not anticipating the increase in ED presentations and delays in the implementation of processes to manage the surges, resulting in increased pressure on the ED. Other concerns raised included public health advice, such as staff needing to wear full PPE when caring for all patients with fever, were not practical in a busy ED envronment and caused frustration (Filice et al., 2013). Of particular note, some hospitals had pandemic plans which included requirements to triage less acute patients away from the ED to primary care facilities to manage surges, however some participants reported that these plans failed due to pushback from primary care providers. Many felt this was due to a lack of awareness or buy-in to the district plans by primary care providers (Filice et al., 2013). 
As this study used medical staff as participants, it remains to be seen if similar concerns would emerge amongst nursing staff, however the study design was documentated thoroughly and included a copy of the interview schedule, enhancing the ability of another researcher to replicate the study amongst ED nurses to determine the existence of similar concerns. Other strengths of this study include the purposeful sampling method, which ensured that a diverse range of hospitals across a wide geographic area and with varying degrees of patient numbers and acuity levels were represented within the sample. This study also achieved credibility by distributing a summary of the results to participants to establish that the results were an accurate reflection of their reality. The researchers note that transferability is limited to paediatric teaching hospitals, and hence the findings are not generalisable to adult and mixed age EDs (Filice et al., 2013).

\subsubsection{Transmission of disease to self and family}

The fear of contracting a contagious disease, and the fear of transmitting disease amongst family members was of significant concern for hospital staff across multiple studies using different methodologies (Lam \& Hung, 2013; McMullan et al., 2016; Corley, Hammond \& Fraser, 2010; O'Boyle, Robertson \& Secor-Turner, 2006). Both the Hong Kong emergency nurses, and the Irish hospital nurses in previously discussed studies expressed concern about the risk to their health from occupational exposure to influenza, as well as fearing spreading the disease to family members (Lam \& Hung, 2013; McMullan et al., 2016).

The fear of becoming sick or spreading disease to family members was also evident in a Heideggerian phenomenological study of the lived experience of 34 nursing and medical staff in an Australian ICU during the 2009 H1N1 influenza pandemic. Staff were reluctant to care for infected patients, and some staff feared transmitting the virus to their children, which manifested in anxiety about being touched by family members after a shift (Corley et al., 2010). One limitation of this study is the question of how generalisable the findings are to other areas of nursing, however detailed 
information on the ICU used in this study helps to determine transferability (Corley et al., 2010).

O'Boyle et al. (2006) investigated how nurses felt about their risk of exposure to lethal infectious disease from a bioterrorism perspective rather than a pandemic. This qualitative study used focus groups to determine their perspectives, and the interventions that nurses felt would aid them in their ability to cope with their role during a bioterrorism event. Researchers recruited 33 nurses from EDs, critical care units and medical-surgical wards across three designated bioterrorism recieving hospitals. Nurses held fears about disease exposure, transmission of disease to their families, and managing panicked members of the public demanding access to medications and PPE. They felt public health staff should be on site to offer information and advice on reducing disease transmission, and hospital management should take a greater role in communicating with the public and controlling hospital access. Nurses also wanted a system developed to help ensure adequate staffing during a bioterrorism emergency, with one nurse noting "we can hardly manage now. We could not cope with a great surge of patients" (O'Boyle et al., 2006, p. 350). Finally, nurses felt that hospitals should be committed to supporting staff by ensuring the families of nursing staff are given access to prophylactic medication, noting that there should be enough "to cover your family so you know that you're not sacrificing more than yourself when you come in and work during that time" (p. 351). As this study was about a bioterrorism situation rather than a naturally-occuring pandemic, it must be acknowledged that nurse responses may differ between the two events. Furtheremore, the transferability of this study to the NZ or other contexts is made difficult by the lack of information about how participants were recruited, how the study sites were selected, or information on the characteristics of these hospitals.

\subsection{Presenteeism}

With increased exposure to infectious disease as an occupational hazard of working in an ED, many hospitals would expect to see an increase in absenteeism of staff due to illness. However, the opposite has been found to be true in research undertaken in 
Australia, where staff were found unwilling to take time off work, despite knowing the risk of transmitting disease by doing so (Considine et al., 2011; Seale, Leask, Po \& Maclntyre, 2009, Arbon et al., 2013). This phenomenon has been called 'presenteeism' and has been shown to negatively impact on staff productivity (Rantanen \& Tuominen, 2011).

A large cross-sectional quantitative survey of Australian ED medical and nursing staff who worked during the H1N1 influenza pandemic was undertaken to investigate staff concerns of working during the pandemic, and if the increased risk of exposure to disease correlated to an increase in absenteeism (Considine et al., 2011). Of the 618 respondents, $37 \%$ reported becoming unwell with influenza-like illness (ILI) symptoms during the pandemic. However, the majority were not tested and therefore it cannot be inferred that all of the unwell staff had H1N1 influenza (Considine et al., 2011). Of the staff who reported ILI symptoms, almost half did not take any sick leave from work. This is a significant finding as the infectious period for $\mathrm{H} 1 \mathrm{~N} 1$ has been estimated as being between one to four days, with virus shedding sometimes occurring for up to seven days, and therefore there is a possibility that staff attended work whilst still shedding the active virus (Boehm, Cabral, Harkinson \& Sakers, 2010). Only a small percentage of respondents expressed concern about spreading the virus to their family members, which the researchers considered could be due to the staff feeling a need to respond to a health-care disaster and prioritising their responsibility to provide care over their own personal fears and concerns (Considine et al., 2011). Whilst the large sample size is a strength of this study, the researchers acknowledge is the use of a voluntary web-based survey method is a limitation, due to the potential for responder bias.

Presenteeism was also explored in another Australian study carried out prior to the 2009 H1N1 pandemic (Seale et al., 2009). A cross-sectional study using a questionnaire was carried out across two tertiary-level hospitals in Sydney. Forty departments were randomly selected to create a convenience sample of 1200, with nursing staff representing almost half of respondents. Almost one-quarter of respondents would continue to work after developing ILI symptoms if there was a shortage of staff, 
despite being aware of the risk of transmitting the virus to others by doing so. This reluctance to take sick leave correlates with the Considine et al. (2011) study, though it must be noted that the 2011 study investigated absenteeism in those who worked through a true pandemic, whilst the Seale et al. study was of intended behaviour. The questionnaire used was pilot tested before use to help ensure the validity of the tool. The large sample size and diverse representation of multiple healthcare occupations are also significant strengths, however the transferability to the ED context is again limited as the sample includes staff across many acute care specialties.

The use of these quantitative approaches has produced valuable information around ED and hospital staff behaviours and their use of sick leave, however these studies do not explore the factors that contribute to staff decision-making. Arbon et al. (2013) recognised the need to gain a greater understanding of these factors and carried out a mixed-methods study of Australian ED nurses to determine their willingness to attend work in a disaster. The research found the nurses felt torn between their moral obligation to work during an infectious disease outbreak and a personal concern for their own and their families' safety. They were more likely to attend work if their workplace had a detailed plan in place that specified how their role might change in the event of a pandemic and would also like the ability to be able to communicate with their families from work. ED nurses further identified concerns about a potential slow response from the rest of their hospital and how this would impact on their EDs that are already over-stretched and under-resourced (Arbon et al., 2013). This rigorous study used an initial survey of 451 nurses to determine the factors influencing nurses' willingness to work. This information was then used to guide the tools used by researchers in the focus groups and interviews of 41 nurses. Whilst the rich descriptions produced within this research is valuable, it is about disasters broadly, rather than pandemics specifically.

\subsection{Infection control}

Triage has been identified as the highest risk area for infectious disease transmission within the ED setting (Martel et al., 2013). Compliance with infection control measures 
by triage nurses can help to prevent the spread of infectious diseases within the hospital. However, a cross-sectional study investigating respiratory hygiene practices within the triage area of two Canadian EDs showed that compliance with infection control measures was low (Martel et al., 2013). The triage of 118 patients presenting with fever and cough was observed across all shifts during one week during the seasonal influenza period. Nine variables regarding identification and isolation of patients presenting with respiratory infection symptoms were evaluated by the research team. Whilst most nurses asked about high-risk symptoms as a means of identifying infectious patients, only $12 \%$ of patients were isolated from others. Likewise, only $18 \%$ of patients were asked to wear a mask, and $9 \%$ were shown proper mask technique. Additionally, nurses were observed to display poor adherence to hand hygiene practices, with only half washing their hands after patient contact.

A questionnaire was distributed to the staff at these EDs to ascertain their perceived infection control knowledge and practices. The tool was developed from existing, validated literature and a Likert scale was used to obtain the data. Ninety-two percent of staff reported the use of a mask was an effective infection control measure to prevent disease transmission and $94 \%$ reported they would recommend to a patient with fever and cough to wear a mask. The high perceived compliance rate does not correlate with the small number of patients asked to wear a mask in the observational study. The researchers note that there may be a social desirability bias and a tendency to overestimate skills within the questionnaire responses. Another limitation is that ethical approval was dependent on the questionnaire being anonymous, providing no way to correlate the questionnaire results of the triage staff observed during the study. This study identified an important variance between perceived and observed infection control practices and represents a need for further staff education and training.

\subsection{Triage practices and principles}

Triage practices are known to change dramatically in the face of an infectious disease emergency. The process of triaging away is included in the pandemic plans of both NZ and other countries, and is centered around the diversion of stable patients with ILI 
away from the ED as a means of isolating the potentially infectious, as well as relieving some of the ED workload during a pandemic (Australian Government Department of Health, 2019; $\mathrm{MOH}, 2017)$. To investigate staff awareness of triage processes, Rottman et al. (2010) carried out a qualitative study of staff from multiple hospitals across Los Angeles using structured interviews. Participants were recruited using a non-random snowball sampling technique, and consequently, the results of this study may not be applicable to other areas outside of the study centres due to anchoring bias. Anchoring bias occurs when an individual uses the initial information gathered to interpret any subsequent data (Croskerry, 2002; Quick \& Hall, 2015).

With an influenza pandemic expected to cause large numbers of patients to overwhelm EDs, Rottman et al. asked participants about how this would affect normal triage practice and accepted standards of care. The participants initially expressed confidence that triage would continue to operate as normal and that the standard of care patients received within their department would continue to be high. Participants were then informed of the possibility that services would be so overwhelmed that their department could not operate as normal and some patients may have to be triaged away in a pandemic. When faced with this scenario, participants expressed concern about sending patients away and felt underprepared and unqualified to do so. Participants described a conflict between their duty to provide care to all patients in need and their duty to adhere to hospital procedures designed to manage patients with the greatest need when resources are overwhelmed. A limitation is that there could be a question of leading or coaching of the participants in this study, as the authors note that they were sometimes required to reframe their questions and ethical scenarios multiple times before they reached the answers they used in their discussion. However, the themes that emerged from this study are important and require further exploration, most critically the ethical dilemma for ED nurses when they and their department can no longer provide the expected standard of care due to the volume of patients overwhelming the service.

Triaging away was used at several Australian hospitals during the H1N1 influenza pandemic, where influenza assessment clinics (IAC) were established to receive 
diverted patients. Ranse, Lenson, Luther and Xiao (2010) used a retrospective descriptive design to evaluate the caseloads of both the ED and the IAC. The majority of patients were correctly triaged and diverted to the IAC, with only $2 \%$ of IAC patients requiring hospital admission. However, only $23 \%$ of ED patients with ILI were admitted to the hospital, meaning there were still relatively well patients being treated and discharged from the ED that could potentially have been diverted to decrease the overall ED workload. There was no information about how patients were separated into the ED and IAC streams by the triage nurse and this lack of information on the triage process affects the validity of the results of this study as it cannot be confirmed that a routine process was followed for each patient. The authors note that the IAC model could be adapted for use in any scenario where there is likely to be a significant surge in ED presentations, such as mass casualty situations.

A similar process was carried out by one large, urban paediatric ED in the United States to manage increased presentations during the H1N1 pandemic. Triage nurses would carry out a standardised screening process on all patients presenting with any ILI symptoms and those considered well were sent to a rapid screening unit (Fagbuyi et al., 2011). The advantages of this study over the Ranse et al. (2010) study in Australia is that ILI symptoms were clearly defined and the screening tool used for sending patients to the rapid screening unit was included in the study. In contrast to the Australian study, only one of 1767 patients who were sent to the rapid screening unit required admission to hospital (Fagbuyi et al., 2011). The large sample size of this study is a strength when interpreting the results, however the authors note that their study was only carried out across one institution and may not be replicable elsewhere, and that their influenza diagnoses were based on clinical findings rather than serology testing, so there is the possibility that known complications of influenza, such as pneumonia, were incorrectly coded as non-ILI patients and this may affect the results (Fagbuyi et al., 2011). However, serology testing in not necessarily feasible for all patients presenting with ILI and therefore this study remains valid. 


\subsection{Strengths, weaknesses and gaps}

In summary, this literature review uncovered five recurring themes with regards to nurses working during an influenza pandemic. These themes are occupational exposure to infectious disease in a pandemic scenario, staff perceptions and concerns, presenteeism, infection control and triage practices and principles. Many of the studies in this review focussed on nurses working in other specialties, despite ED nurses being at higher risk for exposure to infectious disease through their work. The majority of the research have been quantitative surveys of staff, and although these have produced important information into the intended and actual behaviours of nurses working during a pandemic, they do not provide insight into nurses decisionmaking. Understanding why nurses choose to attend work knowing they are at increased risk, continue to work through sickness or knowingly under-utilise infection control precautions is crucial to addressing the contributing factors behind these risky behaviours. Whilst research has been undertaken into each of these themes previously, no studies have investigated all themes cohesively within a single study of ED nurses in order to provide detailed information on their unique perspectives. Additionally, no studies have been carried out that explore the NZ ED context. 


\section{CHAPTER THREE: METHODOLOGY AND METHODS}

\subsection{Introduction}

In this chapter the methodology and study design are presented. The perspectives of ED nurses around pandemic preparedness and planning were examined using a qualitative descriptive research design. The methods used in this study are outlined, detailing the processes of data collection, management and analysis. Ethical considerations, the process of reflexivity and strategies employed to ensure the trustworthiness of the study findings complete the chapter.

\subsection{Study aims and objectives}

This research was designed to address the question of what are the strategies and processes ED nurses consider important to safely manage during an influenza pandemic. The research aimed to identify nurses' perspectives around the provision of nursing care during a pandemic, and their perspectives around the safety of themselves, their families and of ED patients. By identifying the perspectives of these nurses, this research aimed to further identify strategies to improve nursing care during a pandemic and to inform future pandemic planning for NZ EDs.

\subsection{Methodology}

Research can be divided into two general classifications: quantitative and qualitative research. Quantitative research is the objective measurement of variables that can be quantified as numerical data. Qualitative research has been defined in its most basic form as the use of words as data (Whitehead, 2007). Within these classifications exist paradigms, or patterns of beliefs and practices, that guide how knowledge should be generated and what knowledge is considered meaningful (Weaver \& Olson, 2006).

Both quantitative and qualitative designs were included and analysed in the literature review to determine which methodology and method produced the most relevant information pertaining to the subject of ED nurse perspectives around working during 
an influenza pandemic. With identification of these perspectives within the NZ context set as the overall aim of the research, but no specific independent variable within this aim to measure, a non-experimental design was chosen (Elliott \& Thompson, 2007). Whilst quantitative studies using large-scale surveys did elicit useful data around the subject, they failed to provide information on the contributing factors governing nurses' perspectives and therefore made it difficult to infer what strategies service providers could employ to meet the needs of EDs and ED nursing staff. The lack of any similar studies carried out within the NZ context meant there is no NZ-based research to build upon or use as a guide, so a qualitative approach was deemed the most appropriate methodology for this study.

The qualitative approach allows the researcher to take an inductive approach and build up from information provided first-hand by nurses, exploring their perspectives in-depth and identifying strategies that may inform future pandemic plans in NZ (Whitehead, 2007). Qualitative research explores the views, perspectives and experiences expressed by participants to understand their interpretation of the phenomena being studied. This approach allows the researcher to "make sense of how the world is seen, understood and experienced from the person's perspective" (Clarke \& Braun, 2013, p. 24). Additionally, this study used an interpretivist paradigm, which focuses on the meaning behind the actions of individuals and examines the importance of context within research (Whitehead, 2007).

\subsubsection{Descriptive, exploratory and interpretive designs}

Within the realm of qualitative enquiry exist a range of methodological frameworks that can inform a research design. Descriptive, exploratory and interpretive qualitative designs began to emerge more recently as researchers began to recognise that meaningful knowledge can be derived from personal experience without being viewed through a prescribed theoretical framework (Thorne, 2014). The goal of qualitative description is to produce "a comprehensive summary of events in the everyday terms of those events" and provide a 'rich' description of the phenomena of interest (Sandelowski, 2000, p. 334). The method is well suited to exploring the first-hand 
accounts of health professionals' experiences of a phenomena, and for identifying important considerations of a phenomena which has limited previous exploration (Elliot \& Timulak, 2005; Neergaard, Olesen, Andersen \& Sondergaard, 2009). Whilst descriptions are influenced by the perceptions and opinions of the researcher and the choice of what data is reported, the method is still considered low-inference. The aim is to remain as close to the data as possible, which can make it easier to achieve consensus between researchers (Sandelowski, 2000).

Qualitative exploratory design is also useful for investigating phenomena where there are few or no existing studies. The overall aim is discovery, where the researcher gains insights that may be used to determine the how and why of a phenomena (Brink, 1998). This differs to qualitative description, which tends to focus on the "who, what and where of events or experiences" (Sandelowski, 2000, p. 338). Qualitative exploratory design often employs an unstructured interview technique which allows the researcher the flexibility to ask further questions as they emerge from the interview responses (Brinkman, 2014). Finally, a qualitative interpretive design "acknowledges the constructed and contextual nature of much of the health-illness experience" (Thorne, Kirkham \& MacDonald-Emes, 1997, p. 172). Thorne et al. postulate that qualitative interpretive design is appropriate where there is existing knowledge around a subject that can be expanded upon to generate new meaning by examining context and language.

The choice of qualitative descriptive design is appropriate for an investigation into the chosen phenomena in NZ. The lack of any previous study makes qualitative interpretive design unsuitable, and whilst the use of a qualitative exploratory design was considered, the unstructured nature of data collection was considered challenging for a new researcher who would benefit from the guidance of the semistructured interview format and the low-inference nature of the study design. 


\subsection{Study design}

Qualitative descriptive design is compatible with a range of methods to obtain data, such as interviews, focus groups and participant observation (Thorne, 2014). Interviews are the most common method of qualitative data collection and are well suited to exploring a participant's understandings or perceptions of a phenomena in which they take a particular personal interest, allowing for the collection of rich descriptions that focus groups or surveys do not often achieve (Clarke \& Braun, 2013). The aim is to capture the thoughts, feelings and opinions of a participant in their own words, and use these to build a picture of a participant's reality. Successful interviewing requires a partnership between the researcher and the participant built on mutual trust and active listening that allows for mutual disclosure (Liamputtong, 2013). With a broader research question where little is known on a topic, taking a flexible approach to interviews by employing the semi-structured method allows participants to determine what knowledge they consider meaningful. The researcher develops a list of questions around the research topic but allows opportunities for the participant to discuss issues that they feel are important and that the researcher may not have anticipated (Clarke \& Braun, 2013; Liamputtong, 2013). The semi-structured interview method was selected for use in this study.

\subsection{Study setting}

The study setting were two large EDs within the North Island of NZ. Site one is a tertiary-level facility with an annual presentation rate of approximately 65,000 patients. Site two is a secondary-level facility that cares for approximately 48,000 patients per year. Using two sites allowed for recruitment of participants from EDs reflective of varying levels of hospital services available throughout NZ (MOH, 2015).

\subsection{Participant selection, recruitment and sampling strategy}

The inclusion criteria for this study was registered nurses working in ED at either study site who were trained to work in triage. ED nurses who did not work in triage were excluded from the study. Participants were not excluded based on gender, ethnicity or age. 
Following ethics approval, locality approval was sought from the respective sites' DHBs (Appendix 1). Recruitment occurred via two processes and both involved the permission of each ED Nurse Manager.The first process of recruitment was via an email which was sent out to the nursing staff of both study sites (Appendix 2). The email contained an overview of the research question, information on participant inclusion criteria, data collection methods and the proposed timeframe of the research. The email concluded with an invitation for interested nursing staff to participate and detailed how to contact the researcher. In addition, posters with information about the study were placed in the staff room of each ED to allow for further exposure of the study to nursing staff (Appendix 3). Respondents who expressed interest in participating were then emailed the information sheet for them to review and discuss with friends and family. Throughout the recruitment process, potential participants were made aware that this was a voluntary study.

Sample size can vary between qualitative studies and no fixed rule applies. Clarke and Braun (2013) note that the sample must be of sufficient size to demonstrate patterns across the data, yet small enough that individual voices are not lost. Normally a small study using interviews and thematic analysis to collect and analyse data involves between 6 to 10 participants, whilst a medium study usually involves between 10 and 20 participants (Clarke \& Braun, 2013). A convenience sample of 16 nurses across both sites, from a total pool of approximately 80 triage-trained nurses, was chosen in order to answer the broader research question with meaningful depth. Convenience sampling allows researchers to access participants who are located close at hand and willing to participate (Liamputtong, 2013). Convenience sampling was chosen as this meant the researcher could meet participants face-to-face without increasing cost and travel time for the researcher (Clarke \& Braun, 2013).

\subsection{Data collection}

Data collection was undertaken through a semi-structured, face-to-face interview with each participant. Firstly, demographic information on age, gender, ethnicity, 
place of nursing registration, years since nursing registration, and years of ED and triage experience were collected to establish the diversity of the sample and help determine the transferability of the findings (Clarke \& Braun, 2013; Nowell, Norris, White \& Moules, 2017) (Appendix 4). Information was further collected on family dependents, such as children and those with co-morbidities, as this could provide insight into the beliefs about nursing influenza patients and actions described by participants during an influenza pandemic. Questions specific to the research topic were then asked, clustered into logical sections reflecting the themes identified in the literature review (Appendix 5).

Questions addressed concepts such as nurses' perspectives on how their EDs manage seasonal influenza and how they would manage in a pandemic; nurses' decisionmaking around taking sick leave; the implementation of infection-control principles; the management of influenza patients at triage; and the risks associated with caring for influenza patients. The questions were written in consultation with the research supervisor to ensure they were open questions, and the interview schedule was submitted to the ethics committee before commencement of data collection to ensure they were not considered leading or coercive. The use of open questions allowed participants to determine what knowledge on the topic is meaningful (Liamputtong, 2013). The interviews were carried out in a location where the participant felt comfortable and where the researcher felt safe, such as the participant's workplace or home. The researcher ensured that the supervisor was aware of the interview time and place beforehand to help ensure researcher safety (Clarke \& Braun, 2013). Interviews were audio recorded using a dictaphone and transcribed verbatim. This was clearly outlined in the consent process and audio recordings and transcriptions were securely stored.

\subsection{Data management}

To maintain the ethical principle of respect for privacy, all electronic information and data collected during the study has been stored in a password-protected file, and all hard-copies of information, including consent forms, have been stored in a locked 
filing system with access limited to the researcher. All information will be destroyed after five years, as outlined within the consent process. Whilst the researcher will store and manage the data as outlined for the purposes of the study, it is important to consider that data legally still belongs to the individuals who provide it (Lincoln, 2009). In respect of this, and to help ensure study credibility, the researcher elected to offer participants the option of receiving a transcript of their interview and allowed them to comment on the data they provided. Participants had this option explained to them during the informed consent process to determine if they would like to receive their transcript, however no participants chose to review their interview transcript.

\subsection{Data analysis}

The aim of qualitative descriptive design is to present a "rich" description of the phenomena of interest whilst staying as close to the data as possible, and this influenced the decision to utilise both content and thematic analysis methods for data analysis in this study (Sandelowski, 2000, p. 334). Qualitative description is based on the factist perspective, which assumes "data to be more or less accurate and truthful indexes of reality" (Sandelowski, 2010, p. 80). Both content and thematic analysis are also grounded within this perspective, and are hence considered suitable methods to analyse descriptive data (Sandelowski, 2010).

Content analysis is a method used to analyse verbal, written or visual data (Elo \& Kyngäs, 2008). It requires the researcher to immerse themselves by reading all of the data to achieve a "sense of the whole", before codes are extracted from the words used by participants that capture important concepts (Hsieh \& Shannon, 2005, p. 1279). It allows the researcher to make cogent and replicable inferences from the data. and can take either an inductive or deductive approach. An inductive approach is suitable when little is known about a phenomenon and was used in this research. Codes and categories were built up from the data, starting with specific data points that are then linked to create a general concept (Appendix 6). Content analysis can be used "in parallel" with other methods, often as a preliminary method to allow the 
researcher to get an idea of the entire data set, before the researcher moves into more specific analysis (Prior, 2014, p. 361). The use of content analysis allows the researcher to ensure they are taking into account the full breadth of the data, rather than immediately focussing on selected extracts and overlooking those which offer a different view on the same subject matter, which allows for more representative reporting of findings (Prior, 2014). The suitability of inductive content analysis for use in studies with little previous research is an important consideration of why the method was selected for this research.

Thematic analysis takes an inductive approach to identify patterns and wider themes across the data that reflect the research question (Clarke \& Braun, 2013). The inductive approach means the themes developed are strongly data-driven and less guided by the researcher's own analytical and theoretical preconceptions (Braun \& Clarke, 2008). Whilst content analysis is useful for describing the characteristics of a data set and identifying common issues to generate initial codes directly from the data, thematic analysis looks for patterns of codes across the data set and how these relate to each other (Vaismoradi, Turunen \& Bondas, 2013). Braun and Clarke's six phases of thematic analysis were used within this research. Familiarisation with the data occurred through transcription of the data verbatim, and reading and re-reading of the data. Initial codes were developed from similarities and differences in the data pertinent to the research question (Appendix 7). Codes were reviewed to determine how they may combine into a broader theme or small sub-theme. These themes were checked against the data set to ensure an accurate reflection of the overall picture. The researcher then identified the crux of the theme and determined how it fits with other themes, sub-themes and with the dataset as a whole. The themes were then finalised (Braun \& Clarke, 2008).

NVivo 12 coding software was used for both content and thematic analysis. Content analysis produced codes identifying the frequency of words and concepts mentioned across the data set. Each of these content codes was then found to have been reproduced within the thematic analysis of the data and expanded upon, by showing their meaning within the context in which it was said (Joffe \& Yardley, 2004). Data 
from each site was initially analysed separately, but were presented together when no new themes emerged between sites.

\subsection{Ethical approval process}

The ethical approval process is crucial to protect research participants and ensure any harm or discomfort is minimised. Health researchers have "a duty to protect the life, health, privacy and dignity of the human participants" (Coup \& Schneider, 2007, p. 82). The respect for human dignity and the protection of the rights and freedoms of research participants are fundamental considerations taken during the ethical approval process (Coup \& Schneider, 2007). Ethics approval was obtained from Victoria University of Wellington's Human Ethics Committee (HEC) prior to undertaking the research (HEC approval 0000027012) (Appendix 8). The principles of ethical research have been incorporated into the study design.

\subsubsection{Informed Consent}

Informed consent was sought and obtained from all participants before data collection took place. The informed consent process maintains respect for autonomy and individual responsibility by providing sufficient information, answering all questions and allowing for time for discussion with friends and family before a decision is made on whether to participate in the study. All interested participants were emailed an information sheet (Appendix 9) to review before the interview time was arranged. Before commencement of the interview, the researcher went through the consent form (Appendix 10) and participants were asked to sign this if they agreed to proceed. The recruitment process stated that participation in the study was voluntary and any participation, non-participation or information revealed in the interview should not result in any adverse impact upon the participant's employment status. This was important to emphasise as the researcher is employed at site one and some participants are colleagues of the researcher. These colleagues were aware that the researcher held an independent role with a focus on patient movement within the hospital and held no line management or disciplinary responsibilities. Additionally, within the information sheet and the consent form, participants were advised of their 
right to withdraw from the study and the date by which this could occur in order to meet the principle of respect for self-determination (Coup \& Schneider, 2007).

\subsubsection{Minimisation of harm}

The ethical principles of justice and beneficence refer to the equal treatment of all participants, the fair distribution of risks and benefits of the study, and the minimisation of harm to participants (Coup \& Schneider, 2007). Risk in this research was the potential for emotional harm during the discussion of topics that may be distressing. To mitigate this, the researcher ensured that participants were provided with information on how to contact counselling services if necessary. The participants were ED nurses who work in the triage area and were not recruited from a specifically identified vulnerable group. However, the researcher acknowledges that power imbalance can create vulnerability within a group and all participants were treated with respect, regardless of their seniority within the nursing group, in order to meet the principle of respect for human vulnerability and personal integrity (Coup \& Schneider, 2007).

\subsection{Treaty of Waitangi and respect for cultural diversity}

When conducting research in NZ, researchers should ensure that their work respects and incorporates the principles of the Treaty of Waitangi: Partnership, Protection, Participation and Tino Rangatiratanga (Nursing Council of New Zealand, 2011). The research did not specifically include or exclude Māori participants, however Māori are an important part of the NZ population and it is crucial that their specific needs were considered. The choice of a qualitative, semi-structured interview method is appropriate for Māori as it allows for Māori to actively participate and for their voice to be heard. The researcher worked in partnership with Māori by recognising their role as partners in research, and by ensuring that tikanga Māori, or Māori practices and protocols, were respected. The researcher endeavoured to protect Māori rights by recognising that the research may benefit Māori by reducing the burden of infectious disease and improve Māori health outcomes (Hudson \& Russell, 2009). This

is significant, as previous influenza pandemics in New Zealand have had a 
disproportionate impact on Māori, worsening pre-exisiting health inequity within the population (Hobbs et al., 2019; $\mathrm{MOH}, 2017)$. Consultation from Māori was sought before participant recruitment and data collection was undertaken. As NZ is a multicultural society, researchers must be aware of the many cultures that exist within the population and show respect for cultural diversity. The researcher acknowledged that participants may be members of various cultural groups that inform their values and sense of self, such as through ethnicity, religion or sexual orientation. The researcher strived to conduct themselves in a non-judgemental way and recognised the importance of self-reflection to ensure data analysis concentrated on the participants' experience and was not influenced by the researcher's cultural viewpoint (Coup \& Schneider, 2007).

\subsection{Protecting the identities of the study participants}

To ensure confidentiality, the researcher must ensure that a participant's identity is not able to be linked to any information they provide for the study (Coup \& Schneider, 2007). To do this, the researcher undertook processes to de-identify all participants. Care was taken to ensure names were not used during the audio recording of the interviews, and any identifying characteristics were removed during the transcription process. As the recruitment area was limited to two EDs within a defined geographical region, the researcher also took care to remove any contextual details that may have allowed for identification of a participant's workplace to further maintain confidentiality (Traianou, 2014). During transcription, names were replaced with 'participant' followed by a number assigned to them by the researcher. Access to identifying information was limited to only the researcher, whilst access to deidentified information was expanded to include the research supervisor.

\subsection{Rigour and trustworthiness of the research process}

Rigour describes the quality of research and the strength of the research design. As qualitative research is concerned with people and narratives, rather than numbers and statistics, this concept can be challenging to measure (Polit \& Beck, 2004). Rigour in qualitative research involves being thorough in data collection, adhering to 
identified philosophical perspectives in data analysis and openness and accuracy in data reporting (Cypress, 2017). Trustworthiness is a term proposed by Lincoln and Guba as the qualitative alternative to quantitative validity and provides criterion by which to determine the overall rigour of a study (1985). Trustworthiness denotes the quality of the research and how well the findings reflect the truths, allowing the reader to have confidence in the results. Trustworthiness is determined by credibility, transferability, dependability and confirmability (Shenton, 2004).

Credibility is the accurate description and interpretation of the participant's experience, or how well the findings reflect reality. This was achieved by dedicating sufficient interview time to build trust with participants to obtain the most in-depth and accurate understanding of the perspectives of ED nurses around pandemic preparedness and management in NZ (Cypress, 2017). The interviews were audiorecorded to ensure accuracy and the transcribed material was thoroughly checked to ensure interviews were documented verbatim. As findings were made, the researcher returned frequently to the verbatim transcriptions to ensure all conclusions made were grounded within the raw data (Koch, 2006). All participants were offered the opportunity to undertake member checking of their transcripts, however this was not taken up by any participants.

Transferability describes the degree to which the findings could apply to other contexts. This was achieved through the researcher providing a detailed description of the study methods and the context from which the data was gathered, meticulous transcription of material verbatim, and consistent application of qualitative content and thematic analysis techniques. Information on the boundaries of the study, including the sites, inclusion and exclusion criteria, the number of participants, data collection method and the time period of the study have been provided (Nowell et al., 2017; Shenton, 2004).

Dependability refers to the consistency shown by the researcher throughout the research process. This is achieved with a logical and well documented research process and consistent application of the methodological concepts underpinning the 
research (Liamputtong, 2013). Content and thematic analysis were used for data analysis and the supervisor guiding this work is familiar with these approaches and verified coding and analysis (Cypress, 2017; Nowell et al., 2017; Shenton, 2004).

Confirmability is a measure of how well the findings reflect the participant's words, rather than the researcher's thoughts and opinions. The use of an audit trail allowed the researcher to document decision-making during data collection and analysis, and their impression of any developing patterns, themes or theories. These decisions were later reflected upon and discussed with the research supervisor to ensure the findings were grounded in the participant's narrative, rather than the researchers. The use of a detailed audit trail also helped to enhance both credibility and dependability (Shenton, 2004).

\subsection{Reflexivity}

The use of a reflexive account was another method used to contribute to the overall rigour of the research study. Reflexivity acknowledges the influence that researchers have on how the data is analysed and interpreted (Jootun, McGhee \& Marland, 2009). The nature of qualitative research means it is impossible for the researcher to remain completely objective as researchers will instinctively bring their own experiences and perspectives into a study, whether consciously or not (Liamputtong, 2013). Reflexivity is the process by which researchers reflect on their role in shaping the data in order to understand their influence on the findings (Jootun, McGhee \& Marland, 2009). An awareness of the degree to which the research has been influenced by the researcher adds to the credibility, and therefore trustworthiness, of the study (Jootun, McGhee \& Marland, 2013).

As both the researcher and the participants in the study were ED nurses working within a defined geographical region, the term 'insider research' can be applied. Insider research is when a researcher "conducts studies with populations, communities, and identity groups of which they are also members" (Asselin, 2003, p. 100). This can be advantageous in that the researcher is already knowledgeable about 
the research setting and any unique language or terms used within that speciality, and it can be easier to build a rapport with participants. However, the researcher can rely on pre-existing assumptions and thereby overlook important data about the phenomena of interest because of their preconceptions. The parallel roles of researcher and ED nurse were acknowledged and reflected upon by the researcher, and data analysis and findings were reviewed by the supervisor to limit insider bias (Asselin, 2003).

\subsection{Chapter summary}

This chapter provides the rationale for the use of a qualitative methodology in order to answer the question of what strategies and processes do ED nurses consider important to safely manage during an influenza pandemic. With little existing research to use as a guide, a qualitative descriptive design was selected as the methodology. This allowed for the rich and accurate description of the perspectives of ED nurses when faced with working on the frontlines of the NZ healthcare system during a pandemic. Ethics approval was granted before the commencement of recruitment and data collection. Data collection was undertaken using semi-structured, face-toface interviews and data analysed using content and thematic analysis. Ethical principles and respect for the Treaty of Waitangi were incorporated into the research design, and the use of an audit trail aided in producing a rigorous and trustworthy study, the findings of which will be discussed in the following chapter. 


\section{CHAPTER FOUR: FINDINGS}

\subsection{Introduction}

The findings chapter introduces the participants and presents the three themes that emerged from the analysis of the interviews with nurses working in two EDs in NZ (Figure 2). The first theme, 'the everyday reality for ED nurses', highlights current safety issues that exist in emergency nursing today and features three sub-themes 'safety of the patient', 'safety of the nurse' and 'safety at organisational level'. These safety issues provide the foundation for the second theme, 'fears for a future pandemic', where nurses discussed their fears about what could happen to themselves, their patients, and the health system during an influenza pandemic. The final theme, 'strategy and planning', presents the strategies nurses feel are necessary to successfully manage an influenza pandemic in NZ EDs. These strategies are explored under the subthemes of 'ED level', 'DHB level' and 'government level'.

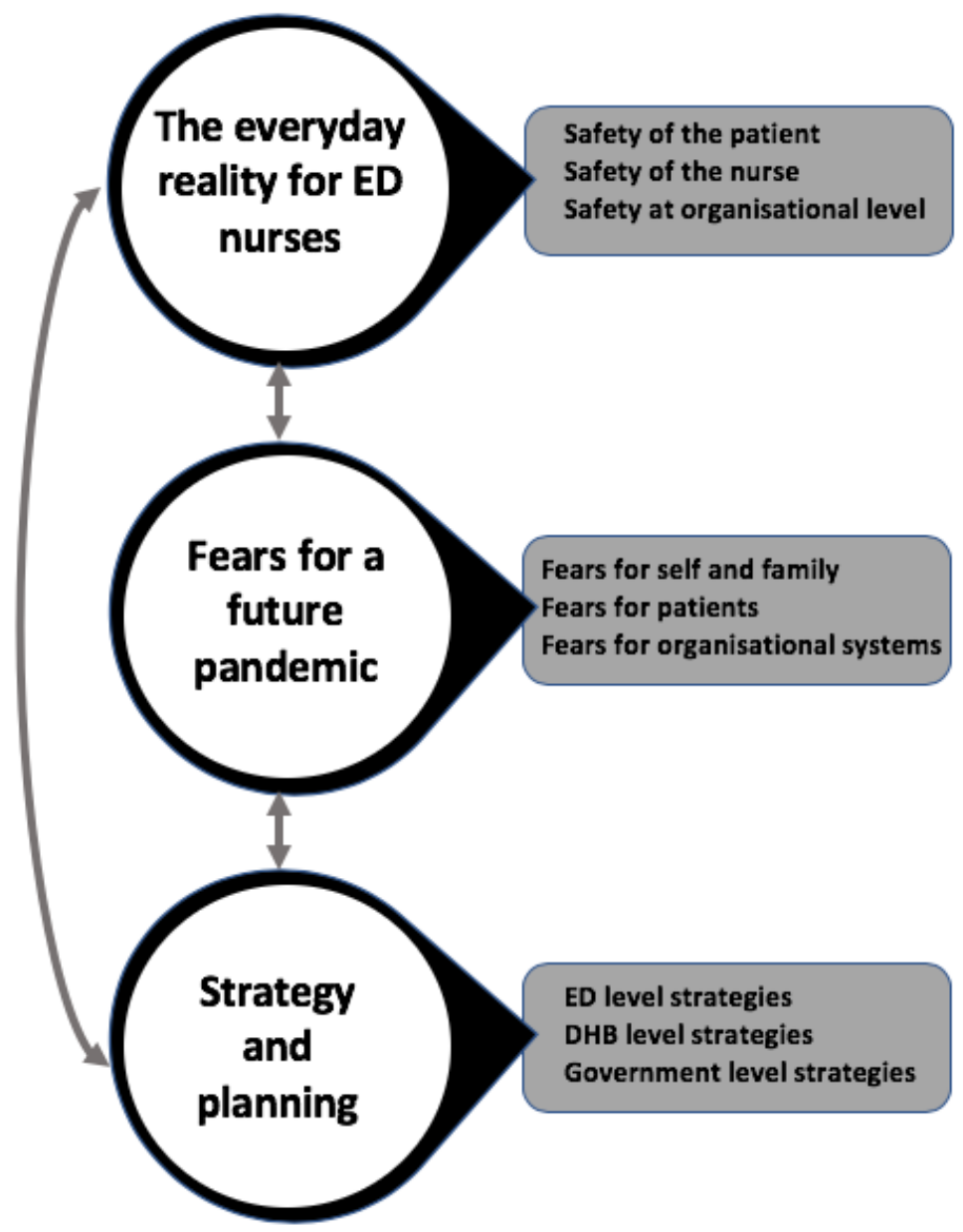

Figure 2: ED nurses' perspectives on existing and future issues regarding pandemic planning in NZ 


\subsection{Participant demographics}

Sixteen registered nurses participated in this study, 10 from site one and six from site two. Of the 16, 13 were female and three were male. All participants identified as European, but their country of origin varied and included some from NZ, Britain, and Australia. Nine participants were in designated senior ED nurse roles, including Associate Charge Nurse Manager, Clinical Nurse Educator, Patient Flow Coordinator and Clinical Nurse Specialist. The length of time as an ED nurse ranged from four to 20 years, with a mean of 11 years in the specialty, and their experience as a triage nurse ranged from 6 months to 20 years, with a mean of 9 years. Seven of the 16 participants were the primary caregiver for dependent family members.

All participants were able to answer questions on all five areas of the interview schedule, often drawing on their personal and professional experiences to address a question. Whilst each participant discussed unique stresses in their individual experience, the common theme was that safety was a problem in everyday ED practice, and would become a significantly larger issue in the context of an influenza pandemic. How ED nurses balance competing safety priorities for both patients and staff in their everyday practice, and their ability to continue to do so during a pandemic, influenced the strategies that they considered necessary for future pandemic management plans.

The interviews averaged 39 minutes (range 25 to 66 minutes). All interviews were audio recorded and transcribed by the researcher, and have been de-identified to maintain confidentiality. Each ED nurse is identified as 'participant' and was assigned a number from 1 through 16 based on interview order. As there were no thematic differences in the findings between the two sites, they are presented collectively.

\subsection{The everyday reality for ED nurses}

This theme provides context around the safety problems that exist within ED nursing practice today, and an understanding of how the decision-making and care practices of nurses are significantly influenced by how patient, staff and organisational risks are 
managed. The common issues identified by the participants related to the safety of the patient with suspected and confirmed influenza, as well as patients who may be at risk from exposure to an influenza patient and are presented in the sub-theme 'safety of the patient'. Participants explained nurses were also at risk of contracting influenza when undiagnosed patients were being cared for in the ED, and this increased risk is outlined in the sub-theme 'safety of the nurse'. Organisational processes within the hospital were seen as an additional area of risk for patients and nurses working in the ED during an influenza pandemic and are included in the subtheme 'safety at organisational level'.

\subsubsection{Safety of the patient}

ED nurses work to preserve and protect the health and lives of those who seek emergency care, but are required to do so in an environment that is often overcrowded, underresourced and unsafe. Workload, staffing and resourcing issues cause nurses concern for the safety of their patients. Care rationing, corridor patients, overcrowding, lack of treatment space and containing the spread of infectious disease amongst patients were identified by participants as the main risks to patient safety.

Care rationing occurs when nurses are so time-poor they are required to prioritise lifesaving tasks over other nursing cares. This was identified as common practice, especially during winter, when ED presentation numbers increased and influenza infection rates are at their highest; "if the workload is too great then some of those minor flu-like illnesses don't get the treatment they should. They don't get obs [vital sign recordings] done or anything" (Participant 16). Participants variously described these situations as "really terrible" (Participant 8), "awful, absolutely awful" (Participant 11) and "one of the lowest points of being an emergency nurse" (Participant 2). Many shared detailed accounts of shifts where they were required to care ration, resulting in patients being in unsafe situations.

I have had two high-dependency patients...I had another confused patient and two other patients, one who was in an isolation room so I just didn't see them....That was definitely a time where I was thinking 
'this is not okay' because people's safety was being compromised, my registration was being compromised and this is not the care that they [patients] deserve. Participant 9

Overcrowding in EDs is a common problem in NZ and around the world, and frequently necessitates patients being in corridors rather than designated treating spaces. Participants identified corridor patients as a significant safety concern, due to the lack of appropriate monitoring and nursing care they recieve.

I've seen patients having CPR [cardio-pulmonary resuscitation] in the corridor and then going into resus [resuscitation room]. I've seen patients $x$-rayed when they've not been assessed and then been found to be heavily pregnant. I've seen patients who have not had vitals [signs recordings] for hours on end because the nurses have just been too busy....There's a lot of near-misses definitely within the corridors and we know that...research states that patients have got higher mortality rates from being in the ED corridors. Participant 15

Overcrowding also results in patients sitting in close proximity for long periods of time in waiting rooms and clinical areas. Participants highlighted that they were concerned about some patient groups during the influenza season because of their heightened susceptibility to infection.

We have lots of oncology patients, haematology patients, young babies, neonates, everyone is mixed within one [ED] department. And these babies are not vaccinated, and we have our immunocompromised patients and they're just all hanging about like it's a bus stop, it's not ideal. Participant 6

Critically unwell patients with influenza can also be placed in close proximity to other patients due to the design layout of the resuscitation rooms at both EDs; "It becomes more tricky when you've got someone who is potentially infectious who is really really poorly and so their clinical need almost outweighs their need for isolation" (Participant 14). Both sites have an identical resuscitation layout, with three adjoining rooms separated by partial walls. The use of a resuscitation room to care for a patient with 
influenza puts patients in all resuscituation rooms at risk, due to the difficulty of maintaining isolation precautions.

Having somebody that is that sick in resus [resuscitation room] and knowing it's not a complete isolation room but next door having an equally sick patient that is probably at high risk now because they've been next door to somebody who needs to be droplet isolated did not leave me with satisfaction. Participant 15

In daily practice, ED nurses regularly make patient care decisions that have a direct impact on patient safety. These nursing decisions involve nurses trying doing the greatest good for the greatest number of patients working within resource constraints, including a lack of staff and space. Participants argued these safety issues would become more frequent and significant during an influenza pandemic due to the increased demands on the healthcare system.

\subsubsection{Safety of the nurse}

The safety problems that exist within the ED environment also affect members of the healthcare team. Nurses experience unsafe situations routinely within their practice, including exposure to the influenza virus, attendance at work whilst unwell, underutilisation of personal protective equipment $[\mathrm{PPE}]$ and difficulty maintaining isolation precautions within the constraints of the physical ED environment.

ED nurses care for undiagnosed patients and because of this, they are frequently exposed to infectious diseases, including influenza. Although only six participants had previously worked in ED during an influenza pandemic, all 16 participants described situations where they had been unknowingly exposed to an infectious airborne disease in their practice. In these situations, participants described feelings of frustration and sometimes anger at being put at risk, with one participant commenting that "it does make you feel a little undervalued at times by the Ministry of Health [MOH]" (Participant 9). Despite these feelings, some participants expressed the view that occupational exposure to infectious disease was a normal part of their job; "It's a 
hazard of being an ED nurse really" (Participant 3). Some participants described questioning their clinical judgement and blaming themselves for not identifying the disease fast enough, and were fearful of the consequences to their own health.

I think that just makes every nurse feel sick and think 'oh my god I'm gonna get sick or have I caught it? or have I done the right precautions that I could have put in place?'...sometimes there can be a lot of selfblame around that like why didn't I pick that up? Participant 1

For those participants who worked in ED during the 2009 H1N1 influenza pandemic, being exposed to the influenza virus in their practice was a common occurrence.

I remember looking after a patient with swine [H1N1] flu but we didn't know they had swine flu and they were tubed [artificial airway] and I remember suctioning them [removing respiratory secretions from a patient's airway] and pulling out the suction and just had my face-mask on and she coughed and it was like swine flu everywhere. Participant 12

The consequences of influenza exposure on nurses can be deadly, as two participants shared their distress about a nursing colleague who contracted H1N1 influenza from a patient and subsequntly died.

She [the colleague] was a known asthmatic and she got the flu from a patient and died. It was horrifying because it was someone I knew well, and she had a little baby and so it was not nice, it was a horrible, horrible thing. Participant 10

Despite clear protocols concerning staff sickness and participants' frustration at being exposed to infectious disease in their workplace, all participants described knowingly attending work sick (known as presenteeism) with respiratory or viral illnesses and being aware that this put patients and colleagues at risk; "If I have like a cold or something, which I'm probably infectious...I think I can just harden up and get on with it then I will" (Participant 2). Reasons for presenteeism included: short staffing "knowing you're already short staffed you do consider whether you're sick enough to stay at home or not" (Participant 1); guilt - "you feel like you're letting everyone else down or that they [colleagues] think you're lying and just not wanting to be there" 
(Participant 3); and a lack of paid sick leave - "People do go to work when they are unwell because they don't have the sick days or they need the money" (Participant 9). When making the decision to call in sick, participants described having to justify the call to themselves, and manage guilt, which affected their ability to recuperate.

The use of personal protective equipment is an international standard for infection control in clinical practice and mandated at both sites. Participants were aware of the expectation to use PPE, but acknowledged they sometimes did not employ these measures in their practice. The most common reason for not utilising PPE was feeling time pressured due to their busy workload, or prioritising the urgent care needs of the patient.

Some stuff [infection control measures/use of PPE] isn't even thought about because you're dealing with something else that is more important and pressing when you're dealing with the patient so that infection control side of things sometimes gets left to the wayside. Participant 13

Even when participants wanted to utilise appropriate PPE, a barrier identified was conflict amongst nursing colleagues, or between nursing and medical staff, when others chose not to use PPE.

I've had cases where I've wanted to put them [patients] in isolation or get people [other staff] to gown up and people have either laughed at you or said 'no they don't need it, we don't need to implement infection control at all' and then you find out that they did and you're just like arrhh...so there's a bit of, argy-bargey, even with doctors and other nurses. Participant 13

The underutilisation of PPE was multifaceted, with participants describing various reasons why this occurred, including "it's an inconvenience I think as well and gets in the way of nursing care" (Participant 13), and "some people are bloody-minded, and seem to think that they are impervious to infections" (Participant 14). All participants acknowledged the difficulty of maintaining proper PPE in the busy and time-pressured ED environment, and all identified instances where PPE was knowingly not utilised 
when it should have been; "if you're racing around because people are sick you often will take shortcuts to get things to happen quicker" (Participant 8).

Best practice in the ED is the use of single rooms for patients presenting with influenza, and for staff to wear masks, gowns and gloves during patient care. Site one had two designated negative-pressure isolation rooms, and site two had one. Both sites also had few other rooms that could be closed off for droplet isolation. Participants identified the lack of isolation rooms as a significant challenge during the influenza season; "we don't have enough isolation rooms so everyone is just cohorted [grouped] in the waiting room to be honest" (Participant 16). When the isolation room was in use for an influenza patient, participants from site two held additional concerns about the location of this room.

It's far away from where everybody is, and you can't hear what's going on and you can't hear any of the overhead calls in isolation. It's not immediately visible....people don't nurse in there if someone is critically unwell because they don't feel comfortable. Participant 13

Despite these layout challenges, participants acknowledged that caring for influenza patients within the dedicated isolation rooms helped to keep them safe from being exposed to infectious disease. This was not the case when influenza patients were so unwell they needed to be managed in the resuscitation rooms which, because of their larger size and additional equipment, are not designed with isolation precautions. Influenza patients often required respiratory support interventions which aerosilised their respiratory secretions onto the staff caring for them "if you've got someone with influenza on BIPAP or CPAP or nebulisers [types of respiratory support delivered via face mask] then it will become an airborne illness and that potentially puts...more staff at risk" (Participant 5).

Some influenza patients in the resuscitation rooms are so critically unwell they require intubation to assist with their breathing. In ED, the time between the decision to intubate and the procedure can be one to two minutes. Despite intubation of an influenza patient necessitating close contact with infectious respiratory secretions, 
participants shared it was uncommon for ED nurses to wear PPE during the procedure. One senior nurse felt that nurses put themselves at risk of exposure automatically, because they are used to responding quickly to stabilise patients in time-critical situations and saving a life is prioritised above considerations for their own safety; "It's incredibly rare that the nurses would think to put PPE on during intubation. They should but they don't because they're incredibly focussed on the patient and their needs but not necessarily focussed on themselves and their safety" (Participant 14).

ED nurses work in an environment where their safety can be compromised daily in various ways. This can be through the actions of others, as well as through their own decision-making, whether they are aware of this or not. The decision to prioritise their own safety can come at the expense of patient care because of the high workload and challenges unique to the ED environment.

\subsubsection{Safety at organisation level}

Organisational factors, often considered to be outside of the direct control of nurses, have an impact on the safety of ED nurses and patients in the day-to-day running of the ED, as well as during an influenza pandemic. These include workload pressures, environmental limitations, barriers to patient flow from ED into the ward and hospital planning and preparedness.

As the population grows, the increased workload for nurses working in EDs has been significant and participants at both sites spoke of ED being left to cope on its own when overcrowded. This lack of support caused frustration and anger amongst participants, who felt "Really unsupported...like no-one cares because in ED we always just deal with it" (Participant 2). The increased patient numbers during an influenza pandemic would create further strain, as participants identified that their EDs were physically too small to manage the current numbers of patients presenting for care with seasonal influenza, and their complex isolation needs. 
It can be pretty hideous.... there would be a lot of people in isolation and then you can't pull out them...so the bed flow of the department can get extremely difficult with a lot of blockage. Participant 15

Working within the constraints of hospitals where demand outweighs resourced acute bed spaces was identified as a frequent challenge by participants. Slow or reduced flow of patients into hospital wards resulted in ED nurses having to balance caring for both new patients presenting for urgent care, as well as patients waiting for ward beds.

Our hospital is too small and its always bed-blocked so unfortunately we have admitted patients sitting in rooms that should be used for...getting our emergency workload through the doors. Participant 2

Compounding the overcrowding was the lack of isolation rooms available within admitting wards. This was a frequent source of delays during influenza season where patients remain in ED, often in non-isolation cubicles, corridors or the waiting room, for hours until an isolation bed is free; "I think we run into trouble because the hospital doesn't run quick enough for us and there aren't enough beds available for people who require isolation... we don't have much space so we get bed-blocked so quickly" (Participant 6).

Participants who worked during the H1N1 pandemic discussed how these same patient safety issues, particularly bed flow problems and overcrowding, were a problem at that time.

The department would just fill up, nobody would move anywhere, the requirements before they [patients] went anywhere in the hospital were astronomical and then when they were in hospital, they were there for days on end. The medical wards...had all these isolation patients which meant they were snowed under which meant bed-block for us. The department would just become gridlocked. Participant 7

The decontamination of clinical areas used to care for influenza patients is an important process in the management of disease transmission within the ED and wider organisation. At site one, cubicles used to care for an infectious patient were 
wiped down with disinfectant before being used for another patient; "you can do an isolation wipe down clean quite quickly, probably within about four minutes and its done" (Participant 5). However, at site two, decontamination of cubicles involved nurses performing an initial wipe down and a cleaner performing a more thorough clean; "we've stripped the linen, we've cleaned whatever we need to clean in terms of equipment and then the cleaner will come and steam-clean the floor and steam-clean the trolleys" (Participant 14). All participants acknowledged that current decontamination cleaning guidelines were confusing and decontamination practices were either insufficient to stop the transmission of disease or too slow. Equipment such as vital sign monitors and electrocardiograph machines that are shared between patient rooms were identified as a risk as "equipment isn't always wiped down as well as it should be" (Participant 7). When there are limited or no cleaning staff available after-hours, rooms were out of commission for several hours, which also impacted on patient flow into the ED.

In summary, this theme provides an understanding of how everyday practice in the ED is driven by issues around safety, with participants discussing the difficulty of maintaining both patient and staff safety within the context of resourcing and organisational system constraints. Participants constantly struggle to manage the many competing safety problems, and when this balancing act fails, are forced to find new ways of stretching existing resources or compromising their own safety in order to provide safe care to their patients. The safety challenges that ED nurses experience in their everyday practice shaped their fears for what could happen in the future if an influenza pandemic were to occur in New Zealand.

\subsection{Fears for a future pandemic}

Participants feared failing to balance personal, professional and organisation needs during a future influenza pandemic, and their fears were influenced by the existing safety issues they encountered in their everyday practice. Their experience of working amongst these issues drove their fears about what could happen to themselves, their families, their patients and the health system in NZ should a pandemic occur. 
Participants also feared a lack of action at organisation level to prevent their concerns from becoming reality, resulting in anger and frustration being directed at DHBs and the $\mathrm{MOH}$.

\subsubsection{Fears for self and family}

Participants recognised that the provision of healthcare during an influenza pandemic is a risk to their own personal safety. Despite this recognition, when participants were asked directly about the risk to themselves during an influenza pandemic, the majority of responses were first and foremost concerned about the safety of their families and the risk of spreading influenza to their loved ones. This was especially prominent if participants were the primary caregivers to dependent children.

It's more about taking that stuff home to my family... like as nurses, we know the risks when we come to work and we do take some risk but when you do put that back onto someone's family then I don't think that's appropriate. Participant 12

Participants discussed the challenge of managing their dual roles as a personal caregiver to family and as a professional caregiver to sick patients, and how making the choice to remain at home or to attend work during a pandemic would be difficult. It would be hard because there's a real pull when you get that text message saying they're really short and you really want to help but you know that you can't and you have to look after your own family.... I think like with anything it's the caregiver that ends up getting sick because you always push yourself to work harder and look after everybody and I think we all fatigue ourselves and we look after ourselves last. Participant 13

When the risk to their own health was discussed, participants worried about the risk of contracting influenza through caring directly for sick patients; "Well I guess the major risk is that I'm going to get influenza and...influenza kills" (Participant 1). Participants who had experience caring for patients who had died from influenza, particularly those patients who were of a similar age to the nurse, were upfront about 
how this contributed to their fear of getting sick; "You know last year we had people die from flu. They were young, like in their 30s and 40s...so that was a bit horrifying because they were people who I wouldn't think would die of something at that age" (Participant 10).

Participants were conflicted as to whether they would continue to attend work during a pandemic due to fear for their personal safety, with several indicating they may reconsider working because of fear; "I don't know if I would continue working if it was becoming a health issue to myself" (Participant 16). Significantly, of the participants who did discuss the possibility of not working during a pandemic, all had previous experience in nursing during the H1N1 influenza pandemic.

Participants also highlighted the possible effect influenza could have on participants financially. These concerns were focussed around the limited amount of sick leave available to staff, and some worried that they would run out of sick leave and would have to take unpaid leave if they became unwell; "the fact is that we [my family] would struggle financially if I had to take time [unpaid leave] off' (Participant 11).

\subsubsection{Fears for patients}

Participants fear that the existing safety problems centered around workload, staffing and resourcing constrants in ED would increase exponentially during an influenza pandemic. Nurses feared being unable to mitigate the risks that patients will face by attending ED for care during a pandemic, failing to prevent the spread of disease amongst ED patients and provide nursing care for those who are already sick.

Drawing from their experience, all participants expressed similar beliefs that their ED would not manage the increase in patient numbers during a pandemic unless significant planning was put in place. Participants feared there would be negative impact on patients if the workload became unmanageable.

We always just seem to cope but at the expense of so many other things so you know, if we're expected to manage a massive surge of patients 
with flu then somewhere along the line, something is going to break. Participant 8

This included fears that the ED would run out of nursing staff to adequately care for patients and that there would be shortages of essential life-saving equipment.

From a resource point of view I think the nurses would get saturated quite quickly and I think our resources would get saturated quite quickly.... Have we got enough BiPAP and CPAP and Hamilton machines [respiratory ventilation support equipment] if they then are that sick? Certainly in the mass casualty I did two years ago we ran out of ventilators. Participant 15

Participants spoke of how the seriousness of seasonal influenza was often minimised because it is challenging to diagnose and difficult to balance the isolation requirements of suspected patients against the needs of others. They expressed fears that this would lead to a slow response during the initial stages of an influenza pandemic, putting other patients at risk; "we are just complacent, it should be guilty until proven innocent like wear appropriate gear, wear the PPE or put them in isolation so nobody else gets sick, like, why do we down play it?" (Participant 3). Minimising the threat of influenza concerned a senior participant, who worried that staff would not identify the infectious disease early enough, causing delays in treatment that could contribute to the death of patients.

I think there is potentially a bit of a common fallacy that flu isn't that bad, that it's just like a bad cold, whereas last season we saw people who were really really seriously affected by it who were sedated and intubated and subsequently died... I think the staff were aware of that and the risk for them is not picking up on the signs and symptoms quickly enough and not triaging them at a high enough category to get these people in quickly. Participant 14

Based on the challenges ED nurses already face managing the isolation needs of seasonal influenza patients, many participants feared that their EDs would be physically unable to accommodate the isolation requirements of every influenza 
patient during a pandemic, or that isolating influenza patients would come at the expense of other patients' monitoring and care needs.

I think we would really struggle because if we have to isolate patients with the flu then that potentially would mean patients who need telemetry monitoring are in the corridor or, you know, the frail. Participant 11

Participants held additional fears that any patient placed into the ED corridor would then be at high risk of contracting influenza from patients with the virus as there are no walls or curtains in the corridors to protect these patients from the spread of disease.

The endless parade of people walking past. We all know about community-acquired infections. According to the literature you can develop an infection just by walking past someone so if it can happen out there I'm sure it can happen in a slightly more sealed area like ED. Participant 7

Whilst the spread of disease directly from patient to patient was the main concern spoken about, participants were aware of other vectors for disease transmission, including from the ED environment due to inadequate decontamination of rooms and equipment, and ED staff themselves acting as a disease vector; "I think there would be increased risk to other patients because I don't think our protocols would stand up to having a huge influx so we'd be more likely to spread it" (Participant 8). During an influenza pandemic, EDs would be required to continue to care for patients with injuries or illnesses unrelated to influenza, and participants feared these patients would be vulnerable to catching influenza, adding to their morbidity burden.

I think the people who present with flu will be treated and given what they need but I just don't think we're going to prevent the other patients in the ED that are actually unwell, especially the elderly or the child with the fracture next door, from being exposed. Participant 8

Whilst the identification and isolation of influenza would present one difficulty during a pandemic, ensuring staff are prepared to care for those with the disease was 
acknowledged as another potential challenge. Some participants expressed they may be less inclined to provide non-essential interventions to influenza patients due to their fear of contracting the disease; "I would probably, in a way, do less interventions because you wouldn't want to touch that person and touch this person" (Participant 16). This attitude was at odds with other participants, who felt they would still have a duty of care to their patients during a pandemic.

It never makes you feel great having to not do something um and it doesn't make me feel like I'm providing the best care that I can for my patient...it definitely doesn't make you feel good inside and it doesn't kind of sit with my morals and my values and my ethics around nursing. Participant 1

The provision of health services during a pandemic will also be greatly impacted by the $\mathrm{MOH}$ pandemic plan, which mandates that less acute influenza patients be triaged away from EDs to community-based services. The concept of triaging influenza patients away from the ED was a contentious issue and a source of fear for all participants, who worried that they would make the wrong decision by not recognising the truly sick or those with the potential to deteriorate.

We're going to feel for their pulses and we're going to assess what their breathing is like and they might look okay at that point in time but... what if the patient goes away and deteriorates and that ends up being on your head. Participant 1

Some participants recalled serious or sentinel events that had occured when patients had been triaged away from ED by their colleagues, which contributed to their reluctance around the process. Other participants with direct experience making an incorrect decision at triage were unwilling to triage away during a pandemic; "I'd leave that decision up to someone else because l've been wrong in the past" (Participant 4). Participants who had direct experience with negative outcomes from triaging away expressed frustration with the pandemic triage policy, and believed that it should be experienced emergency consultants making the decision and holding overall accountability for complex decision-making. 
The consultants load the gun and then give it to the triage nurses...like 'tell them, tell that one, that one and that one to go away' well no, if you want them to go away you go and tell them yourselves, you go and assess them and take that responsibility, don't put that on the nurses because that's not fair. Participant 14

Although participants emphasised many different areas of risk for patients in ED if an influenza pandemic were to occur, each of the issues highlighted contributed to the overall fear that they would be unable to protect the health and safety of patients in the very environment where they have come for help.

\subsubsection{Fears about organisational systems}

All participants expressed a lack of faith in the ability of both $\mathrm{DHB}$ and $\mathrm{MOH}$ pandemic plans to keep patients and staff safe during an influenza pandemic. Nurses feared that current plans were inadequate to address existing safety issues and would result in more problems for ED nurses to navigate during a pandemic. One senior participant stated that their DHB has a record of making well-intentioned policies without providing the necessary resources or equipment for the policies to be fully implemented as intended.

They put all this stuff on us all the time and they never provide us with anything to kind of like make it work...I wouldn't be surprised if there's a hundred other things they've said that we should be doing that we're just not able to do because they don't meet the basic stuff that we need as a department. Participant 8

Participants described how their DHBs were often slow at updating organisational plans, and that by the time plans were finalised the plans were already out of date. They argued that this meant that their EDs were already left with a shortfall in resourcing to manage current demand, with no ability to safety manage the increase in patients numbers that an influenza pandemic would create.

Every year the numbers are going up and up and up and so the staffing we have for those numbers is based on last year's numbers and we're 
only just catching up... we're a few steps behind, so if a pandemic came on we would be completely stretched and at capacity and pretty paralysed. Participant 13

Participants who worked in ED during the H1N1 pandemic reflected upon the lack of planning and preparation that occured; "I can't actually remember any training and I looked after a couple of swine flu [H1N1] patients and I didn't feel prepared" (Participant 12). Fears about poor management during a future influenza pandemic were widespread amongst participants. Difficulty implementing organisation plans for other infectious diseases also contributed to these concerns.

This is the sort of thing that makes me very very very concerned about pandemics because we had a patient that came off the plane that was supposedly a potential Ebola [contagious viral haemorrhagic fever]...there was a protocol put in place where the patient was not to linger in $E D$, they were to go straight up to one of the isolation beds. The first patient arrived and sat in an ambulance in the ambulance bay for four and a half hours before anyone could agree or did anything about it. Participant 7

Previous experience contributed to a lack of trust amongst these participants in the existing DHB pandemic plans, and this lack of trust extended to government planning. They're [Ministry of Health] lying to themselves, we're not well-equipped at all. Well-equipped means that there's an extra unit or area you can open which you can isolate in, well-equipped means there's antechambers, well-equipped means there's stuff in cupboards, a bit like our major disaster cupboard, that is for an influenza pandemic or a pandemic of some sort, so there's aprons, gowns, masks aplenty so we won't run out on day three. Participant 5

Participants also argued that the current government plans were likely written by people who do not have a background in clinical ED practice and hence, were concerned that these plans would lack measures specific to the ED setting. 
This theme highlights that fear was the dominant focal point when participants were asked about a future influenza pandemic. Participants feared for patients, themselves and their families, and no participants expressed confidence that their ED, DHB or the government would be successful in managing the increased patient load and complex needs should a pandemic occur. There was resentment amongst participants towards organisational leadership teams and $\mathrm{MOH}$ officials, whom they considered should be doing more to ensure that the healthcare system is prepared for a pandemic. Those participants with experience of ED nursing during the 2009 H1N1 pandemic were especially critical of exisiting pandemic planning, describing various safety problems that have yet to be addressed a decade later.

\subsection{Strategy and planning}

This theme provides details of the strategies that participants would like to see implemented at ED, hospital and organisational levels, for the successful management of an influenza pandemic. Participants wanted changes made at all stages of the patient's journey through the ED. They wanted their DHBs to be prepared to invest in expanding hospital capacity and staff wellbeing initiatives. Government investment in mandatory influenza vaccinations for healthcare workers, funded vaccinations for the families of ED staff, and improved public health infomation were other strategies participants wanted to see.

\subsubsection{ED level strategies}

The most important strategy participants want implemented is provisions for an increase in nursing and medical staffing to cope with the expected increase in the number of patients seeking ED care. Extra staff would be needed "to transfer patients to wards...triage nurses to help with stuff out the front [waiting area], lots of senior medical officers about to try and move things on" (Participant 7). The paying of overtime rates to staff to come in on rostered days off to ensure the ED is fully staffed, and the availability of on-call staff to assist when the ED was overloaded were proposed. One senior participant identified the lack of ED-trained casual pool staff to 
fill roster gaps, and argued that investing resources in these nurses now would be rewarded if a pandemic were to occur.

I think our casual nurse base needs to be improved... we don't put any resources into the casual pool. There's no education for them and no real incentive to work casual... we need to be able to put on more staff, not just for flu but for any crisis that happens in ED. Participant 12

Due to the increased patient numbers expected, participants argued for a change in the ED model of care during a pandemic. Participants stated that staff should prioritise emergency interventions to stabilise and treat as many patients as possible, leaving some non-urgent nursing cares to be met once the patient reaches their inpatient ward.

I guess you'd change from - from being a bit more attention to detail driven kind of nursing to just critical cares, like emergency interventions only. Like make sure they're alive, breathing, circulating and they're going to for the next thirty minutes and then on to the next one and on to the next one. Participant 4

Triage was another area where participants expressed significant changes should be put in place for a future influenza pandemic. The current set up of triage involves ED nurses and administration staff seated at desks facing the waiting room and requires direct contact with new patients to establish their presenting complaint. This means pandemic patients could easily spread influenza before staff recognised the need for infection control precautions, and participants wanted to see this process changed.

I think that I would be running triage in a different way, I think the admin would be a bit further back with a mask on and people would come in and write on a clipboard and that would get handed over before they go sit back down. Participant 5

Despite an organisational plan that states that influenza patients should be grouped and separated from non-influenza patients in the waiting room, there are no guidelines on how this is to be achieved. Participants considered this inadequate for two reasons. Firstly, both EDs have a single waiting room that is not easily divisible to 
create separation between patients. Suggestions to manage this included utilising the adjoining children's waiting area for influenza patients, or using glass barriers to demarcate the separate waiting areas so that patients could still be seen by the triage nurse in the case of deterioration. Secondly, the identification of influenza at triage which is necessary to appropriately stream patients into separate waiting areas was identified as challenging by participants, due to the various ways influenza can present. There are no guidelines in place at either ED to assist triage nurses with their decisionmaking around the identification of influenza, and participants wanted guidelines to be developed and implemented. Participants also wanted point-of-care influenza testing (POCIT) carried out at triage as a diagnostic tool to help identify those patients with atypical symptoms; "We have the POCIT....so I could do that right off the bat and kind of see and that would tell me fairly definitively whether I would need to have them in isolation or not" (Participant 15).

Guidelines to assist with the triage of suspected influenza patients, and the use of POCIT for rapid diagnosis, would assist triage nurses to identify lower-acuity patients who would be appropriate to triage away from ED, thereby meeting the $\mathrm{MOH}$ pandemic plan.

I think we need some set guidelines, because I'm sure we don't quite do it right sometimes and I'm sure people have turned up to their GP [general practitioner] or After-Hours and they're too sick to be there and get sent straight back. So, having guidelines in place for doing that would be helpful. Participant 6

The concept of influenza pandemic triage guidelines stems from mass casualty triage, where patients undergo rapid assessment of their airway, breathing and circulation and are divided into four care categories: expectant (deceased), immediate, urgent and delayed. ED nurses follow a decision-making flowchart that guides them on which patients should be assigned to each category, and where to place each category group within the ED system; "You would probably have to go into like mass casualty mode or something like that for it to work because we would not cope" (Participant 2). There 
was disagreement amongst participants about which staff held the responsibility for triaging patients away.

As far as I'm concerned, if we're going to turn people away it needs to be from a higher authority than me, a doctor, a medical director, someone in charge of the department or someone with a bit of oomph. Participant 7

Other participants advocated for a teamwork approach to triage decision-making between a nurse and a doctor during an influenza pandemic, where accountability was shared; "during a flu pandemic l'd probably be double checking rather than doing it solo" (Participant 4). This is the model utilised in the mass casualty triage system to make complex triage decisions.

Participants identified that assessing a patient as safe to triage away and providing information on alternative services was time-consuming, and stated an increase in triage staffing would be necessary in an influenza pandemic.

If they're expecting us to be able to triage lots of people away then they need to actually give us the resources...rather than expecting a triage nurse that is trying to triage 20 people at once with multiple things to make that decision. Participant 8

Additionally, participants who had previous experience with sentinel events related to triaging away wanted a safety net provision to protect nurses from Health and Disability Commissioner [HDC] litigation if incorrect triage decisions were made during a pandemic.

I've seen it happen with a colleague... a patient presenting with like a flulike illness, it was suggested to her [patient] that she go see her GP tomorrow, she didn't go see her GP....it was all put into place and documented well but she still had to go through the HDC commission process and that's very stressful and not something that anyone ever wants to go through. Participant 9

Strategies to reduce the transmission of influenza within the ED environment were also considered essential by participants. Once influenza patients have been assessed 
at triage as unwell enough to require care in the $E D$, the separation of influenza and non-influenza patients within the clinical treatment areas was identified as a priority to limit the spread of influenza.

They would need to have a complete area blocked off specifically for flu management. Like people needing isolation requirements where you could actually have all the equipment, like the PPE set up, so literally if you were going to work in that environment then everything is there for you so you're protected. Participant 16

The use of infection control precautions, including hand-washing, is an important part of organisation pandemic plans. However, participants stated that this was not always done well in their workplace, with triage identified as the worst area due to the fastpaced turnover of patients. Participants wanted better access to handwashing facilities to reduce the chance of the triage nurse acting as a vector for influenza between patients.

Another essential part of organisation pandemic plans is the use of PPE for patients and staff. Some senior participants voiced concern for how long the hospital supply of PPE would last in a pandemic, and wanted assurances around adequate supply to ensure "that we've got a hundred boxes spare, that there's masks actually kept for staff as opposed to necessarily making sure it's for patients first" (Participant 5). The decontamination of clinical areas that have been in contact with infectious patients is also a method for reducing the transmission of influenza, however participants reported a lack of guidance in the organisation plans around decontamination. Inadequate decontamination is considered a problem at both sites, but are especially critical at site two where there is no cleaning staff employed overnight and rooms either have to be closed or used for other patients with only minimal decontamination performed by nursing staff. The need for "24 hour cleaning" (Participant 14) is considered crucial by participants to help reduce the spread of disease.

In summary, increasing staffing, changing the ED model of care, pandemic triaging guidelines and limiting the spread of disease within ED were the major strategies that were considered important by participants. 


\subsubsection{DHB level strategies}

Participants wanted their employers, the DHBs, to invest in system-wide changes to manage the increase in patients during an influenza pandemic. The most common change participants at both sites advocated for was the creation of a larger ED. With a lack of ED space identified as an everyday problem, it was suggested that an expansion would create more treatment areas for exisiting patients, as well as allow for more patients to be appropriately isolated and monitored during a pandemic.

Enough space so that we can function as we are and also have a designated area for flu patients because I don't think we can really take away from what we've already got, you know we've already got sick people rolling up so we need to create more space rather than cut down. Participant 4

Requests for more space extended to the hospitals, as admitting wards are frequently at or over capacity, forcing patients to remain in ED for long periods. Participants called for DHBs to develop a pandemic-specific escalation plans that staff in charge of the ED could use to trigger a hospital-wide rapid response to an increase of infectious patients, including redeployment of clinical staff to ED to assist.

I'm unaware of any policy in the hospital that acts as the trigger for them to say... we've had 10 admissions with influenza now and so the ACNM [Associate Charge Nurse Manager] or the CNM [Charge Nurse Manager] of ED can say how many presentations of influenza within a day or two days do I need before we can trigger something in the hospital to help? Participant 15

Another strategy considered by participants to create more treatment areas during a pandemic is the rapid, temporary expansion of ED using existing free areas within the hospital footprint; "There's all that area out the back that is ours where the laundry trollies go....let's put our well patients out there while they're waiting for the wards, you know, it's that thing of we need to start planning" (Participant 5). 
Additionally, the diversion of unwell but stable influenza patients away from ED was identified as a strategy to both manage the limited ED space, and limit the transmission of influenza between patients with and without the virus. Diversion would rely on point-of-care testing for influenza at triage, and patients positive for influenza would be directed to an isolated clinical area for treatment. At site one, the commonest suggestion for diversion was an observation area used currently for shortterm stable patients.

A dedicated isolation unit where you could just fast track patients to where they've all got the flu and you've got them all in the one place and that creates enough space to actually see the ED patients and treat the ED patients. Participant 8

In contrast, at site two, the commonest suggestion was to utilise a nearby medical unit. I would be looking at whether we....turn [medical unit] into an area where you would treat and see these flu patients. So I would assume we would point-of-care test them and if flu positive, go to [medical unit], flu negative they can stay in ED. Participant 10

When infectious diseases are spreading within the community, multiple patients from aged residential care facilities and supported accommodation centres are sent to hospital due to the difficulty managing them within the facility itself. Participants wanted doctors to be sent to these facilities early to assist staff to manage their sick but stable residents without the need to transfer them to the hospital system; "If there's an ability to instead of sending their patients into ED but instead getting someone out to go and assess them there" (Participant 1).

Participants argued strongly that since they were expected to care for infectious patients daily during a pandemic, the DHB should increase sick leave entitlements for staff to avoid nurses being forced to attend work whilst sick. The need for increase in domestic leave entitlements was also expressed, since many nurses are primary caregivers for family dependents.

We don't have separate domestic leave so that eats into your sick leave, so if one of my kids is sick because l've taken a bug home then that eats 
into my sick leave so I end up using annual leave for that stuff. Participant 10

With the knowledge that so many nursing staff are required to care for dependent children, another strategy was for the DHB to offer childcare services for staff, which would allow more ED nurses to attend work to care for the sick.

If I had kids at home it would be really difficult to care for both and to work I think full time and to look after some struggling little kids would be hard. Maybe we can have a creche? A flu creche? Participant 4

Participants wanted DHBs to invest in pandemic planning now, rather than waiting to develop plans during the next influenza pandemic. Both short-term strategies and long-term investments were advocated for to help relieve some of the burden expected by ED during an influenza pandemic.

\subsubsection{Government level strategies}

Participants recognised that some strategies would need to be implemented at government level and felt these should be included in the $\mathrm{MOH}^{\prime}$ s pandemic planning. They wanted free influenza vaccinations extended to the families of frontline healthcare workers and, perhaps controversially, senior participants at both sites felt that influenza vaccinations should be mandatory for all healthcare workers.

Some people [healthcare workers] chose not to [be vaccinated] and I think that they almost shouldn't be given the option to do so because they are then at risk of: a) catching it and b) passing it on to really vulnerable people and I think that part of being a healthcare provider is do no harm. Participant 14

During an influenza pandemic, the $\mathrm{MOH}$ plan involves advising the public to care for sick family members in their own homes where possible and avoid presenting to EDs unless medically necessary. Participants supported this plan, however they stated that public health messages needed to be practical and delivered in a way that all members of the public will understand in order to avoid panic in the community; "Having 
information in the media that is actually driven from healthcare...ensuring that the information getting out is right...getting the message across in the right way" (Participant 1). Participants stated that the $\mathrm{MOH}$ has a major role to play in providing the public with education around influenza self-care before a pandemic occurs. Although there are various reasons why EDs are seeing patient numbers every year, participants highlighted that there was a significant proportion of patients who lacked the knowledge and skills to care for themselves at home when they were unwell with minor illnesses.

I think there does need to be more health promotion and health education for the public because there's a lot that they [patients] can manage before they come to hospital...I think we're [the public] probably too quick now to go to the hospital if there's something wrong with me whereas in many cases we can stay at home and keep the fluids up and isolate ourselves. Participant 15

Another crucial part of the government's pandemic plan is the establishment of community-based assessment centres (CBACs) to treat and discharge stable influenza patients back to their own homes. Participants felt that these CBACs needed to be staffed by experienced senior nursing and medical staff for ED staff to feel that triaging patients away from ED to a CBAC is appropriate.

[If] I know that the nurses and doctors there have been given education and can handle it well then a person that comes with a mild influenzalike illness does not need a hospital, they're not going to need interventions that we offer and having them out of our physical department is better for them and better for the safety of our patients, especially those that aren't there with influenza like illness. Participant 5

However, participants were concerned that it would take time during the initial stages of a pandemic to establish CBACs, and argued for solutions that could be implemented faster, including extending primary healthcare clinic hours and subsidising the cost to see a GP. These strategies would give participants more confidence in triaging patients away knowing that some of the barriers to accessing primary healthcare, namely cost 
and convenience, have been addressed "[An] after hours clinic that costs \$15 or \$25. Then I'd feel a lot more comfortable" (Participant 5).

In summary, this theme presents the strategies that ED nurses argue are necessary for inclusion in future planning for the health system to successfully manage an influenza pandemic. Participants expressed that it is crucial for EDs, DHBs and the government implement these recommendations before an influenza pandemic occurs in the future.

\subsection{Chapter Summary}

This chapter provides insight into the current problems in emergency nursing practice today that directly affect the safety of ED patients and staff, how these problems provide the foundation for the fears ED nurses hold about how they and their workplace would cope during an influenza pandemic, and short and long-term strategies these nurses want to see implemented in future plans to help prevent or limit many significant issues. In the following chapter, the meaning of these findings are discussed in the context of the literature, and recommendations for the application of the findings to the NZ health system are presented. 


\section{CHAPTER FIVE: DISCUSSION}

\subsection{Introduction}

This research aimed to determine the strategies and processes ED nurses consider important to safely manage during an influenza pandemic. Nurses' perspectives on the impact a pandemic could have on NZ EDs, and what impact a pandemic could have on the safety of themselves, their families and ED patients was explored. The key findings of this research are that decisions are made everyday by nurses that affect the safety of both patients who seek care in the ED, and the safety of ED nursing staff. The provision of safe nursing care was constrained by the limitations placed upon nurses by existing organisational systems. The responses from the participants provide an in-depth understanding of the safety problems that exist within current ED practice, and how these issues influence the fears nurses hold about what could happen to patients, nurses' families and themselves during an influenza pandemic.

The challenging everyday reality for ED nurses not only shapes the fears participants hold about future problems, but also provides context behind the strategies the nurses considered are needed to better meet the health needs of NZ's population during an influenza pandemic. These strategies include changes within the set up of their EDs, as well as wider strategies for DHBs and the government to implement to manage and overcome the challenge of a future pandemic. Recommendations from the author and a discussion on the strengths and limitations of the study are also presented.

\subsection{The everyday reality for ED nurses}

Within the ED setting, nurses readily discussed safety problems within their daily practice that affect patients, staff and the hospital as a whole. These safety issues form the basis of the first theme, the everyday reality for ED nurses. The safety issues highlighted by the participants are heavily influenced by the dynamic nature of ED work. Caring for patients with infectious diseases is a common situation in ED, especially during the influenza season. However, the infection control needs of these 
patients must be balanced against the needs of other patients. This can be difficult in the frequently busy and overcrowded ED environment, and nurses are constantly reprioritising their workload to manage or mitigate the complex needs of all of their patients.

\subsubsection{Safety problems through nurse actions}

The placement of patients into corridors is a common occurence in ED. Nurses move patients into corridors as a way to manage overcrowding in a physical workspace that is considered too small for the requirements of the population. Swedish researchers undertook a grounded theory study into the management of waiting times in EDs and found that the placement of patients in corridors during times of overcrowding was intended to improve the flow of patients through the ED. However, when such placements occured nurses felt frustrated and ashamed, and found it difficult to observe deterioration in the health status of the corridor patients (Burström, Starrin, Engström \& Thulesius, 2013). It is significant that this phenomenon occurs during times of overcrowding and high ED and hospital occupancy, as the rate of serious complications is increased for patients admitted through an overcrowded ED (Zhou et al., 2012). ED nurses in my study acknowledged that patients placed in corridors were more likely to receive inadequate care and they held considerable concerns for patient safety. All participants were able to recall incidences of near-misses or actual harm occurring to patients placed in corridors. With overcrowding expected to be exponentially worse during an influenza pandemic, the placement of patients into corridors is likely to be inevitable. Whilst the use of corridors as a treatment space is a tool used by ED nurses to manage the problem of overcrowding and physical space restriction, it subsequently creates another problem in that patients are placed into a riskier area of the ED and receive less nursing care. This creates a paradox, where the use of one method to manage or mitigate risk has the side-effect of creating a different risk for the patient. Further research is necessary to understand the decision-making process ED nurses undertake within the context of this paradox. 
Normal practice within the study EDs is that lower acuity patients are placed in corridors to allow for sicker patients to be monitored in cubicles. During an influenza pandemic most lower acuity patients are likely to have been triaged away from ED, meaning some higher acuity patients could be placed in corridors and any deterioration in their status may not be recognised soon enough by nursing staff, if at all. This presents a significant risk to patient health and safety which has not been acknowledged or addressed in hospital or government level pandemic planning. The influenza pandemic plan of the study site DHB acknowledges patients will be in corridors, but offers no strategies on their specific care $(\square, 2018)$. Research on the use of corridors as treatment spaces has so far focussed on the increased mortality rates and the negative psychosocial effects on patients regarding the lack of dignity and privacy (Foss \& Krogstad, 2000; Richards, van der Linden \& Derlet, 2014). With the practice of placing patients into corridors occurring with regularity at both study EDs, further research must be undertaken to understand the decision-making process ED nurses undertake when placing patients in corridors, and how the increased risk to patient safety identified by nurses can be mitigated, both for everyday practice and during an influenza pandemic.

Another action nurses take that can have safety ramifications for themselves and patients is presenteeism, continuing to attend work whilst unwell. Nursing presenteeism is a recognised phenomenon that has been studied extensively (Aronsson, Gustafsson \& Dallner, 2000; Rainbow \& Steege, 2017). Nurses demonstrate a three to four-fold higher rate of presenteeism than those in the private sector, and this is mainly due to short-staffing, workload demands and a professional identity of nursing self-sacrifice to attend work to care for patients (Rainbow \& Steege, 2017). There is a paucity of ED nursing-specific presenteeism research, and these findings contribute the perspectives and experiences of ED nurses on this phenomenon. The findings of this study are in line with other presenteeism literature, with nurses describing staffing availability as the most significant factor in their decision to not take sick leave when they were unwell. An increase in staffing numbers for pandemics could help to manage nursing roster gaps and assist with the workload, reducing presenteeism rates within ED. 
Additionally, nurses described their limited amount of sick leave as another contributing factor towards their presenteeism. Whilst nursing presenteeism research has focussed on other factors, research within the private sector has demonstrated that having paid sick leave reduces presenteeism rates (Asfaw, Rosa \& Pana-Cryan, 2017). NZ nurses are given 10 days (pro-rated for part-time employees) of paid sick leave (NZNO, 2018). However, with ED nurses known to have a higher risk of occupational exposure to infectious disease, a case could be made to increase sick leave entitlements as a method of reducing presenteeism rates and the loss of productivity and risk to patients that it brings.

Presenteeism to meet staffing shortfalls creates two significant problems that can negatively impact upon ED patients. Firstly, carrying out direct patient care whilst unwell creates a risk that the nurse could spread their illness to patients. During an influenza pandemic, EDs are high-risk areas for hospital-acquired infections, and the presence of staff infected with influenza will further increase this risk (May, Lung \& Harter, 2012). Secondly, fatigued nurses are more likely to make mistakes and put themselves at risk of further illness or injury (Halbesleben, 2010; Rainbow \& Steege, 2017). In his study of nurses and other health professionals, Halbesleben showed a link between exhaustion, unsafe behaviours and workplace injury. With ED nurses working through sickness, it can be inferred that there is a high chance they will be more exhausted than their well colleagues. This places these nurses at risk for workplace injuries, and within the context of a pandemic, a potentially higher chance they will circumvent safety protocols designed to limit their exposure to influenza, increasing their risk of developing the disease. The findings of this research have demonstrated significant contributing factors to presenteeism in ED nursing, a phenomenon which has previously been little researched. Presenteeism has been shown to result in the loss of 1.5 times more working time than absenteeism (Perry, Lamont, Brunero, Gallagher \& Duffield, 2015). By addressing the contributing factors demonstrated within the findings, DHBs could have a safer, more productive ED nursing workforce, more able to care for the complex needs of patients and less likely to contribute to the transmission of disease during an influenza pandemic. 


\subsubsection{Safety problems through nurse omission}

The inadequate use of PPE when caring for influenza patients was identified by ED nurses as a regular occurence. This finding is in line with other ED literature (Liang, Theodoro, Schuur \& Marschall, 2014; Picheansathian, 1995). Infection control is challenging in ED as the assessment of infection risk and decisions around treatment are initially made based on incomplete data and influenced by time and resource limitations (Liang et al., 2014). Workload is the greatest factor in the proper use of hand hygiene and PPE in EDs, and other factors include a lack of time or equipment, the inconvenience of utilising PPE and a lack of understanding of the consequences of not using PPE (Picheansathian, 1995; Westphal, Lancaster \& Park, 2014).

Adherence with infection control precautions has been found to be significantly lower when nurses care for patients within ED corridors, with one study finding adequate droplet precautions were used for only $7 \%$ of influenza-like illness (ILI) patients cared for in ED corridors (May, Lung \& Harter, 2012). These findings are validated by the ED nurses in this study who confirmed that ILI patients can be placed within corridors despite the known increased risk of transmission of influenza to other patients. Additionally, this research highlighted conflict between colleagues who held differing views around PPE usage as a widespread problem that affected compliance. This conflict has not been well studied, but factors considered to contribute to conflict include a lack of shared safety culture, social distancing and obstruction of clear communication created by the physical act of wearing the PPE equipment, and the lower rates of adherence to infection control precautions by medical staff (Erasmus et al., 2010; Liang et al., 2014).

The nurse participants also discussed the difficulty of maintaining PPE in timepressured and clinically urgent situations, such as the need to perform rapid sequence induction and tracheal intubation of patients. This finding is supported by literature which reports that infection control precautions are often overlooked or ignored in life-threatening situations, and that ED nursing and medical staff have poor adherence 
with both mask and glove use when working with patients with ILI (Liang et al., 2014; Turnberg et al., 2008). My research demonstrates that the choice to forgo PPE in favour of prioritising the stabilisation and treatment of a critically unwell influenza patient is a practice some ED nurses employ to ensure that patient care is not compromised when their needs are time-critical. By choosing not to utilise PPE in such situations and thereby exposing themselves to infectious respiratory secretions, ED staff put themselves, their colleagues, their families and other patients at risk of developing influenza. ED nurses shared this as something they feel they 'have to do' in some situations to save the life of their patient, and one senior nurse felt that staff were so focussed on saving their patient that the thought to use PPE would not even be considered. It is apparent that the need to save patient lives at the expense of their own health is so ingrained within ED nursing culture that it has become an automatic reaction. This is a significant new finding that may help explain why ED staff do not choose to apply safety policies that are in place specifically to minimise risk to staff. More research is required to establish how to best address this risky decision-making to prevent negatively impacting on patient safety in time-critical situations.

Across both study sites, policies exist to guide the decontamination of the ED environment after the presence of an influenza patient. However, this research found that these policies were often confusing and unclear, and ED nurses feel that decontamination processes are too slow, causing delays in care for other patients. Circumventing these policies was common, especially attempting to decontaminate treatment spaces themselves rather than to wait for the cleaning staff, often resulting in shared equipment not being cleaned as thoroughly as necessary due to time constraints. This circumvention creates a subsequent safety problem for staff and patients, particularly as the influenza virus can survive on cloth (e.g., patient gowns and bed linen) for up to a week, and on stainless steel surfaces (e.g., patient trollies and overbed tables) for up to two weeks (Thompson \& Bennett, 2017). High-touch patient objects (e.g., chairs, trollies and curtains) are often routinely cleaned within EDs to minimise the risk of these objects acting as fomites (objects that carry infection), but other objects such as computer keyboards, phones and stethescopes, may be infrequently cleaned and are often contaminated with bacteria (Liang et al., 2014; 
Núñez, Moreno, Green \& Villar, 2000). Further exploration is needed to determine if these objects could act as fomites for influenza.

\subsubsection{Workarounds}

Both the actions ED nurses take (e.g., caring for patients in corridors, working through sickness), and the omissions they make (e.g., non-compliance with PPE and decontamination protocols), are examples of circumventing existing safety processes to enable patients to receive the nursing care they require. These behaviours are mechanisms to address workflow issues and operational failures, such as high patient loads and lack of space within the ED, both of which are frequent problems during influenza season. Within the literature, these behaviours are commonly termed 'workarounds', offering solutions "to a range of problems including shortcomings in staffing, equipment and supplies" (Debono et al., 2013, p.2). Workarounds have been widely researched in medical, surgical, paediatrics, maternity and intensive care settings (Debono et al., 2013). However, there is a paucity of research in the ED (Kahol, Vankipuram, Patel \& Smith, 2011; Mentis, Reddy \& Rosson, 2010; Picheansathian, 1995).

Studies have shown that medical and surgical nurses spend at least $10-15 \%$ of their time working around barriers to patient safety and care, and in some cases nurses were found to be utilising workarounds approximately hourly, equating to one in every 15 nurses spending their entire shift working around operational failures (Paparella, 2018; Tucker, Heisler \& Janisse, 2014). Overcrowding and high patient turnover in the ED means nurses there see more patients per shift than their medical and surgical colleagues, which carries the possibility that they are undertaking a higher percentage of workarounds (Debono et al., 2013). Workarounds are more likely to occur when the work environment is busy, priorities are constantly changing and staff are frequently interrupted, which encompasses the frenetic and dynamic nature of ED (Walter, Raban \& Westbrook, 2019). My study found that workarounds occured with regularity in ED, especially during the busy influenza season, and included caring for patients in corridors to manage overcrowding, presenteeism whilst unwell to cope 
with limited staffing resources, non-compliance with PPE due to time and workflow constraints, and circumventing decontamination guidelines to accelerate the availablity of ED cubicles and bed spaces for new patients.

It is significant that various workarounds were identified and discussed by the ED nurses within my study despite workarounds not being the focus of the research. If workarounds are utilised this frequently for the subset of patients presenting with infectious diseases such as influenza, it is possible that workarounds are occuring regularly to manage patients in EDs on a daily basis. Furthermore, my research demonstrates that although the workarounds employed by ED nurses are an attempt to improve patient safety in the busy, understaffed and overcrowded ED environment, the workarounds themselves create patient safety issues. The workarounds described by these ED nurses work as a temporary stop-gap measure to manage an immediate problem, but are then creating subsequent safety problems elsewhere along the line during emergency care without addressing the primary underlying issue of ED underresourcing. This highlights the discord apparent when one problem is solved and another created, resulting in a paradox which has not previously been discussed in the workaround literature.

With limited research available about ED-specific workarounds, the methods utilised by ED nurses in this research could be used to highlight problems not previously recognised or acknowledged at organisational level. This research could also help guide system-wide improvement projects to meet operational shortfalls and enhance patient safety in EDs. By investing in these solutions now, this could help reduce the risk for patients presenting for care in EDs during an influenza pandemic when resourcing issues will be widespread.

\subsection{Fears for a future pandemic}

Fear of an influenza pandemic is the second major finding in this research. ED nurses held both personal and professional fears in such a situation. Personally, the nurses feared for the health and safety of themselves and their families when faced with the 
increased risk of contracting influenza through their work. Professionally, ED nurses are already struggling to meet the complex needs of their patients in ED (Ardagh \& Drew, 2015; ACEM, 2019). This problem will get significantly worse during an influenza pandemic due to the increase in patients, and nurses feared not being able to provide care for all who need it, running out of vital equipment and resources, and having to turn people away who have sought help at ED. They also feared that current pandemic plans will fail to assist with ED's workload and resourcing issues.

\subsubsection{Personal fears}

With nurses already feeling as though they compromise their own safety to meet the complex needs of their patients, ED nurses expressed significant fears for the health and safety of themselves and their families during an influenza pandemic. Current pandemic plans do not have clear provisions for the level of personal and professional risk expected of ED nurses by their own workplace and the general public during a mass event like an influenza pandemic. ED nurses feared conflict between their overlapping personal duty of care to their family, and professional duty of care to their patients.

Demand for healthcare will rise significantly during an influenza pandemic, and nurses will be an essential part of the healthcare system response. The findings show that ED nurses are conflicted between protecting their own personal safety and health, and their feelings of responsibility to patients. ED nurses struggled with the knowledge that influenza can cause critical illness and death, even amongst their own colleagues, and their feelings of responsibility to attend work if an influenza pandemic was widespread. The participants displayed a level of moral distress at these conflicting priorities, and this should be concerning for the healthcare organisations that employ nurses, as moral distress has been directly linked to job satisfaction in nursing (Asgari, Shafipour, Taraghi \& Yazdani-Charati, 2019).

The responsibility that nurses feel to provide care to their patients is closely linked with the concept of 'duty of care', which is the legal, ethical and professional 
obligation for nurses to prevent harm coming to patients, and breach of this duty can result in disciplinary action (Water, Rasmussen, Neufeld, Gerrard \& Ford, 2017). There has been much debate within disaster medicine and ethics literature concerning healthcare professionals duty of care during a mass-casualty incident, versus their personal responsibility to themselves and their families. One perspective revolves around the idea that healthcare workers are the only members of society who hold the necessary skills required to care for pandemic patients, and this increases their obligation to society to provide care (Ruderman et al., 2006). Conversely, Sokol (2006) argued that the term 'duty of care' is too broad, and argued that healthcare workers should not be compelled to work as this broad policy did not recognise that these workers hold multiple roles in a community. These roles include responsibility to their spouse, children and other family members to protect them from harm by minimising their own risk of infection.

ED nurses described the fear of spreading influenza to their family and friends as a major fear when faced with working during an influenza pandemic. They felt that the possibility of bringing a dangerous virus home to their family is an unacceptable risk. Disaster medicine research has argued that nurses assume an increased risk to themselves when they chose a healthcare-based profession (Ruderman et al., 2006). However, whilst the ED nurses did fear for their own health, they were concerned about bringing influenza home and infecting their family, as their family members did not consent to carrying the increased risk that the individual nurses assume through their profession. Part of the MOH's pandemic plan involves keeping lower-acuity influenza patients at home to be cared for by family members. Many nurses in this study hold caregiver roles for dependent children and have personal obligations to ensure the safety of their own family. These nurses argued that the possibility of bringing a dangerous virus home to their family is an unacceptable risk and consider it unfair that this might be expected of them.

There is a concerning lack of guidance around the professional and personal duty of care for nurses in a pandemic situation. Whilst the New Zealand Nurses Organisation (2016) acknowledges that there can be a conflict between professional and personal 
obligations, it believes it is up to the individual to decide how to manage these competing priorities in a pandemic situation. Notably, the $\mathrm{MOH}^{\prime} \mathrm{s}$ national pandemic plan contains no mention of duty of care expectations for nurses. Significantly, the conflict between personal and professional obligations was an important concern for nurses in this research. Stronger guidance is required to define the limits of the professional duty of care during a pandemic. However, such guidance will require consultation amongst healthcare worker groups at national level to determine what limits would be considered acceptable.

\subsubsection{Professional fears}

Under normal circumstances, the universal healthcare system in NZ aims to provide every person with the care they need, when they need it. However, in a mass-casualty situation there is an "acute and unforeseen imbalance between the capacity and resources of the medical profession and the needs of survivors who are injured whose health is threatened" (McCullough, 2010, p. 322). With finite resources available, difficult decisions must be made around the allocation of those resources and it is these decisions, and their impact on patients, that concerns ED nurses during a pandemic.

When demand outweighs resources in an influenza pandemic and it is impossible to manage the needs of every unwell patient, health professionals are required to determine who receives priority treatment, and in what order that treatment is provided. These decisions are based upon the utilitarian principle of doing the greatest good for the greatest number (Greenacre \& Fleshner, 2017). It is predicted that PPE, antiviral medications, ventilators and inpatient admitting beds will likely be inadequate during a pandemic (Verweij, 2008). In 2005, it was estimated that there were approximately 105,000 ventilators in the United States, with 100,000 in use during seasonal influenza. However, during an influenza pandemic, it is likely that some patients who need ventilation will not have access to one due to resourcing issues (Osterholm, 2005). ED nurses in this study held concerns that their EDs would not cope with the number of patients presenting for care nor with their complex needs 
in an influenza pandemic. This concern is based on the projections from the $\mathrm{MOH}$ (2017) that an influenza pandemic could affect approximately 1.9 million people, $40 \%$ of NZ's population, and cause more than 38,000 deaths. With hospital services already operating at near-capacity, nurses would quickly be expected to work in an environment where decisions were needed regarding prioritising finite resources.

Aside from vital equipment, nursing care is also an essential resource that will be limited during an influenza pandemic. Nurses, like all members of society, will be at risk of contracting influenza during a pandemic. This creates a problem as the demand on ED and nursing resources will significantly increase. However, with nurses being vulnerable to becoming sick during a pandemic, nursing numbers may decrease with no ability to fill staffing gaps (Verweij, 2009). Nursing researchers argue that when nurses are required to ration their care to safely manage a large number of sick patients, this "compromise[s] the patient's right to health care, which conflicts with personal and professional values" (Suhonen et al., 2018, p. 26). Holt (2008) discussed the allocation of staffing resources after Hurricane Katrina left New Orleans with a lack of nursing and medical staff to care for acute casualties as well as current inpatients. He recommended that guidelines for resource decision-making be based on patient prognosis, with staffing and hospital beds allocated away from existing inpatients with terminal illnesses or do not resuscitate orders, and instead directed towards acute patients who have a better prognosis. Holt further emphasised the importance of educating the public about these requirements before a mass-casualty event.

Medical ethicists debate how best to manage such a situation, but what is crucial is that in the event the health system is overwhelmed by a pandemic, decisions around which patients would receive inpatient care and resources and which would not need to be open and transparent to the public, to reduce "distrust in professionals, institutions and governments" (Verweij, 2009, p. 161). The ED nurses voiced their distress when they had to ration their nursing care due to overcrowding, and this problem would exponentially increase during a pandemic, negatively impacting on nurses' mental health during an already difficult time. Removing the responsibility for 
making these difficult ethical decisions may assist in negating some of this impact, and should be considered at government level.

The $\mathrm{MOH}$ plan for managing finite ED resources during a pandemic centres around the process of directing influenza patients to a community-based assessment centre (CBAC) to take some of the demand off EDs. CBACs provide assessment, advice and distribution of essential medicines. They do not have any capacity for inpatient admission or observation of unwell patients $(\mathrm{MOH}, 2017)$. The National Health Emergency Plan notes that it is advantageous to locate CBACs near to public hospitals, as "it may be possible to screen all patients presenting at the ED and divert infectious people to the appropriate unit" ( $\mathrm{MOH}, 2008$, p. 12). The study site DHB influenza plan also specifies that it may be necessary for the ED to redirect non-urgent presentations away to community-based care, and that hospital care will be limited to the most seriously ill patients $(\square, 2018)$. It is significant that there is no specific guidance on how ED staff are to screen patients in these scenarios, or how to determine which patients are well enough to be diverted away from ED. Screening patients on arrival to ED to determine clinical urgency is the role of the ED triage nurse, and the potential requirement to divert some lower-acuity patients away from the ED, also known as triaging away, during an influenza pandemic concerned participants in this study (College of Emergency Nurses New Zealand, 2009). Participants felt that triaging away amounted to a refusal of care for patients, which disregards a patient's right to make decisions about their own healthcare. If they have made the decision to present to an ED because they believe they have a health emergency, then triaging them away is a refusal to respect the patient's autonomy and their choice around their own healthcare (Aacharya, Gastmans \& Denier, 2011). Ethics and law researchers propose that the use of prescribed mass-casualty algorithms for triage removes the autonomy of the nurse as well as the patient, by removing any subjectivity in their decisionmaking. By adhering strictly to a guideline, complaints of unfairness or inequity against the individual nurse are reduced (Greenacre \& Fleshner, 2017). The mass-casualty triage system is different from the Australasian Triage Scale used in NZ EDs; it prioritises limited resources in a timely, equitable and efficient manner to do the greatest good for the greatest number (Christian et al., 2006; Greenacre \& Fleshner, 
2017). Using the mass-casualty triage system could still be a contentious issue as patients are used to receiving a high standard of care, however the $\mathrm{MOH}(2017$, p. 15) recognises that "people are more likely to accept difficult decisions if decision-making processes are open and transparent, reasonable, inclusive and responsive, with clear lines of accountability."

Not only did ED nurses feel that triaging away was ethically difficult, but many expressed fears that they would make an incorrect decision and not recognise an influenza patient who was sick enough to require care. Previous research identified that current triage tools are poor predictors of disease morbidity and hospitalisation rates. An American study found that of 106 patients deemed to have 'non-emergency complaints' and therefore considered safe to triage away by ED nursing staff, one third required further treatment including hospitalisation (Lowe et al., 1994). A similar Canadian study of 25,000 ED presentations showed that 316 patients were incorrectly triaged into the lowest acuity category and required hospital admission (Vertesi, 2004). Both studies were retrospective audits, and crucially the authors of the Lowe et al. study stated that it was thought unethical to design a randomised trial where low-acuity patients were triaged away from ED for follow up, hence the decision to use a retrospective study design. If triaging-away is unethical in a relatively controlled research context, then it is significant that DHBs may potentially ask ED nurses to triage-away during an influenza pandemic. The fear of the medical, ethical and legal consequences of making the wrong decision and triaging away a patient who deteriorates or dies is a driving factor in nurses' reluctance to perform the task (Lowe et al., 1994).

Professional responsibility during an influenza pandemic also extends to DHBs and other services who employ nurses. Medical ethics researchers have argued that in exchange for nurses putting themselves at risk by caring for sick patients during a pandemic, their employer must take all practicable steps to minimise risks to the health of their employees, including the provision of PPE, additional support to families if the healthcare worker is required to remain in quarantine, and monetary 
benefits to be paid out in the event of significant injury or death (Godkin \& Markwell, 2003). This is in line with the recommendations of the WHO (2007), which mandates that hospitals and government organisations have a reciprocal responsibility to minimise threats to the personal safety of their nursing and healthcare staff during a pandemic. The WHO also state that healthcare workers should be morally obligated and possibly compelled by law to work as they are considered an 'essential service' in an influenza pandemic, provided their employer has met these reciprocal responsibilities. However, the findings of this study demonstrate that ED nurses expressed a lack of confidence in both their employers and the government to keep them safe during an influenza pandemic. They feel these two organisations are underprepared and under-resourced, and ED nurses and other healthcare professionals would therefore not be morally obligated to work during a pandemic as their employers have failed to meet their reciprocal responsibilities as per WHO recommendations.

The various and significant fears raised by the nurses require further research and consultation with nurses and their professional bodies to determine the most appropriate solutions. The urgency of the need for this research cannot be overstated, because the significance of the dilemmas cannot be ignored and should be dealt with before a pandemic situation arises.

\subsection{Strategy and planning}

Working in a resource-constrained environment with widespread safety issues has not only influenced the fears ED nurses have about a future influenza pandemic, but also the strategies they feel are necessary for the health system to successfully navigate through a pandemic. These strategies range from ED-level recommendations, including pandemic triage tools, point-of-care influenza testing at triage, segregation of waiting rooms and increased staffing, to hospital-level recommendations such as investment in a larger ED and hospital overall, rapid, temporary methods of increasing ED space, and the use of incentives to come to work. Furthermore, ED nurses felt that change was needed at government level, and strategies at this level include the 
introduction of mandatory vaccinations for healthcare workers, dedication to public health promotion and managing the flow of information to the public, and legal protection for triaging patients away from ED.

\subsubsection{ED level strategies}

Triage is an area that all participants felt would be dangerous to work in during an influenza pandemic. The nurses feared triaging patients away from ED inappropriately as influenza can be difficult to recognise and patients can quickly deteriorate (Chen et al., 2004). They were divided about the best strategy to manage this situation with some nurses wanting clear, concise guidelines to assist with their triage decisionmaking, and others arguing that their medical colleagues should assist with the triage process.

Both of these strategies could be met if an influenza pandemic triage tool was developed, based on the current colour-coded, four-category triage system for mass casualty incidents. This system provides a flow-chart for nurses to follow, using physiological parameters to guide decision making, and is also taught to medical staff, allowing their inclusion in the process (Ardagh et al., 2012). However, the system is based on the rapid control of airway, breathing and circulation and assessment of traumatic injuries, rather than illness, and would need to be adapted to be useful in an influenza pandemic. During the H1N1 pandemic, various tools were used worldwide to triage influenza presentations, including the CURB-65 pneumonia score and the Pandemic Modified Early Warning Score. However, both showed poor accuracy for predicting morbidity and mortality because they did not take into account obesity, pregnancy and significant co-morbidities known to correlate with a worse prognosis for pandemic influenza (Goodacre, Irving, Wilson, Beever \& Challen, 2015). Further study is required to develop and model a triage tool for use in an influenza pandemic that predicts a patient's risk of critical illness or death, with the ability to more accurately detect low-risk patients who would be suitable to be triaged away from the ED. This triage tool needs to be appropriate for use in the NZ population, 
which may have different co-morbidities and health outcomes that influence risk prediction than other countries.

Nurses discussed the difficulty of identifying influenza at triage, contributing to their fear around triaging patients away. The nurses called for the use of rapid diagnostic influenza testing at triage, and this strategy is supported by research that notes "diagnosing influenza on the basis of only clinical symptoms is difficult because manifestations vary and are nonspecific" (Merckx et al., 2017, p. 395). Both study site EDs have access to a point-of-care testing instrument that takes approximately 15 minutes to run a nucleic acid amplification test (NAAT) to detect influenza. However, its use is restricted to patients awaiting admission to an inpatient bed to determine the need for an isolation room. This instrument has tested high for both sensitivity (ability to detect a true positive result) and specificity (ability to detect a true negative result) compared to the current gold-standard polymerase chain reaction (PCR) testing, which takes several hours (Bell \& Selvarangan, 2014). There are two benefits to the rapid influenza testing at triage strategy. Firstly, it would to help identify loweracuity influenza patients that could be suitable for triaging away to community-based services. Secondly, it would help to rapidly identify those patients at risk for spreading influenza to staff and other patients within the ED environment.

The positive identification of patients with influenza would allow for these patients to be cohorted and isolated in a separate area of the ED. Strong evidence exists for grouping unwell patients to avoid overlap between influenza and non-influenza patients to prevent the spread of the disease in both inpatient and outpatient settings. This should be strongly considered for ED waiting rooms, which will be required to manage high volumes of influenza patients on top of their regular emergency workload (Uyeki et al., 2019). The waiting room segregation of influenza and noninfluenza patients is included in the study site pandemic plan, however neither ED has the ability to physically segregate their waiting rooms, and methods to do so need to be investigated. 
Another strategy discussed by the nurses was the need for more staff to manage the increase in presentations during an influenza pandemic. The current pandemic plans for the study sites do not have any specific provision for extra staffing for the EDs, which is concerning as the statistical modelling of a bioterrorism event of infectious disease showed that for an ED approximately half the size of study site one, there would need to be an increase of five nurses and two doctors per shift to manage for the first 72 hours of the event (Patvivatsiri, 2006). The use of statistical modelling using the study sites and the $\mathrm{MOH}^{\prime}$ s estimated national case rate of two million could provide guidance on how many additional staff are required across these sites during a pandemic (MOH, 2017).

\subsubsection{DHB level strategies}

The nurses held strong views that for their workplaces to cope during a pandemic, both their EDs and the hospitals themselves needed to be bigger, since these nurses feel they are already not fit for purpose during the current non-pandemic period. Overcrowding in EDs and a lack of inpatient bed availability are common problems in the health system today, so plans to significantly increase capacity during an influenza pandemic are crucial, as existing hospital infrastructure is likely to be insufficient (Ardagh \& Drew, 2015; Dewar, Barr \& Robinson, 2014). Current strategies for managing hospital capacity are focussed on decreased demand for hospital services by investing in increased primary healthcare engagement, however this will be irrelevant during a pandemic as demand will increase significantly during this time (Ardagh \& Drew, 2015). Australian-based research during the H1N1 influenza pandemic found that admitted patients boarding in the ED negatively impacted ED waiting times more than the increase in lower-acuity patients presenting for treatment. The authors felt that this "casts doubt on the effectiveness of policies to 'divert' primary care-suitable patients away from EDs as a way of avoiding crowding, especially during demand shocks" (Sivey, McAllister, Vally, Burgess \& Kelly, 2019, p. 8). This is significant, as pandemic planning at government level is heavily reliant on the diversion of lower acuity influenza patients away from EDs as a way for the NZ health system to cope during a pandemic $(\mathrm{MOH}, 2017)$. Due to financial constraints placed 
upon the health system, increasing the capacity of both study site EDs and wider hospitals is unlikely.

Given the likelihood that bigger hospitals cannot be provided, ED nurses wanted consideration to be given to methods to temporarily and rapidly create more space within or around ED for patients requiring care. This includes the use of non-clinical space within the existing hospital and the diversion of stable influenza patients away from ED. During the H1N1 influenza pandemic, many EDs in the United States reported structural space limitations affected their ability to care for the increase in patients. A Texas ED utilised a mobile response team housed in a field tent in the ED parking lot to carry out rapid influenza testing and treat low-acuity patients with influenza-like illness (ILI). This model saw $18 \%$ of the total ED patient volume, significantly alleviating pressure on the ED and reducing the risk of influenza transmission to other patients (Cruz et al., 2010). The concept of diversion of influenza patients away from ED has also been advocated for in the literature (Ranse et al., 2010). By establishing an alternative area for the care of influenza patients, hospitals aim to limit the spread of the disease to non-influenza patients in the ED. An alternative area would give the additional benefit that the lack of isolation rooms will no longer be a barrier to admission, as all patients in these areas will be positive for influenza and able to be cohorted together (Surgerman et al., 2011; Uyeki et al., 2019).

The use of incentives for staff to work during an influenza pandemic were considered by the nurses and have been researched previously. Australian researchers used quantitative surveying to investigate incentives and found that offering alternative accommodation for staff so that the risk of transmission to their families was decreased was an important incentive for ED staff to consider continuing to work (Martinese, Keijzers, Grant \& Lind, 2009). Other incentives that have been studied include financial compensation such as overtime pay, and engaging hospital social workers to assist with the emotional needs of frontline staff (Al-Shareef et al., 2017). Financial compensation was not at the forefront of the minds of the nurses in this study, rather the nurses were more concerned about the possible risk of infecting their families, so the use of alternative accommodation could be helpful in encouraging 
more staff to attend work during an influenza pandemic in NZ. However, the major incentive the nurses wanted was increased sick and domestic leave entitlements and having childcare on site. Increased leave entitlements could contribute to less presenteeism of sick nursing staff, potentially decreasing the risk that they will spread influenza to their colleagues and other patients. Increasing domestic leave entitlements and providing childcare on site would contribute to reducing the barriers some ED nurses find prevent them from working, and addressing these factors would show that the hospital system respects that ED nurses have roles and responsibilities as caregivers outside of their workplace. These considerations have not been investigated in the context of influenza pandemic planning and hence, this is a significant finding that brings a new perspective to the literature and should be considered for inclusion in future pandemic planning.

\subsubsection{Government level strategies}

The introduction of mandatory vaccination of healthcare workers was a strategy proposed by some senior ED nurses. Influenza is recognised as a vaccine-preventable illness, and yearly vaccination against the disease is encouraged by the $\mathrm{MOH}$ to prevent infections and reduce the burden of the disease (Charania \& Turner, 2018). Healthcare workers are offered free annual influenza vaccinations by their DHBs, but the average vaccination rate is only $66 \%$, well short of the $80 \%$ target set by the government. However, this rate is higher than the rest of NZ's population, of whom only one-quarter are vaccinated against seasonal influenza (Nowlan, Turner, Kiedrzynski \& Jennings, 2015). In addition to enhancing the health of frontline staff, many professional bodies representing infectious disease specialists, epidemiologists,

general medical physicians and paediatricians have expressed support for the mandatory vaccination of healthcare workers to decrease the risk of hospital-acquired influenza infections, which are associated with longer inpatient admissions, increased staff sickness and patient deaths (Ottenberg et al, 2011).

Not only does vaccination reduce the rates of influenza transmission from nurses to patients, but also from nurses to their colleagues. This is especially critical for the 
protection of vulnerable members of staff, including pregnant women and immunosuppressed nurses (Sullivan, Jacobson \& Poland 2009). Although a vaccine against a novel influenza virus will not be available immediately, with nurses expressing their concern in this research that influenza will be easily spread within the ED during a pandemic, mandatory vaccination could eventually help to prevent some of this transmission and reduce the overall disease burden. The introduction of mandatory vaccinations for children in Australia and other countries has caused controversy, with some arguing that this obstructs individual liberties and respect for autonomy, the freedom to make decisions about ones' own healthcare needs (Ward, Attwell, Meyer, Rokkas \& Leask, 2017). Conversely, it could be argued that based on the principles of both beneficence and non-maleficence, nurses have a responsibility to both their patients and their colleagues to comply with a scientifically-proven effective measure against the spread of influenza, unless vaccination is contraindicated on medical grounds. This is especially relevant during a pandemic where the virus will be more virulent and dangerous than seasonal influenza. Additionally, if all healthcare workers are legally mandated to be vaccinated once it becomes available, this reduces the possibility of healthcare workers using their unvaccinated status as a reason not to care for influenza patients within the hospital system. This is significant as there is no consideration of non-vaccinated staff in the study site pandemic plans, with possible exemption in place only for staff who have pre-existing health conditions which make them more vulnerable to a severe influenza infection (2018).

Another finding of this study is that ED nurses wanted free influenza vaccinations extended to their immediate family members. Currently, influenza vaccinations are free for healthcare workers through their DHB, and free for pregnant women, people aged 65 years and older, children aged under four years with a history of respiratory illness, and people aged under 65 years with significant co-morbidities (The Immunisation Advisory Centre, 2019). The nurses felt that the increased risk of exposing their family members to influenza warranted their inclusion in the vaccination schedule. The inclusion of immediate family members of frontline 
healthcare workers for free or subsidised vaccination has not been researched within the literature and requires investigation of the cost-benefit analysis.

Legislative change may also be required to meet another strategy ED nurses called for, the inclusion of a 'safety-net' provision to protect them from Health and Disability Commissioner (HDC) litigation for triaging away lower-acuity patients during an influenza pandemic. The nurses considered this reasonable since triaging away is outside their normal practice and has been mandated by their workplace and the $\mathrm{MOH}$. There is a precedent in previous HDC cases of nurses who did not recognise the severity of a patient's condition being censured for breaching the Code of Consumer's Rights by not upholding a patient's right to have health services provided at an appropriate standard and with reasonable care and skill (HDC, 2014, 2016, 2019). There are also cases in the literature of nurses being censured for decisions made during a disaster. After Hurricane Katrina, there were many deaths in hospitals of patients with acute presentations and chronic illnesses. A doctor and two nurses were charged with homicide due to "society's tendency to attempt to place blame for these tragedies", but were eventually exonerated after the difficulty of making decisions about whom to prioritise when resources are limited during a mass casualty incident was acknowledged (Holt, 2008, p. 182). Within the NZ context, the Crimes Act 1961 states that health professionals have a legal duty to provide the necessaries of life to the patients under their care, and if a patient's life or health is endangered or permanently impaired by a failure to provide the necessities of life, that health professional could, although unlikely, be charged with culpable homicide (Medical Council of New Zealand, 2006). Consideration of an amendment to take into account mass casualty events where resources are limited would help ED nurses, and by extension other health professionals, feel more comfortable with the difficulty of prioritising care in these situations.

Previous outbreaks of infectious disease, such as SARS, have resulted in widespread fear and panic within the community, especially when associated with high mortality rates. This can lead to discrimination of at-risk communities and stigmatization of infected persons, potentially contributing to delays in them seeking treatment and 
increased transmission of disease (Person et al., 2004). Lessons were learned from the SARS experience, and during the H1N1 influenza pandemic health officials were cognisant that reliable and frequent information updates and the formation of a relationship with the media were important in helping to prevent these same fears (Itzworth, Moa \& Maclntyre, 2018). My findings show that ED nurses felt that the government had a role in preventing community panic and scare-mongering by ensuring the spread of accurate pandemic information and practical healthcare advice, so that people feel comfortable caring for sick friends and relatives within their own homes and only presenting to ED if their condition requires it. The $\mathrm{MOH}$ acknowledges the importance of managing public health information, but ED nurses want the provision of accurate self-care advice across multiple languages to ensure all New Zealanders can understand the information. They feel this is crucial in limiting surges of lower-acuity influenza patients and the worried-well at EDs, allowing ED nurses to focus on those most in need of emergency care.

\subsection{Study strengths and limitations}

The choice of a qualitative descriptive design has allowed the collection of rich descriptions from ED nurses to provide a comprehensive summary of their perspectives on an influenza pandemic, a topic that has not previously been well explored within the ED setting or in NZ. The research findings provide a first-hand account of the problems faced by ED nurses in everyday practice, the potential future problems that could occur during an influenza pandemic, and recommendations for practice that come directly from staff working at the frontline of the healthcare system. This addresses the significant lack of NZ ED nurses' perspectives within the literature.

There is ongoing debate amongst qualitative researchers about what represents an appropriate sample size to lend adequate rigour to a study. Guidance was obtained from research with similar methodogies in the literature, with the sample sizes for qualitative studies using semi-structured interviews ranging from 10 to 46 participants. With a total sample of approximately 80 triage-trained nurses across both study sites, the decision to sample approximately one in five was considered appropriate. This 
sample size of 16 nurses allowed the complex topic of the impact of an influenza pandemic on NZ EDs to be explored in depth using open-ended questions, whilst allowing for revision of the questions as new information emerged during interviews. The sample size was sufficient in that there was variability across gender and years of experience within ED and triage, enhancing the transferability and potential generalisability of the findings to other EDs in NZ.

The use of a convenience sample was necesssary due to geographic and financial constraints, as well as the choice of face-to-face, semi-structured interviews for data collection. Data collection was limited to two urban study sites located in the lower North Island, and this impacts upon the generalisability of the research, as other areas of NZ have different population demographics that could influence responses. All 16 participants identified as NZ, Australian or British European, so this research is limited in that there is no representation from Māori, Pacific or Asian ethnic groups, who represent an important sector of the NZ society. Inclusion of nurses from these ethnicities may have altered the findings through different cultural persepctives.

As participation in this research was on a voluntary basis, there is a risk for selfselection bias, where nurses who hold a particular interest in the research topic are more likely to participate in the study. Voluntary participation was a criteria of gaining ethics approval, so self-selection was unavoidable and it is unknown if this influenced the results. Given the various perspectives shared on many topics, if there was bias this was most likely minimal.

Demonstrating research rigour can be challenging in qualitative studies, and this is considered a limitation of qualitative methodologies (Polit \& Beck, 2004). To overcome this, a transparent process was taken with data analysis and reporting. Data was analysed first by reading the transcripts and documenting first impressions, before the transcripts were carefully coded on qualitative software. All stages of coding and theme development were reviewed by the supervisor to ensure their understanding was in congruence with the researcher. Journaling of all coding and thematic decisions was used to provide an audit trail and confirm dependability. 


\subsection{Recommendations}

The strategies the ED nurses consider as necessary for the successful management of an influenza pandemic are reasonable and achievable goals that EDs, DHBs and the $\mathrm{MOH}$ should invest in as soon as possible to ensure the health system is ready if a pandemic were to occur in future. In addition to these strategies, further recommendations are included based upon the research findings.

\subsubsection{For EDs and everyday ED nursing practice}

1. EDs need to urgently develop staff education and training packages specifically to meet infection control standards and embed safe habits within ED staff. These must be ED-specific rather than generic hospital-wide policies that includes information on initiating infection control precautions at triage and maintaining precautions in challenging clinical areas, such as the resuscitation rooms.

2. If a larger ED cannot be provided, EDs must urgently develop methods to easily separate infectious and non-infectious patients:

a. The use of transparent plastic wall dividers to segregate ED waiting rooms would allow separate infectious and non-infectious areas, whilst allowing for triage nurses to continue to monitor all waiting room patients in the event of patient deterioration

b. The triage and reception areas must be either placed behind windows or further back across existing desks to allow for staff to be seated further from where new patients arrive. These methods would still allow for the exchange of patient details and presenting complaints, whilst decreasing the likelihood of transmission of droplet-borne illnesses within the high-risk triage area

c. EDs must develop protocols for infectious patient placement, with preferential use of certain cubicles that have easy access to PPE. These protocols should also indicate areas for vulnerable or non-infectious patient placement that reduce the liklihood of disease transmission 


\subsubsection{For pandemic planning}

1. All DHBs in NZ need to urgently review their pandemic plans to take into consideration:

a. The development of relief rosters of extra staff, or redeployment of nursing staff from other services to assist to fill gaps within the ED nursing workforce during a pandemic. With the $\mathrm{MOH}$ plan to cancel elective surgeries during a pandemic, perioperative and postoperative nurses could be used for this purpose.

b. The diversion of stable influenza patients who require hospital care away from ED. The use of a field tent or diverting to a seperate influenza-only ward would assist in reducing the ED workflow and help to reduce the transmission of influenza to other vulnerable patients.

c. DHBs must also build relationships with private hospital facilities in their regions, as these facilities may be able to take transfers of influenza patients upon the cancellation of elective surgical services

2. The MOH needs to urgently develop comprehensive pandemic guidelines that ensures the safety of staff and patients. This needs to include specific details about triaging systems and guidelines, pandemic training for community healthcare staff and the prioritisation of finite health resources, including staff, admission beds and vital equipment.

3. ED nurses and their professional body, the College of Emergency Nurses New Zealand (CENNZ), must be involved in DHB and government-level pandemic planning. By involving ED nurses in the planning stages, their views, concerns and ideas can be considered and incorporated in future plans, allowing these nurses to be engaged and more likely to feel safe to attend work and assist the public during a future influenza pandemic. 


\subsubsection{For future research}

The findings of this research identified the frequent use of behaviours used to cope with high workloads, termed workarounds within other nursing specialties. With little research available on workarounds in the ED environment, this phenomenon requires further research to determine the frequency and types of workarounds employed by ED nurses, and solutions to reduce their occurrence and promote patient safety daily, as well as during an influenza pandemic.

Similarly, little research has been undertaken on the presenteeism of ED nurses. A qualitative descriptive study into this phenomenon could undercover additional EDspecific contributing factors that could then be addressed by DHBs to ensure a more productive and healthier nursing workforce.

This research is limited to 16 ED nurses across two study sites. A Delphi study of ED nurses across NZ should be undertaken to determine their expert consensus on the strategies considered important by these nurses, and any further additional strategies that may be required but not considered by the nurses within this study.

\subsection{Conclusion}

This research contributes signficantly to expanding the current body of knowledge concerning how ED nurses feel about the threat of an influenza pandemic and what strategies they consider important to mitigate the risk to themselves, their patients and their families. The frequency of workarounds to manage seasonal influenza within EDs is a significant new finding to emerge from this research, as is the identification of various ED-specific ethical concerns that have not been considered or accounted for in existing influenza pandemic plans. Acceptance and actioning of the recommendations by the $\mathrm{MOH}$ and DHBs could help to shape future pandemic planning to maximise the safety of both ED nursing staff and patients.

Pandemic planning requires different considerations tailored to individual countries and their unique healthcare systems, and this research is the first that specifically 
highlights the perspectives of NZ ED nurses, whom the health system is heavily reliant upon to provide initial and ongoing care in mass casualty emergencies including pandemics.

DHBs and the $\mathrm{MOH}$ must read this research and discuss the process of adopting the recommendations into their pandemic plans in order to avoid the many practical and ethical issues likely to occur during a future pandemic. The recent emergence of the coronavirus is a timely reminder that this work must occur with urgency in order to ensure the NZ healthcare system is well prepared in the event this or another virus evolves into a worldwide health emergency. 


\section{REFERENCES}

Aacharya, R. P., Gastmans, C., \& Denier, Y. (2011). Emergency department triage: An ethical analysis. BMC Emergency Medicine, 11(1), 16. Retrieved from http://www.biomedcentral.com/1471-227X/11/16

Al-Shareef, A. S., Alsulimani, L. K., Bojan, H. M., Masri, T. M., Grimes, J. O., Molloy, M. S., \& Ciottone, G. R. (2017). Evaluation of hospitals' disaster preparedness plans in the holy city of Mekkah (Mecca): A cross-sectional observation study. Prehospital and Disaster Medicine, 32(1), 33-45. doi: $10.1017 / \mathrm{S} 1049023 \times 16001229$

Arbon, P., Ranse, J., Cusack, L., Considine, J., Shaban, R. Z., Woodman, R. J. . . . Mitchell, B. (2013). Exploring staff willingness to attend work in a disaster: A study of nurses employed in four Australian emergency departments. Australasian Emergency Nursing Journal, 16(3), 103-109. doi: 10.1016/j.aenj.2013.05.004

Ardagh, M., \& Drew, L. (2015). What have five years of the shorter stays in the emergency department health target done to us? New Zealand Medical Journal, 128(1421), 47-54. Retrieved from https://www.nzma.org.nz/journal/read-the-journal/all-issues/20102019/2015/vol-128-no-1421-4-september-2015/6640

Ardagh, M. W., Richardson, S. K., Robinson, V., Than, M., Gee, P., Henderson, S. . . Deely, J. M. (2012). The initial health-system response to the earthquake in Christchurch, New Zealand, in February, 2011. Lancet, 379(9831), 2109-2115. doi: 10.1016/S0140-6736(12)60313-4 
Aronsson, G., Gustafsson, K., \& Dallner, M. (2000). Sick but yet at work: An empirical study of sickness presenteeism. Journal of Epidemiology and Community Health, 54(7), 502-509. doi: 10.1136/jech.54.7.502

Asfaw, A., Rosa, R., \& Pana-Cryan, R. (2017). Potential economic benefits of paid sick leave in reducing absenteeism related to the spread of influenza-like illness. Journal of Occupational and Environmental Medicine, 59(9), 822-829. doi: 10.1097/JOM.0000000000001076

Asgari, S., Shafipour, V., Taraghi, Z., \& Yazdani-Charati, J. (2019). Relationship between moral distress and ethical climate with job satisfaction in nurses. Nursing Ethics, 26(2), 346-356. doi: 10.1177/0969733017712083

Asselin, M. (2003). Insider research: Issues to consider when doing qualitative research in your own setting. Journal for Nurses in Staff Development, 19(2), 99-103. Retrieved from https://journals.Iww.com/jnsdonline/Abstract/2003/03000/Insider_Research_ _Issues_to_Consider_When_Doing.8.aspx

Australasian College of Emergency Medicine. (2016). Guidelines on the implementation of the Australasian triage scale in emergency departments. Retrieved from https://acem.org.au/getmedia/51dc74f7-9ff0-42ce-872a0437f3db640a/G24_04_Guidelines_on_Implementation_of_ATS_Jul-16.aspx

Australasian College of Emergency Medicine. (2019). Position statement access block. Retrieved from https://acem.org.au/getmedia/c0bf8984-56f3-4b788849-442feaca8ca6/S127_v01_Statement_Access_Block_Mar_14.aspx

Australian Government Department of Health. (2019). Australian health management plan for pandemic influenza. Retrieved from https://www1.health.gov.au/internet/main/publishing.nsf/Content/ohpahmppi.htm 
Bell, J. J., \& Selvarangan, R. (2014). Evaluation of the alere i influenza a\&B nucleic acid amplification test by use of respiratory specimens collected in viral transport medium. Journal of Clinical Microbiology, 52(11), 3992-3995. doi: 10.1128/JCM.01639-14

Boehm, N., Cabral, M., Harkinson, M. \& Sakers, C. (2010). H1N1 2009: One pediatric emergency department's experience. Journal of Emergency Nursing, 36(2), 125-129. doi: 10.1016/j.jen.2009.07.016

Braun, V., \& Clarke, V. (2008). Using thematic analysis in psychology. Qualitative Research in Psychology, 3(2), p. 77-101. doi: 10.1191/1478088706qp063oa

Brink, P. J. (1998). Exploratory designs. In P. J. Brink, \& M. J. Wood (Eds.), Advanced design in nursing research (2nd ed., pp. 308-334). Thousand Oaks, CA: Sage Publications.

Brinkman, S. (2014). Unstructured and semi-structured interviewing. In P. Leavy (Ed.), The Oxford handbook of qualitative research, (pp. 277-299). Oxford, England: Oxford University Press.

Burström, L., Starrin, B., Engström, M. L., \& Thulesius, H. (2013). Waiting management at the emergency department: A grounded theory study. BMC Health Services Research, 13 (1), 95. doi: 10.1186/1472-6963-13-95

Centers for Disease Control and Prevention. (2019). 2009 H1N1 pandemic (H1N1pdm09 virus). Retrieved from https://www.cdc.gov/flu/pandemicresources/2009-h1n1-pandemic.html

Charania, N. A., \& Turner, N. M. (2018). 100 years since the 1918 influenza pandemic - progress made, yet questions remain. A synopsis of the $4^{\text {th }}$ New Zealand influenza symposium, February 2018. New Zealand Medical Journal, 131(1481), 
50-55. Retrieved from https://www.nzma.org.nz/journal/read-the-journal/allissues/2010-2019/2018/vol-131-no-1481-31-august-2018/7680

Chen, Y. C., Huang, L. M., Chan, C. C., Su, C. P., Chang, S. C., Chang, Y. Y. . . L Lee, Y. T. (2004). SARS in hospital emergency room. Emerging infectious diseases, 10(5), 782-788. doi: 10.3201/eid1005.030579.

Christian, M. D., Hawryluck, L., Wax, R.S., Cook, T., Lazar, N. M., Herridge, M. S. . . Burkle, F. M. (2006). Development of a triage protocol for critical care during an influenza pandemic. Canadian Medical Association Journal, 175(11), 13771381. doi: $10.1503 / \mathrm{cmaj} .060911$

Clarke, V., \& Braun, V. (2013). Successful qualitative research: a practical guide for beginners. London, England: SAGE Publications.

Coburn, B. J., Wagner, B. G., \& Blower, S. (2009). Modeling influenza epidemics and pandemics: insights into the future of swine flu (H1N1). BMC Medicine, 7, 30. doi: $10.1186 / 1741-7015-7-30$

College of Emergency Nurses New Zealand. (2009). CENNZ-NZNO position statement: Triaging away. Retrieved from https://www.nzno.org.nz/Portals/0/Files/Documents/Groups/Emergency\%20N urses/Publications/3_\%202009\%20CENNZ\%20Position\%20statement\%20on\%2 Otriaging\%20away.pdf

Considine, J., Shaban, R. Z., Patrick, J., Holzhauser, K. Aitken, P., Clark, M. . . . FitzGerald, G. (2011). Pandemic (H1N1) 2009 influenza in Australia: Absenteeism and redeployment of emergency medicine and nursing staff. Emergency Medicine Australasia, 23(5), 615-623. doi: 10.1111/j.17426723.2011.01461.x 
Corley, A., Hammond, N. E., \& Fraser, J. F. (2010). The experiences of health care workers employed in an Australian intensive care unit during the H1N1 influenza pandemic of 2009: A phenomenological study. International Journal of Nursing Studies, 47(5), 577-585. doi: 10.1016/j.ijnurstu.2009.11.015

Coup, A. \& Schneider, Z. (2007). Ethical and legal issues in research. In Z. Schneider, D. Whitehead, D. Elliott, G. LoBiondo-Wood, \& J. Haber (Eds.), Nursing and midwifery research: Methods and appraisal for evidence based practice (3rd ed., pp. 80-101). Sydney, Australia: Elsevier.

Crimes Act, No. 43. (1961). Retrieved from http://www.legislation.govt.nz/act/public/1961/0043/latest/DLM327382.html

Croskerry, P. (2002). Achieving quality in clinical decision making: Cognitive strategies and detection of bias. Academic Emergency Medicine, 9(11), 1184-1204. doi: 10.1197/aemj.9.11.1184

Cruz, A. T., Patel, B., DiStefano, M. C., Codispoti, C. R., Shook, J. E., DemmlerHarrison, G.J., \& Sirbaugh, P. E. (2010). Outside the box and into thick air: Implementation of an exterior mobile paediatric emergency response team for North American H1N1 (Swine) influenza virus in Houston, Texas. Annals of Emergency Medicine, 55(1), 23-31. doi: 10.1016/j.annemergmed.2009.08.003

Cypress, B. (2017). Rigor or reliability and validity in qualitative research: Perspectives, strategies, reconceptualization, and recommendations. Dimensions of Critical Care Nursing, 36(4), 253-263. doi: 10.1097/DCC.0000000000000253

Debono, D. S., Greenfield, D., Travaglia, J. F., Long, J. C., Black, D., Johnson, J., \& Braithwaite, J. (2013). Nurses' workarounds in acute healthcare settings: A scoping review. BMC Health Services Research, 13(1), 175. doi: 10.1186/14726963-13-175 
Dewar, B., Barr, I., \& Robinson, P. (2014). Hospital capacity and management preparedness for pandemic influenza in Victoria. Australian and New Zealand Journal of Public Health, 38(2), 184-190. doi: 10.1111/1753-6405.12170

Dicker, R. C., Coronado, F., Koo, D., \& Gibson Parrish, R. (2012). Principles of epidemiology in public health practice: an introduction to applied epidemiology and biostatistics ( $3^{\text {rd }}$ ed.). Atlanta, GA: CDC.

District Health Board. (2018). HHS influenza surge response plan. Retrieved from dhb.sharepoint.com/sites/ intranet/Pages/Home.aspx

Elliott, D. (2007). Reviewing the literature. In Z. Schneider, D. Whitehead, D. Elliott, G. LoBiondo-Wood, \& J. Haber (Eds.), Nursing and midwifery research: Methods and appraisal for evidence based practice (3rd ed., pp. 46-61). Sydney, Australia: Elsevier.

Elliott, D., \& Thompson, D. (2007). Common quantitative methods. In Z. Schneider, D. Whitehead, D. Elliott, G. LoBiondo-Wood, \& J. Haber (Eds.), Nursing and midwifery research: Methods and appraisal for evidence based practice (3rd ed., pp. 156-174). Sydney, Australia: Elsevier.

Elliott, R., \& Timulak, L. (2005). Descriptive and interpretive approaches to qualitative research. In J. Miles \& P. Gilbert (Eds.), A Handbook of research methods in clinical and health psychology (pp. 157-160). Oxford, England: Oxford University Press.

Elo, S., \& Kyngäs, H. (2008). The qualitative content analysis process. Journal of Advanced Nursing, 62(1), 107-115. doi: 10.1111/j.1365-2648.2007.04569.x

Erasmus, V., Daha, T.J., Brug, H., Richarus, J. H., Behrendt, M. D., Vos, M. C., \& van Beeck, E. F. (2010). Systematic review of studies on compliance with hand 
hygiene guidelines in hospital care. Infection Control and Hospital Epidemiology, 31(3), 283-294. doi: 10.1086/650451

Fagbuyi, D. B., Brown, K. M., Mathison, D. J., Kingsnorth, J., Morrison, S., Saidinejad, M. . . Chamberlain, J. M. (2011). A rapid medical screening process improves emergency department patient flow during surge associated with novel H1N1 influenza virus. Annals of Emergency Medicine, 57(1), 52-59. doi: 10.1016/j.annemergmed.2010.08.026

Filice, C. E., Vaca, F. E., Curry, L., Platis, S., Lurie, N., \& Bogucki, S. (2013). Pandemic planning and response in academic pediatric emergency departments during the 2009 H1N1 influenza pandemic. Academic Emergency Medicine, 20(1), 5462. doi: 10.1111/acem.12061

Forero, R., McCarthy, S., \& Hillman, K. (2011). Access block and emergency department overcrowding. Critical Care, 15(2), 2-6. doi: 10.1186/cc9998

Foss, C., \& Krogstad, U. (2000). The invisible patient - patients' experiences of staying in a hallway bed. Europe PMC, 120(22), 2639-2641. Retrieved from https://europepmc.org/article/med/11077508

Fusco, F. M., \& Puro, V. (2012). Infection control in the emergency department. Canadian Medical Association Journal, 184(9), 1065. doi: 10.1503/cmaj.1122038

Godkin, D., \& Markwell, H. (2003). The duty to care of health professionals: Ethical issues and guidelines for policy development. Toronto: University of Toronto.

Goodacre, S., Irving, A., Wilson, R., Beever, D., \& Challen, K. (2015). The pandemic influenza triage in the emergency department (PAINTED) pilot cohort study. Health Technology Assessment, 19(3), 1-96. doi: 10.3310/hta19030 
Greenacre, M., \& Fleshner, K. (2017). Distributive justice in disaster triage:

Utilitarianism competes with egalitarianism, autonomy, and the physicianpatient relationship. University of Western Ontario Medical Journal, 86(1), 35-

37. Retrieved from http://www.uwomj.com/wpcontent/uploads/2017/08/v86no1_13.pdf

Halbesleben, J. R. (2010). The role of exhaustion and workarounds in predicting occupational injuries: A cross-lagged panel study of health care professionals. Journal of Occupational Health Psychology, 15(1), 1-16. doi: 10.1037/a0017634

Health and Disability Commissioner. (2014). 12HDC01172 Triage assessment of patient with reported exposure to meningitis: Delays in treatment. Retrieved from https://www.hdc.org.nz/media/1708/12hdc01172.pdf

Health and Disability Commissioner. (2016). 14HDC01187 Assessment and treatment of a young child with fever and respiratory symptoms. Retrieved from https://www.hdc.org.nz/media/1562/14hdc01187.pdf

Health and Disability Commissioner. (2019). 17HDC00067 Assessment of woman presenting with shortness of breath and cough. Retrieved from https://www.hdc.org.nz/media/5203/17hdc00067.pdf

Hobbs, M., Ahuriri-Driscoll, A., Marek, L., Campbell, M., Tomintz, M., \& Kingham, S. (2019). Reducing health inequity for Māori people in New Zealand. The Lancet, 394(10209), 1613-1614. doi: 10.1016/S0140-6736(19)30044-3

Holt, G. R. (2008). Making difficult ethical decisions in patient care during natural disasters and other mass casualty events. Otolaryngology - Head and Neck Surgery, 139(2), 181-186. doi: 10.1016/j.otohns.2008.04.027 
Hsieh, H. F., \& Shannon, S. E. (2005). Three approaches to qualitative content analysis. Qualitative Health Research, 15(9), 1277-88. doi: $10.177 / 1049732305276687$

Hudson, M. L., \& Russell, K. (2009). The Treaty of Waitangi and research ethics in Aotearoa. Journal of Bioethical Inquiry, 6(1), 61-68. doi: 10.1007/s11673-0089127-0

Itzworth, R., Moa, A., \& Maclntyre, C. R. (2018). Australia's influenza pandemic preparedness plans: An analysis. Journal of Public Health Policy, 39(1), 111-124. doi: 10.1057/s41271-017-0109-5

Joffe, H., \& Yardley, L. (2004). Content and thematic analysis. In D. F. Marks \& L. Yardley (Eds.), Research methods for clinical and health psychology (pp. 56-68). London, England: SAGE Publications

Jootun, D., McGhee, G., \& Marland, G. R. (2009). Reflexivity: Promoting rigour in qualitative research. Nursing Standard, 23(23), 42-46. doi:

10.7748/ns2009.02.23.23.42.c6800

Kahol, K., Vankipuram, M., Patel, V. L., \& Smith, M. L. (2011). Deviations from protocol in a complex trauma environment: Errors or innovations? Journal of Biomedical Informatics, 44(3), 425-431. doi: 10.1016/j.jbi.2011.04.003

Koch, T. (2006). Establishing rigour in qualitative research: The decision trail. Journal of Advanced Nursing, 53(1), 91-103. doi: 10.1111/j.1365-2648.2006.03681

Lam, K. K., \& Hung, S. Y. M. (2013). Perceptions of emergency nurses during the human swine influenza outbreak: A qualitative study. International Emergency Nursing, 21(4), 240-246. doi: 10.1016/j.ienj.2012.08.008

Liamputtong, P. (2013). Qualitative research methods (4th ed.). Oxford, England: Oxford University Press. 
Liang, S. Y., Theodoro, D. L., Schuur, J. D. \& Marschall, J. (2014). Infection prevention in the emergency department. Annals of Emergency Medicine, 64(3), 299-313. doi: 10.1016/j.annemergmed.2014.02.024

Lincoln, Y.S. (2009). Ethical practices in qualitative research. In D.M Mertens \& P.E. Ginsberg (Eds.), The handbook of social research ethics (pp. 150-169). Thousand Oaks, CA: SAGE Publications.

Lincoln, Y.S., \& Guba, E.G. (1985). Naturalistic Inquiry (1st ed.). Thousand Oaks, CA: Sage Publications.

Lowe, R. A., Bindman, A. B., Ulrich, S. K., Norman, G., Scaletta, T. A., Keane, D., Washing, D., \& Grumbach, K. (1994). Refusing care to emergency department patients: Evaluation of published triage guidelines. Annals of Emergency Medicine, 23(2), 286-293. doi: 10.1016/S0196-0644(94)70042-7

Madhav, N., Oppenheim, B., Gallivan, M., Mulembakani, P., Rubin, E., \& Wolfe, N. (2017). Pandemics: Risks, impacts, and mitigation. In Jamison, D.T., Gelband, Horton, S., Jha, P., Laxminarayan, R., Mock, C.N., and Nugent, R (Eds.), Disease control priorities: Improving health and reducing poverty (3rd ed., pp 315-345). Washington, D.C.: World Bank Group.

Marshall, C., Kelso, A., McBryde, E., Barr, I. G., Eisen, D. P., Sasadeusz, J. . . Richards, M. (2011). Pandemic (H1N1) 2009 risk for frontline health care workers. Emerging Infectious Diseases, 17(6), 1000-1006. doi: 10.3201/eid1706.101030

Martel, J., Bui-Xuan, E. F., Carreau, A. M., Carrier, J. D., Larkin, E., Vlachos-Mayer, H., \& Dumas, M. E. (2013). Respiratory hygiene in emergency departments: Compliance, beliefs, and perceptions. American Journal of Infection Control, 41(1), 14-18. doi: 10.1016/j.ajic.2011.12.019 
Martinese, F., Keijzers, G., Grant, S., \& Lind, J. (2009). How would Australian hospital staff react to an avian influenza admission, or an influenza pandemic? Emergency Medicine Australasia, 21(1), 12-24. doi: 10.1111/j.1742$6723.2008 .01143 . x$

May, L., Lung, D., \& Harter, K. (2012). An intervention to improve compliance with transmission precautions for influenza in the emergency department: Successes and challenges. Journal of Emergency Medicine, 42(1), 79-85. doi: 10.1016/j.jemermed.2010.02.034

McCullough, L. B. (2010). Taking seriously the "what then?" question: An ethical framework for the responsible management of medical disasters. Journal of Clinical Ethics, 21(4), 321-327). Retrieved from https://www.ncbi.nlm.nih.gov/pubmed/21313866

McMullan, C., Brown, G. D., \& O’Sullivan, D. (2016). Preparing to respond: Irish nurses' perceptions of preparedness for an influenza pandemic. International Emergency Nursing, 26(1), 3-7. doi: 10.1016/j.ienj.2015.10.004

Medical Council of New Zealand. (2006). A doctor's duty to help in a medical emergency. Retrieved from https://www.mcnz.org.nz/assets/standards/afd1af693d/A-doctors-duty-tohelp-in-a-medical-emergency.pdf

Mentis, H. M., Reddy, M., \& Rosson, M. B. (2010). Invisible emotion: Information and interaction in an emergency room. In Inkpen, K., Gutwin, C., \& Tang, J. (Eds.), CSCW'10: The 2010 ACM conference on computer supported cooperative work (pp. 311-320). New York, NY: Association for Computing Machinery. doi: $10.1145 / 1718918 / 1718917$

Merckx, J., Wali, R., Schiller, I., Caya, C., Gore, G. C., Chartrand, C. . . Papenburg, J. (2017). Diagnostic accuracy of novel and traditional rapid tests for influenza 
infection compared with reverse transcriptase polymerase chain reaction: A systematic review and meta-analysis. Annals of Internal Medicine, 167(6), 394409. doi: $10.7326 / \mathrm{M} 17-0848$

Ministry of Health. (2008). National health emergency plan: Guidance on communitybased assessment centres and other support services. Retrieved from https://www.health.govt.nz/system/files/documents/publications/nhepcommunity-based-assessment-dec08.pdf

Ministry of Health. (2013). Public hospitals. Retrieved from https://www.health.govt.nz/our-work/hospitals-and-specialist-care/publichospitals

Ministry of Health. (2015). Emergency department services: Specialist medical and surgical services tier level two. Retrieved from https://nsfl.health.govt.nz/service-specifications/current-servicespecifications/specialist-medical-services-specifications

Ministry of Health. (2017). New Zealand influenza pandemic plan: A framework for action. Retrieved from https://www.health.govt.nz/system/files/documents/publications/influenzapandemic-plan-framework-action-2nd-edn-aug17.pdf

Ministry of Health. (2020). Novel coronavirus (2019-nCoV). Retrieved from https://www.health.govt.nz/our-work/diseases-and-conditions/novelcoronavirus-2019-ncov

Morley, C., Stankovich, J., Peterson, G., \& Kinsman, L. (2018). Planning for the future: Emergency department presentation patterns in Tasmania, Australia. International Emergency Nursing, 38(1), 34-40. doi: 10.1016/j.ienj.2017.09.001 
Neergaard, M. A., Olesen, F., Andersen, R. S., \& Sondergaard, J. (2009). Qualitative description-the poor cousin of health research? BMC Medical Research Methodology, 9(1), 52. doi: 10.1186/1471-2288-9-52

New Zealand Nurses Organisation. (2016) Guideline: Obligations in a pandemic or disaster, 2016. Retrieved from https://www.nzno.org.nz/LinkClick.aspx?fileticket=3YrTAd7YRLI\%3D\&portalid= 0

New Zealand Nurses Organisation. (2018). District Health Boards/NZNO nursing and midwifery multi-employer collective agreement. Retrieved from https://www.nzno.org.nz/groups/health_sectors/dhb

Nowell, L. S., Norris, J. M., White, D. E., \& Moules, N. J. (2017). Thematic analysis: striving to meet the trustworthiness criteria. International Journal of Qualitative Methods, 16(1), 1-13. doi: 10.1177/1609406917733847

Nowlan, M., Turner, N., Kiedrzynski, T., \& Jennings, L. (2015). Synopsis of New Zealand's inaugural influenza symposium: Influenza is a severe vaccinepreventable disease. The New Zealand Medical Journal, 128(1410), 30-39. Retrieved from https://www.nzma.org.nz/journal/read-the-journal/allissues/2010-2019/2015/vol-128-no-1409-20-feb-2015/6457

Núñez, S., Moreno, A., Green, K., \& Villar, J. (2000). The stethoscope in the emergency department: A vector of infection? Epidemiology \& Infection, 124(2), 233-237. doi: 10.1017/s0950268800003563

Nursing Council of New Zealand. (2011). Guidelines for cultural safety, the Treaty of Waitangi, and Maori health in nursing education and practice. Retrieved from http://www.nursingcouncil.org.nz/Publications/Standards-and-guidelines-fornurses 
O’Boyle, C., Robertson, C., \& Secor-Turner, M. (2006). Public health emergencies: Nurses' recommendations for effective actions. American Association of Occupational Health Nurses, 54(8), 347-353. Retrieved from http://aaohn.org/page/wh-and-s-journal

Osterholm, M. T. (2005). Preparing for the next pandemic. The New England Journal of Medicine, 352(18), 1839-1842. doi: 10.1056/NEJMp058068

Ottenberg, A. L., Wu, J. T., Poland, G. A., Jacobson, R. M., Koenig, B. A., \& Tilburt, J. C. (2011). Vaccinating health care workers against influenza: The ethical and legal rationale for a mandate. American Journal of Public Health, 101(2), 212-216. doi: 10.2105/AJPH.2009.190751

Paparella, S. F. (2018). First- and second-order problem solving: When rework and workarounds become an opportunity for improving safety. Journal of Emergency Nursing, 44(6), 652-654. doi: 10.1016/j.jen.2018.07.008

Patvivatsiri, L. (2006). A simulation model for bioterrorism preparedness in an emergency room. In Lawson, B., Liu, J., Perrone, F., \& Wieland, F. (Eds.), 2006 Winter simulation conference (pp. 501-508). New York, NY: Institute of Electrical and Electronics Engineers. doi: 10.1109/WSC.2006.323122

Perry, L., Lamont, S., Brunero, S., Gallagher, R., \& Duffield, C. (2015). The mental health of nurses in acute teaching hospital settings: A cross-sectional survey. BMC Nursing, 14(1), 1-8. doi: 10.1186/s12912-015-0068-8

Person, B., Sy, F., Holton, K., Govert, B., Liang, A., Garza, B. . . Zauderer, L. (2004). Fear and stigma: The epidemic within the SARS outbreak. Emerging Infectious Diseases, 10(2), 358-363. doi: 10.3201/eid1002.030750

Picheansathian, W. (1995). Compliance with universal precautions by emergency room nurses at Maharaj Nakorn Chiang Mai Hospital. Journal of the Medical 
Association of Thailand, 78(Suppl. 2), 118-122. Retrieved from https://www.ncbi.nlm.nih.gov/pubmed/7561585

Polit, D. F., \& Beck, C. T. (2004). Nursing research: Principles and methods (7th ed.). Philadelphia, PA: Lippincott Williams \& Wilkins.

Prior, L. (2014). Content analysis. In P Leavy (Ed.), The Oxford handbook of qualitative research (pp. 359-379). Oxford, England: Oxford University Press.

Quick, J., \& Hall, S. (2015). Part three: The quantitative approach. Journal of Perioperative Practice, 25(10), 192-196. doi: 10.1177/175045891502501002

Rainbow, J. G., \& Steege, L. M. (2017). Presenteeism in nursing: An evolutionary concept analysis. Nursing Outlook, 65(5), 615-623. doi:

10.1016/j.outlook.2017.03.005

Ranse, J., Lenson, S., Luther, M., \& Xiao, L. (2010). H1N1 2009 influenza (human swine influenza): A descriptive study of the response of an influenza assessment clinic collaborating with an emergency department in Australia. Australasian Emergency Nursing, 13(3), 46-52. doi: 10.1016/j.aenj.2010.03.001

Rantanen, I., \& Tuominen, R. (2011). Relative magnitude of presenteeism and absenteeism and work-related factors affecting them among health care professionals. International Archives of Occupational and Environmental Health, 84(1), 225-230. doi: 10.1007/s00420-010-0604-5

Rees, G. H. (2014). Organisational readiness and lean thinking implementation: Findings from three emergency department case studies in New Zealand. Health Services Management Research, 27(1-2), 1-9. doi:

$10.1177 / 0951484814532624$ 
Richards, J. R., van der Linden, M. C., \& Derlet, R. W. (2014). Providing care in emergency department hallways: Demands, dangers, and deaths. Advances in Emergency Medicine, 2014(1). doi: 10.1155/2014/495219

Rothman, R. E., Irvin, C. B., Moran, G. J., Sauer, L., Bradshaw, Y. S., Fry, R. B. . . Hirshon, J. M. (2006). Respiratory hygiene in the emergency department. Annals of Emergency Medicine, 48(5), 570-582. doi: 10.1016/j.annemergmed.2006.05.018

Rottman, S. J., Shoaf, K. I., Schlesinger, J., Klein Selski, E., Perman, J., Lamb, K., \& Cheng, J. (2010). Pandemic influenza triage in the clinical setting. Prehospital and Disaster Medicine, 25(2), 99-104. doi: 10.1017/S1049023X0002427

Ruderman, C., Tracy, C. S., Bensimon, C. M., Bernstein, M., Hawryluck, L., Shaul, R. Z., \& Upshur, R. E. G. (2006). On pandemics and the duty to care: Whose duty? who cares? BMC Medical Ethics, 7(1), 5. doi: 10.1186/1472-6939-7-5

Sandelowski, M. (2000). Focus on research methods: Whatever happened to qualitative description? Research in Nursing and Health, 23(4), 334-340. doi: $10.1002 / 1098-240 \times(200008) 23: 43.0$

Sandelowski, M. (2010). What's in a name? Qualitative description revisited. Research in Nursing and Health, 33(1), 77-84. doi: 10.1002/nurs.20362

Sandoval, C., Barrera, A., Ferrès, M., Cerda, J., Retamal, J., Garcia-Sastre, A. . . . Hirsch, T. (2016). Infection in health personnel with high and low levels of exposure in a hospital setting during the H1N1 influenza a pandemic. PLOS One, 11(1). doi: 10.1371/journal.pone.0147271

Seale, H., Leask, J., Po, K., \& Maclntyre, C. R. (2009). "Will they just pack up and leave?" - attitudes and intended behaviour of hospital health care workers 
during an influenza pandemic. BMC Health Services Research, 9(1). doi: $10.1186 / 1472-6963-9-30$

Shenton, A. (2004). Strategies for ensuring trustworthiness in qualitative research projects. Education for Information, 22(2), 63-75. doi: 10.3233/EFI-2004-22201

Sivey, P., McAllister, R., Vally, H., Burgess, A., \& Kelly, A.-M. (2019). Anatomy of a demand shock: Quantitative analysis of crowing in hospital emergency departments in Victoria, Australia during the 2009 influenza pandemic. PLOS ONE, 14(9), e0222851. doi: 10.1371/journal/pone.0222851

Sokol, D. K. (2006). Virulen epidemics and scope of healthcare workers' duty of care. Emerging Infectious Diseases, 12(8), 1238-1241. doi: 10/3201/eid1208.060360

Sugerman, D., Nadeau, K. H., Lafond, K., Cameron, W., Soetebier, K., Jhung, M. . . . Fry, A. (2011). A survey of emergency department 2009 pandemic influenza A (H1N1) surge preparedness: Atlanta, Georgia, July - October 2009. Clinical Infectious Diseases, 51(s1), s117-s182. doi: 10.1093/cid/ciq035

Suhonen, R., Stolt, M., Habermann, M., Hjaltadottir, I., Vryonides, S., Tonnessen, S. . . Scott, P. A. (2018). Ethical elements in priority setting in nursing care: A scoping review. International Journal of Nursing Studies, 88, 25-42. doi: 10.1016/j.ijnurstu.2018.08.006

Sullivan, S. J., Jacobsen, R. \& Poland, G. A. (2009). Mandating influenza vaccination for healthcare workers. Expert Review of Vaccines, 8(11), 1469-1474. doi: 10.1586/erv.09.118

Statistics New Zealand. (2019). Estimated population of NZ. Retrieved from https://www.stats.govt.nz/indicators/population-of-nz 
The Immunisation Advisory Centre. (2019). Eligibility criteria. Retrieved from https://www.influenza.org.nz/eligibility-criteria

Thompson, K. A., \& Bennett, A. M. (2017). Persistence of influenza on surfaces. Journal of Hospital Infection, 95(2), 194-199. doi: 10.1016/j.jhin.2016.12.003

Thorne, S. E. (2014). Applied interpretive approaches. In P. Leavy (Ed.), The Oxford handbook of qualitative research (pp. 99-115). Oxford, England: Oxford University Press.

Thorne, S., Kirkham, S. R., \& MacDonald-Emes, J. (1997). Interpretive description: A noncategorical qualitative alternative for developing nursing knowledge. Research in Nursing \& Health, 20(1), 169-177. Retrieved from https://onlinelibrary.wiley.com/journal/1098240x

Traianou, A. (2014). The centrality of ethics in qualitative research. In P. Leavy (Ed.), The Oxford handbook of qualitative research (pp. 62-77). Oxford, England: Oxford University Press.

Tucker, A. L., Heisler, W. S., \& Janisse, L. D. (2014). Designed for workarounds: A qualitative study of the causes of operational failures in hospitals. The Permanente Journal, 18(3), 33-41. doi: 10.7812/TPP/13-141

Turnberg, W., Daniell, W., Seixas, N., Simpson, T., Van Buren, J., Lipkin, E., \& Duchin, J. (2008). Appraisal of recommended repiratory infection control practices in primary care and emergency department settings. American Journal of Infection Control, 36(4), 268-275. doi: 10.1016/j.ajic.2007.08.004

Uyeki, T. M., Bernstein, H. H., Bradley, J. S., Englund, J. A., File, T. M., Fry, A. M. . . Pavia, A. T. (2019). Clinical practice guidelines by the infectious disease society of America: 2018 update on diagnosis, treatment, chemoprophylaxis, and 
institutional outbreak management of seasonal influenza. Clinical Infectious Diseases, 68(6), e1-e47. doi: 10.1093/cid/ciz044

Vaismoradi, M., Turunen, H., \& Bondas, T. (2013). Content analysis and thematic analysis: Implications for conducting a qualitative descriptive study. Nursing \& Health Sciences, 15(3), 398-405. doi: 10.1111/nhs.12048

Varia, M., Wilson, S., Sarwal, S., McGeer, A., Gournis, E., Galanis, E., \& Henry, B. (2003). Investigation of a nosocomial outbreak of severe acute respiratory syndrome (SARS) in Toronto, Canada. Canadian Medical Association Journal, 169(4), 285-292. Retrieved from http://www.cmaj.ca/content/169/4

Vertesi, L. (2004). Does the Canadian emergency department triage and acuity scale identify non-urgent patients who can be triaged away from the emergency department? Canadian Journal of Emergency Medicine, 6(5), 337-342. doi: $10.1017 / \mathrm{S} 1481803500009611$

Verweij, M. (2008). Moral principles for allocating scarce medical resources in an influenza pandemic. Journal of Bioethical Inquiry, 6(2), 159-169. doi: $10.1007 / \mathrm{s} 11673-009-9161-6$

Walter, S. R., Raban, M. Z., \& Westbrook, J. I. (2019). Visualising clinical work in the emergency department: Understanding interleaved patient management. Applied Ergonomics, 79, 45-53. doi: 10/1016/j.apergo.2019.04.005

Ward, P. R., Attwell, K., Meyer, S. B., Rokkas, P., \& Leask, J. (2017). Understanding the perceived logic of care by vaccine-hesitant and vaccine-refusing parents: $A$ qualitative study in Australia. PLOS ONE, 12(10), e0185955. doi: 10.1371/journal.pone.0185955

Water, T., Rasmussen, S., Neufeld, M., Gerrard, D., \& Ford, K. (2017). Nursing's duty of care: From legal obligation to moral commitment. Nursing Praxis in New 
Zealand, 33(3), 7-20. Retrieved from

https://www.questia.com/library/journal/1P4-2036387809/nursing-s-duty-ofcare-from-legal-obligation-to-moral

Weaver, K., \& Olson, J. K. (2006). Understandings paradigms used for nursing research. Journal of Advanced Nursing, 53(4), 459-469. doi: 10.1111/j.13652648-2006-03740.x

Westphal, J., Lancaster, R., \& Park, D. (2014). Work-arounds observed by fourth-year nursing students. Western Journal of Nursing Research, 36(8), 1002-18. doi: $10.1177 / 0193945913511707$

Whitehead, D. (2007). Common qualitative methods. In Z. Schneider, D. Whitehead, D. Elliott, G. LoBiondo-Wood, \& J. Haber (Eds.), Nursing and midwifery research: Methods and appraisal for evidence based practice. (3rd ed., pp. 105121). Sydney, Australia: Elsevier.

World Health Organisation. (2003). Consensus document on the epidemiology of severe acute respiratory syndrome (SARS). Retrieved from https://www.who.int/csr/sars/en/WHOconsensus.pdf

World Health Organisation. (2007). Ethical considerations in developing a public health response to pandemic influenza. Retrieved from https://www.who.int/csr/resources/publications/WHO_CDS_EPR_GIP_2007_2 c.pdf

World Health Organisation. (2010). What is a pandemic? Retrieved from https://www.who.int/csr/disease/swineflu/frequently_asked_questions/pande mic/en/

World Health Organisation. (2014). Influenza virus infections in humans (February 2014). Retrieved from 
http://www.who.int/influenza/human_animal_interface/virology_laboratories _and_vaccines/influenza_virus_infections_humans_feb14.pdf?ua=1

World Health Organisation. (2018). Influenza (avian and other zoonotic). Retrieved from https://www.who.int/en/news-room/fact-sheets/detail/influenza-(avianand-other-zoonotic)

World Health Organisation. (2019). Measles outbreak in the Pacific-situation report No 329 November 2019. Retrieved from https://www.who.int/westernpacific/emergencies/measles-outbreaks-in-thepacific

World Health Organisation. (2020). Statement on the meeting of the International Health Regulations (2005) Emergency Committee regarding the outbreak of novel coronavirus (2019-nCoV). Retrieved from https://www.who.int/newsroom/detail/23-01-2020-statement-on-the-meeting-of-the-internationalhealth-regulations-(2005)-emergency-committee-regarding-the-outbreak-ofnovel-coronavirus-(2019-ncov)

Zhou, J.-C., Pan, K.-H., Zhou, D.-Y., Zheng, S.-W., Zhu, J.-Q., Xu, Q.-P., \& Wang, C.-L. (2012). High hospital occupancy is associated with increased risk for patients boarding in the emergency department. American Journal of Medicine, 125(4), 416.e3. doi: 10.1016/j.amjmed.2011.07.030 


\section{APPENDICIES}

\section{Appendix 1: Locality approval}

Sent: Tuesday, 22 January 2019 3:19 PM

To: Jessica Buckley

Subject: RE: Masters research locality sign off

Hi Jess,

The study is approved for

and I will email you the signed form when available for your record. All the best and good lucke:

Kind Regards

\begin{tabular}{|l|l}
\hline & Research Office Manager \\
\hline Research Office &
\end{tabular}

Sent: Wednesday, 23 January 2019 3:13 PM

To: Jessica Buckley

Subject: RE: Masters Research locality sign off

Hi Jessica,

Your registration number is

( you can use that to advertise in

, good luck with the project and please email me the study results@

kind regards.
Research Office Manager 


\section{Appendix 2: Recruitment email}

Subject: Research invitation for a qualitative study exploring the impact of pandemic influenza on the ED

Dear ED nursing staff,

I am undertaking research to complete a thesis for my Masters of Nursing Science at Victoria University of Wellington. I would like to interview nurses working at

( ED who are triage trained and able to work in this area in your ED.

The research aims to answer the question 'what are the strategies and procesess emergency department nurses consider important to safely manage during an influenza pandemic?'. My aim is to identify ED nurses' concerns around the provision of nursing care during an influenza pandemic and their concerns around the safety of themselves, their families and ED patients. I hope to use this research to inform future pandemic planning in New Zealand EDs and identify strategies to improve nursing care during a pandemic.

This research is being carried out for academic purposes and not on behalf of your employer. Participation at all times is voluntary, and your decision to participate or not participate will not result in any adverse impact on your employment status or working conditions. Participation in this research would involve a face-to-face interview that will take approximately one hour and will be audio recorded. The interview will be at a time and place that is convenient to you as the participant. Any information you provide will be kept confidential and will be de-identified in the final thesis. You will be asked to sign a consent form before commencement of the interview and you may withdraw your consent to participate up to four weeks after the date of your interview.

If you are interested in participating in this research or would like further information, please contact:

Jessica Buckley

Thank you,

Jessica Buckley 


\section{RESEARCH STUDY ON THE IMPACT OF PANDEMIC INFLUENZA ON

I am conducting a research study to determine:

\section{What are the strategies and processes emergency} department nurses consider important to safely manage during an influenza pandemic?

Information will be gathered on this topic using face-to-face interviews during February 2019 Inclusion criteria:

- Currently working as a Registered nurse in an Emergency Department

- Work in the triage area of your Emergency Department

If you are interested in participating or would like further information, please contact:

Jessica Buckley

Email:

Masters of Nursing Science (Candidate)

Graduate School of Nursing, Midwifery and Health

Victoria University of Wellington

IB WHARE WĀNAVGA O TE OPOKO O TB IKA A MÃUI 5 :

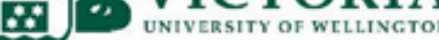

VUW Ethics Committee approval number: HEC 27012

\begin{tabular}{|c|c|c|c|c|c|c|c|c|}
\hline 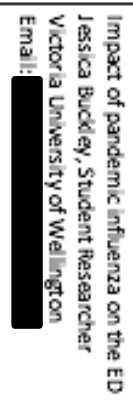 & 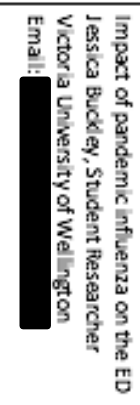 & 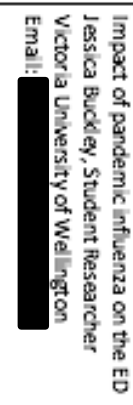 & 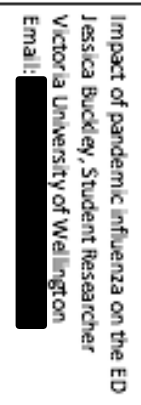 & 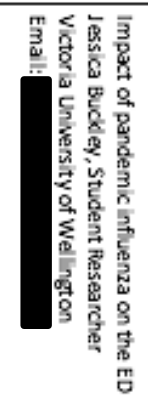 & 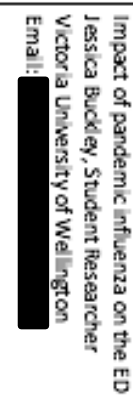 & 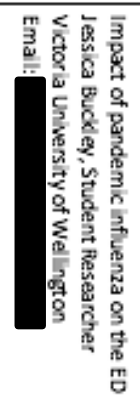 & 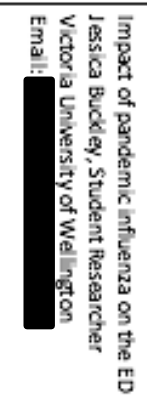 & 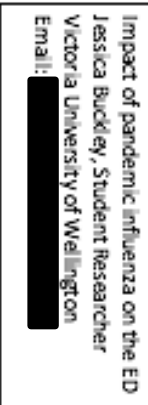 \\
\hline
\end{tabular}


Appendix 4: Participant demographic questionnaire

\section{Demographic Data}

\begin{tabular}{|l|l|}
\hline What is your age group? (please circle) \\
\hline $20-29$ & $50-59$ \\
\hline $30-39$ & $60+$ \\
\hline $40-49$ & \\
\hline
\end{tabular}

What gender do you identify with?

What is your ethnicity?

What is your role at your ED? (please circle)

\begin{tabular}{l|l} 
Registered Nurse & Senior Nurse
\end{tabular}

In what year did you first gain your nursing registration?

In what country did you first gain your nursing registration?

How many years have you worked in emergency nursing?

How many years have you worked in triage?

How many people live in your family home?

Are you a primary caregiver for any dependent children, or any family members with co-morbidities? (please circle)

Yes

No 


\section{Appendix 5: Semi-structured interview guide}

\section{Staff perceptions and concerns}

1. Tell me about how your Department manages seasonal influenza?

a. Prompts: Workload, staffing, resources

b. How does your ED manage surges of presentations?

2. How do you think an influenza pandemic might alter the way you deliver nursing care in your ED?

a. The Ministry of Health and DHBs require EDs to continue managing their normal workload as well as manage the surge of influenza patients during a pandemic. How do you see this working in your ED?

b. What strategies do you feel might be needed for your ED to manage the increased workload during a pandemic?

c. Another part of the Ministry of Health's pandemic plan involved keeping unwell people in the community to be cared for at home by family members. If you had a sick family member, how do you think this would affect your family?

\section{Presenteeism}

3. Tell me about how you decide when to call in sick to work?

a. What are your feelings when calling in sick?

b. How does workload in the ED impact on your decisions? 


\section{Infection control}

4. When do you decide to implement isolation precautions for a patient with influenza-like illness (ILI)?

a. Are there any factors that influence this decision? eg patient acuity, ED capacity

\section{$\underline{\text { Triage practices and principles }}$}

5. How do you manage patients that might have influenza at triage?

a. The DHB requires patients presenting with ILI to be given a mask and segregated from non-ILI patients within the ED waiting room. How is this requirement met in your ED?

b. A major part of the Ministry of Health and DHB plans for pandemic management requires ED nurses to triage lower-acuity ILI patients away from ED to GPs or community-based assessment centres. How do you feel about triaging patients away from ED during a pandemic?

\section{Occupational exposure to infectious disease}

6. There are many identified risks to caring for infectious patients. What are the specific risks in your ED when caring for influenza patients? 


\section{Appendix 6: Example of NVivo 12 content code (marked by inverted commas)}

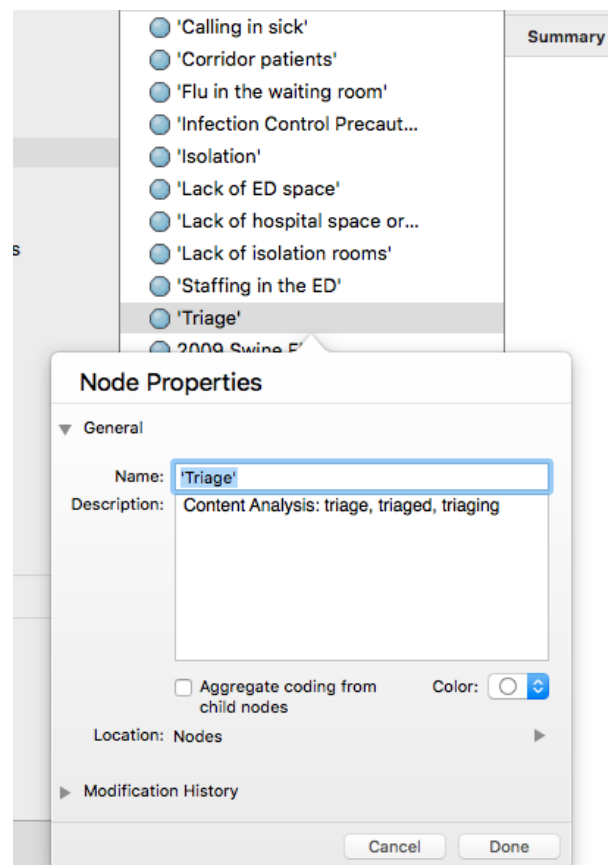

\section{Reference \\ FilesIIP1 Transcript de-identified} 15 references coded, $15.58 \%$ coverage

Reference $1: 0.51 \%$ coverage quite often the triage area and the waiting area has a number of patients waiting at any given time, or patients might be in corridors for example to be waiting for assessment or treatment or investigations, just because there's somebody else that needs to be seen in a private environment

Reference 2: $1.42 \%$ coverage

Yeah so I guess um-ah the way that our triage area works isn't its not isolated from the waiting room and I think obviously that's how most EDs are set up but it I think that realistically from the word go from the front where you need to be identifying patients that have got influenza possibly and knowing from like seasonal influenza when we've have had an influx of like patients that have got it - got influenza or suspected influenza you know they have to come in before they get a mask they have to actually present themselves to the front desk before and to talk to someone and not necessarily highlight what their symptoms are to the nursing staff that they could possibly have this infection and I guess it's...I don't think that we can cope even from the front door with stopping the transmission of um influenza

Reference 3: $1.25 \%$ coverage

same deal with like washing hands and things reduces how that is transmitted but it makes it difficult because there's not necessarily um great access to ensuring that hands are kept clean and that so I guess it's just possibly when we'd change where we might triage patients and um I don't think there is a process in place um that has been discussed widely as to what we would do at the-at the beginning you know if we did suspect something um but $I$ think we could most probablv attembt to redirect patients who have got suspected influenza 


\section{Appendix 7: Example of NVivo 12 thematic codes and raw data}

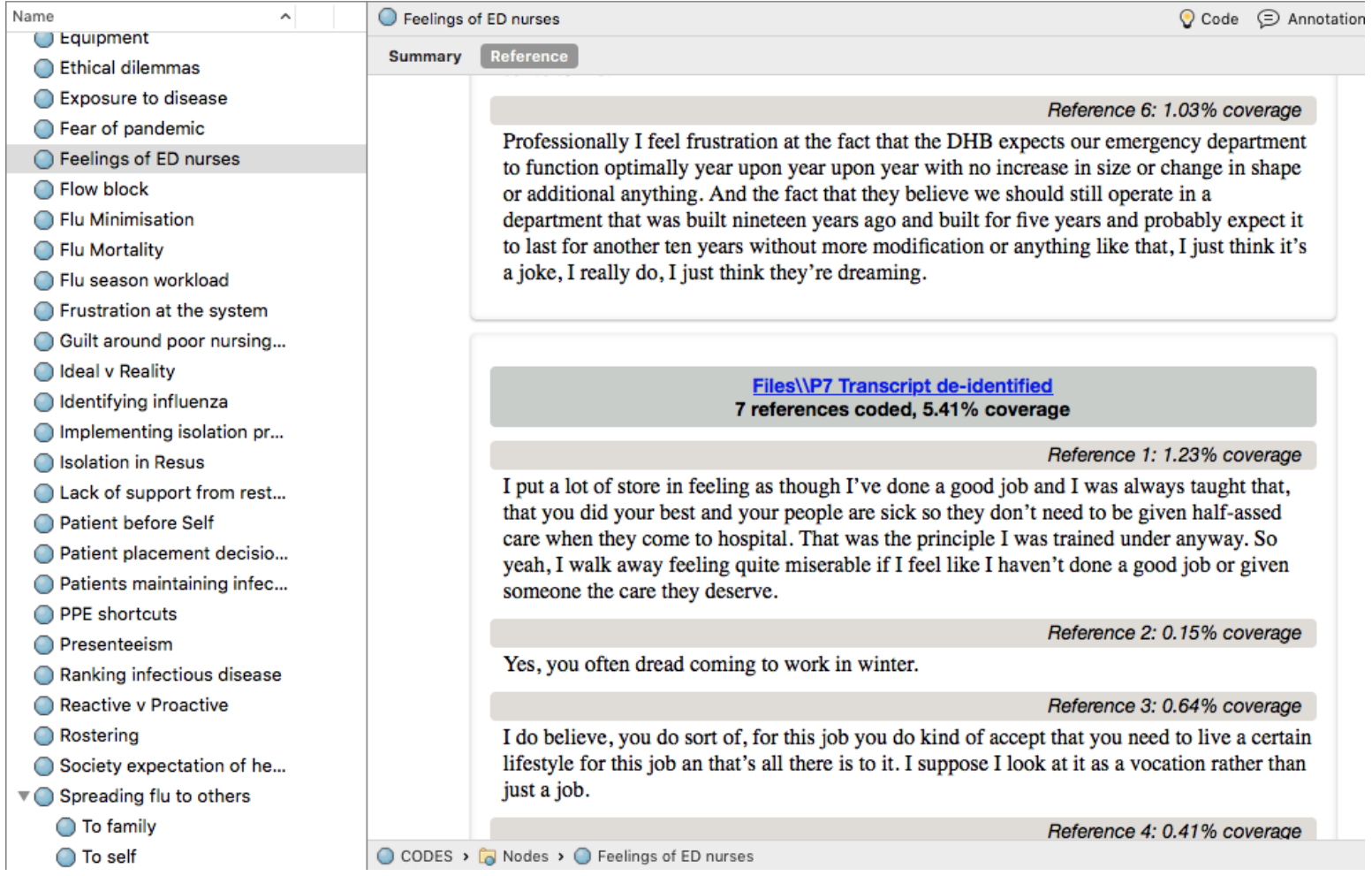




\begin{tabular}{l|c}
\hline TO & Jessica Colleen Mary Buckley \\
\hline FROM & Convenor, Human Ethics Committee \\
\hline
\end{tabular}

\begin{tabular}{l|l}
\hline DATE & 20 December 2018 \\
\hline PAGES & 1 \\
\hline
\end{tabular}

\begin{tabular}{l|l}
\hline SUBJECT & $\begin{array}{l}\text { Ethics Approval } \\
\text { Number: 0000027012 } \\
\text { Title: What are the strategies and processes emergency } \\
\text { department nurses consider important to safely manage during an } \\
\text { influenza pandemic? }\end{array}$ \\
\hline
\end{tabular}

Thank you for your application for ethical approval, which has now been considered by the Human Ethics Committee.

Your application has been approved from the above date and this approval is valid for three years. If your data collection is not completed by this date you should apply to the Human Ethics Committee for an extension to this approval.

Best wishes with the research.

Kind regards,

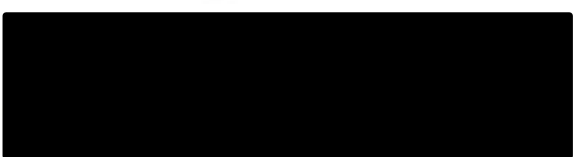

Convenor, Victoria University of Wellington Human Ethics Committee 


\section{Appendix 9: Participant information sheet}

TE WHARE WĀNAVGA O TE ŪPOKO O TE IKA A MĀUI 5 59

* * 4 university of wellington

\section{What are the strategies and processes emergency department nurses consider important to safely manage during an influenza pandemic?}

\section{INFORMATION SHEET FOR PARTICIPANTS}

You are invited to take part in this research. Please read this information before deciding whether or not to take part. If you decide to participate, thank you. If you decide not to participate, thank you for considering this request.

Who am I?

My name is Jessica Buckley and I am a Masters of Nursing Science student at Victoria University of Wellington. This research project is work towards the completion of my thesis.

What is the aim of the project?

This project aims to identify the concerns of ED nurses around the provision of nursing care during an influenza pandemic, and the impact a pandemic might have on the safety of ED patients, nursing staff and their families. This research aims to identify strategies to improve patient care in New Zealand EDs that could be used to inform future pandemic plans. This research has been approved by the Victoria University of Wellington Human Ethics Committee (HEC 27012).

How can you help?

You have been invited to participate because you are a triage-qualified ED nurse working in one of two public hospitals within the recruitment area. Having the ability to work within the triage area of your ED is important, as triage is an area that undergoes significant changes during a pandemic and this will be explored within this research. This research is being carried out for academic purposes and not on behalf of your employer. Participation at all times is voluntary, and your decision to participate or not participate will not result in any adverse impact on your employment status or working conditions.

If you agree to take part I will interview you at a location that is mutually agreed upon where you feel comfortable, at a time that is convenient for you. This could be your home or a private space at your public hospital campus. I will ask you questions about exposure to infectious disease in your workplace, how you feel an influenza pandemic would impact on your ED and the provision of nursing care, and infection control and triage processes in your workplace. I will also collect some demographic information at the commencement of the interview. The interview will take up to one hour.

I will audio record the interview with your permission and write it up later. I may also take notes during the interview to aid in analysis. You can choose to not answer any question or stop the interview at any time, without giving a reason. You can withdraw from the study by 
contacting me at any time up to four weeks after your interview. If you withdraw, the information you provided will be destroyed or returned to you.

\section{What will happen to the information you give?}

This research is confidential. This means that only I as the researcher will be aware of your identity, but the research data will be combined, and your identity will not be revealed in any reports, presentations, or public documentation. However, you should be aware that in small projects your identity might be obvious to others in your community. Confidentiality will be preserved except where you disclose something that causes me to be concerned about a risk of harm to yourself and/or others.

Only my supervisors and I will read the notes or transcript of the interview. My supervisor will only have access to de-identified data. The interview transcripts, summaries and any recordings will be kept securely and destroyed on 1/3/2024.

\section{What will the project produce?}

The information from my research will be used in the completion of my Masters research thesis. The research may also be published in an academic or professional journal. The results of this research may also be used to update existing DHB pandemic plans to include the strategies identified to improve nursing care in New Zealand EDs during a pandemic.

\section{If you accept this invitation, what are your rights as a research participant?}

You do not have to accept this invitation if you don't want to. If you do decide to participate, you have the right to:

- $\quad$ choose not to answer any question;

- $\quad$ ask for the recorder to be turned off at any time during the interview;

- $\quad$ withdraw from the study up to four weeks after the date of your interview;

- $\quad$ ask any questions about the study at any time;

- $\quad$ receive, read over and comment on a copy of your interview transcript;

- $\quad$ be able to read any reports of this research by emailing the researcher to request a copy.

If you have any questions or problems, who can you contact?

If you have any questions, either now or in the future, please feel free to contact either:

Student:

Name: Jessica Buckley

Email

\section{Supervisor:}

Name: Dr Caz Hales

Role: Lecturer

School: Graduate School of Nursing, Midwifery and Health

\section{Email:}

\section{Human Ethics Committee information}

If you have any concerns about the ethical conduct of the research you may contact the Victoria University HEC Convenor:

Email

telephone

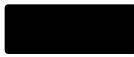




\section{Appendix 10: Consent form}

TE WHARE WÃNANGA O TE CPOKO O TE IKA A MÃUI

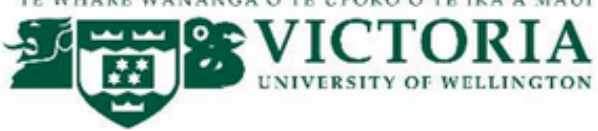

\section{What are the strategies and processes emergency department nurses consider important to safely manage during an influenza pandemic? \\ CONSENT TO INTERVIEW}

This consent form will be held for 5 years.

Researcher: Jessica Buckley, Graduate School of Nursing, Midwifery and Health, Victoria University of Wellington.

- I have read the Information Sheet and the project has been explained to me. My questions have been answered to my satisfaction. I understand that I can ask further questions at any time.

- I agree to take part in an audio recorded interview.

I understand that:

- I may withdraw from this study at any point up to four weeks after the date of my interview, and any information that I have provided will be returned to me or destroyed.

- The identifiable information I have provided will be destroyed on March $1^{\text {st }}, 2024$.

- Any information I provide will be kept confidential to the researcher and the supervisor.

- I understand that the results will be used for a Master of Nursing Science thesis and may be published in an academic or professional journal and presented to conferences.

- My name will not be used in reports, nor will any information that would identify me.

- I would like a copy of the transcript of my interview. Yes $\square$ No

- I would like to receive a copy of the final report and have added my email Yes $\square$ No address below.

Signature of participant:

Name of participant:

Date:

Contact email: 